\title{
EXPLICIT FORMULAS FOR GJMS-OPERATORS AND Q-CURVATURES
}

\author{
ANDREAS JUHL
}

\begin{abstract}
We describe GJMS-operators as linear combinations of compositions of natural second-order differential operators. These are defined in terms of PoincaréEinstein metrics and renormalized volume coefficients. As special cases, we derive explicit formulas for conformally covariant third and fourth powers of the Laplacian. Moreover, we prove related formulas for all Branson's $Q$-curvatures. The results settle and refine conjectural statements in earlier works. The proofs rest on the theory of residue families introduced in J09a.
\end{abstract}

November 9, 2018

\section{Contents}

1. Introduction and formulation of the main results

2. The inversion formula

3. Residue families

4. Residue families in terms of GJMS-operators

4.1. The pure $P$-terms

4.2. The pure $\bar{P}$-terms

4.3. The mixed terms

5. Factorization relations for $D_{2 N}(\lambda)$

6. The restriction property and the commutator relations 32

7. The principal part of $\mathcal{M}_{2 N}$

7.1. Description of the principal part

7.2. Proof of Theorem 7.1

8. The zeroth-order term of $\mathcal{M}_{2 N}$

8.1. General metrics 45

8.2. Conformally flat metrics 53

9. The explicit formula for $Q$-curvature $\quad 59$

10. Some low-order cases 63

10.1. The conformal cube $P_{6}$ of the Laplacian 63

10.2. The conformal fourth power $P_{8}$ of the Laplacian 67

11. Further results and comments 68

11.1. General signature 68

11.2. Einstein metrics 68

2010 Mathematics Subject Classification. Primary 05A19 35J30 53A30 53B20; Secondary 35 Q76 53A55 53C25 58J50.

${ }^{0}$ The work was supported by grant $621-2003-5240$ of the Swedish Research Council VR and SFB 647 "Space-Time-Matter" at Humboldt-University Berlin. 
11.3. Singular cases

11.4. More universal relations for GJMS-operators

11.5. The structure of the polynomials $\pi_{2 N}(\lambda)$

11.6. $Q_{2 N}^{\text {res }}(\lambda)$ and the universal recursive formula for $Q_{2 N}$

11.7. Contributions with many derivatives

11.8. The restriction property revisited

11.9. $\mathcal{M}_{2 N}$ and $\overline{\mathcal{M}}_{2 N}$ for round spheres

12. Appendix

12.1. The operators $\mathcal{M}_{2 N}$ for $N \leq 5$

12.2. The restriction property of $g_{(4)}$

12.3. The symmetry relation $g_{(4,2)}=g_{(2,4)}$

12.4. The coefficient $g_{(4,4)}$

12.5. Some further consequences of Theorem 7.2

References

\section{INTRODUCTION AND FORMULATION OF THE MAIN RESULTS}

In the present work, we discuss the structure of certain geometric differential operators of high order on Riemannian manifolds $M$ which are known as GJMS-operators. These operators are high-order generalizations of the Yamabe-operator]

$$
P_{2}(g) \stackrel{\text { def }}{=} \Delta_{g}-\left(\frac{n}{2}-1\right) \mathrm{J}_{g}, \quad \mathrm{~J}_{g}=\frac{\operatorname{scal}(g)}{2(n-1)} .
$$

All GJMS-operators $P_{2 N}(g)$ are natural differential operators of the form

$$
\Delta_{g}^{N}+\text { lower-order terms }
$$

which are conformally covariant in the sense that

$$
e^{\left(\frac{n}{2}+N\right) \varphi} P_{2 N}\left(e^{2 \varphi} g\right)(u)=P_{2 N}(g)\left(e^{\left(\frac{n}{2}-N\right) \varphi} u\right), u \in C^{\infty}(M)
$$

for all $\varphi \in C^{\infty}(M)$. The operators $P_{2 N}(g)$ were constructed by Graham, Jenne, Mason and Sparling in the seminal work GJMS92. They are induced, in a certain sense, by the powers of the Laplacian of the Fefferman-Graham ambient metric [FG07] on a space of two higher dimensions.

Operators which satisfy the conditions (1.2) and (1.3) are often referred to as conformally covariant powers of the Laplacian. Since these two conditions do not uniquely determine such operators, the GJMS-operators are to be considered as specific constructions of conformally covariant powers of the Laplacian.

The second operator in the sequence $P_{2}, P_{4}, P_{6}, \ldots$ of GJMS-operators is known as the Paneitz-operator $\mathrm{P} 08$. It is given by

$$
P_{4}=\Delta^{2}+\delta((n-2) \mathrm{J}-4 \mathrm{P}) d+\left(\frac{n}{2}-2\right)\left(\frac{n}{2} \mathrm{~J}^{2}-2|\mathrm{P}|^{2}-\Delta \mathrm{J}\right),
$$

where $\mathrm{P}$ denotes the Schouten tensor, i.e., $(n-2) \mathrm{P}=$ Ric $-J g$. In (1.4), the tensor $\mathrm{P}$ is regarded as an endomorphism on one-forms using $g$ and $|\mathrm{P}|^{2}=\mathrm{P}_{i j} \mathrm{P}^{i j}$.

\footnotetext{
${ }^{1}$ Here and throughout we use the convention that $-\Delta=\delta d$ is non-negative.
} 
On manifolds of odd dimension $n$, there are GJMS-operators $P_{2 N}$ of any order $2 N \geq 2$. But on manifolds of even dimension $n$, the construction in GJMS92 is obstructed at order $n$, i.e., does not give a conformally covariant power of Laplacian of order $>n$ for general metrics. In fact, by G92 (for $n=4$ and $N=3$ ) and GoH04 (in general) no such operator exists for general metrics. However, for locally conformally flat metrics and conformally Einstein metrics, the construction in GJMS92 yields conformally covariant powers of the Laplacian of any even order. In particular, we have an infinite sequence of such operators for all round spheres $\mathbb{S}^{n}$.

The GJMS-operators play a central role in conformal differential geometry and geometric analysis. More information on these aspects can be found in the recent monographs [DGH08, [J09a], [BJ10] and the references therein.

The complexity of lower-order terms in (1.2) grows exponentially with $N$. Therefore, finding an explicit description of these terms seems to be an almost impossible task. An additional problem is that the lower-order terms can be written in many different ways. Nevertheless, in the present work we shall solve this problem by providing a description of the GJMS-operators (and of the related Branson's $Q$-curvatures) which is both almost explicit and esthetically appealing. The main insight is that the enormous complexity of these operators can be described in terms of a sequence of natural second-order operators.

We continue with the formulation of the main results.

For this purpose, we need to introduce some more notation.

First, we shall use the following combinatorial conventions. A sequence $I=$ $\left(I_{1}, \ldots, I_{r}\right)$ of integers $I_{j} \geq 1$ will be regarded as a composition of the natural number $|I|=I_{1}+I_{2}+\cdots+I_{r}$. In other words, compositions are partitions in which the order of the summands is considered. $|I|$ will be called the size of $I$. To any composition $I=\left(I_{1}, \ldots, I_{r}\right)$, we associate the numbers

$$
m_{I}=-(-1)^{r}|I| !(|I|-1) ! \prod_{j=1}^{r} \frac{1}{I_{j} !\left(I_{j}-1\right) !} \prod_{j=1}^{r-1} \frac{1}{I_{j}+I_{j+1}}
$$

and

$$
n_{I}=\prod_{j=1}^{r}\left(\begin{array}{c}
\sum_{k \leq j} I_{k}-1 \\
I_{j}-1
\end{array}\right)\left(\begin{array}{c}
\sum_{k \geq j} I_{k}-1 \\
I_{j}-1
\end{array}\right) .
$$

Note that $m_{(N)}=n_{(N)}=1$ for all $N \geq 1$ and $n_{(1, \ldots, 1)}=1$. Moreover, for any composition $I$, we define the operator

$$
P_{2 I}=P_{2 I_{1}} \circ \cdots \circ P_{2 I_{r}}
$$

Then we set

$$
\mathcal{M}_{2 N}=\sum_{|I|=N} m_{I} P_{2 I}=P_{2 N}+\text { compositions of lower-order GJMS-operators. }
$$

These sums contain $2^{N-1}$ terms. Similarly, we define

$$
\mathcal{M}_{2 I}=\mathcal{M}_{2 I_{1}} \circ \cdots \circ \mathcal{M}_{2 I_{r}} \text {. }
$$

Second, the present theory rests on the notion of Poincaré-Einstein metrics

$$
g_{+}=r^{-2}\left(d r^{2}+g_{r}\right), g_{0}=g
$$


in the sense of Fefferman and Graham (see [FG85] and [FG07]). Here $g_{r}$ is a family of metrics on $M$ so that $g_{+}$is Einstein on the space $X=M \times(0, \varepsilon)$ in the sense that

$$
\operatorname{Ric}\left(g_{+}\right)+n g_{+}=0 \text {. }
$$

In particular, $g_{+}$has negative scalar curvature $-n(n+1)$. This concept will be recalled in Section 3. The volume form of a Poincaré-Einstein metric $g_{+}$relative to $g$ takes the form

$$
\operatorname{dvol}\left(g_{+}\right)=r^{-n-1} v(r) \operatorname{dvol}(g) d r
$$

with

$$
v(r)=1+v_{2} r^{2}+v_{4} r^{4}+\cdots+v_{n} r^{n}+\cdots ;
$$

here $v_{n}=0$ if $n$ is odd. In odd dimensions $n$, all coefficients $v_{2}, v_{4}, \ldots$ are scalarvalued curvature invariants of $g$. Similarly, in even dimensions $n$, the coefficients $v_{2}, \ldots, v_{n}$ are uniquely determined by $g$. The functionals $g \mapsto v_{2 j}(g)$ are known as renormalized volume coefficients (see [G00], G09]) or holographic coefficients (see [J09a]). In even dimensions $n$, the quantity $v_{n}$ is the (infinitesimal) conformal anomaly of the renormalized volume of $g_{+}$(see [G00]) 2 Now we set

$$
w(r)=\sqrt{v(r)}=1+w_{2} r^{2}+w_{4} r^{4}+\cdots+w_{n} r^{n}+\cdots
$$

with $w_{n}=0$ for odd $n$. The coefficients $w_{2}, w_{4}, w_{6}, \ldots$ are polynomials in $v_{2}, v_{4}, v_{6}, \ldots$ In particular,

$$
2 w_{2}=v_{2}, \quad 8 w_{4}=4 v_{4}-v_{2}^{2} \quad \text { and } \quad 16 w_{6}=8 v_{6}-4 v_{4} v_{2}+v_{2}^{3} .
$$

Finally, we introduce the generating function

$$
\mathcal{H}(r) \stackrel{\text { def }}{=} \sum_{N \geq 1} \mathcal{M}_{2 N} \frac{1}{(N-1) !^{2}}\left(\frac{r^{2}}{4}\right)^{N-1}
$$

of the sequence $\mathcal{M}_{2}, \mathcal{M}_{4}, \ldots$ Here we use the following convention. For even $n$, the sum in (1.7) is defined to run only up to $r^{n-2}$. For odd $n$, GJMS-operators are welldefined for any order $2 N \geq 2$, and the sum in (1.7) is regarded as a formal infinite power series.

The first main result gives formulas for all GJMS-operators.

Theorem 1.1. All GJMS-operators on Riemannian manifolds $(M, g)$ of dimension $n \geq 3$ can be written in the form

$$
P_{2 N}=\sum_{|I|=N} n_{I} \mathcal{M}_{2 I}=\mathcal{M}_{2}^{N}+\text { compositions with fewer factors. }
$$

Moreover, all operators $\mathcal{M}_{2 N}(g)$ are (at most) second-order and are given by the formula

$$
\mathcal{H}(g)(r)=-\delta\left(g_{r}^{-1} d\right)-r^{-2}\left(\Delta_{g_{+}}(\log w)-|d \log w|_{g_{+}}^{2}\right) .
$$

Here $\delta$ is defined with respect to $g$, and we regard $g_{r}$ as an endomorphism on one-forms using $g$. Finally, $w$ is regarded as a function on $X$.

\footnotetext{
${ }^{2}$ Sometimes $v_{n}$ is called the holographic anomaly.
} 
The relation (1.9) is to be understood as an identity of formal power series asserting that the coefficients on both sides coincide.

Theorem 1.1 states that all GJMS-operators of $(M, g)$ are generated by the Taylor coefficients of the one-parameter family $r \mapsto \mathcal{D}(g)(r)$ of second-order differential operators on $M$ defined by the right-hand side of (1.9). In view of $\mathcal{D}(g)(0)=P_{2}$, this family can be viewed as a (geometrically defined) perturbation of the Yamabe operator 3

The formulation of Theorem 1.1 does not require explicit formulas for the PoincaréEinstein metric $g_{+}$, i.e., does not require to solve the Einstein equation (1.6). But, of course, any such knowledge makes (1.9) more explicit.

In particular, for a locally conformally flat metric $g$, we have

$$
\left(g_{r}\right)_{i j}=g_{i j}-r^{2} \mathrm{P}_{i j}+\frac{r^{4}}{4} \mathrm{P}_{i k} \mathrm{P}_{j}^{k}
$$

and the construction of GJMS-operators is not obstructed for orders exceeding the dimension of the underlying manifold [FG07]. Since $g_{r}$ takes the form $\left(1-r^{2} / 2 \mathrm{P}\right)^{2}$, when regarded as a linear operator on one-forms using $g$, we find

$$
g_{r}^{-1}=\left(1-r^{2} / 2 \mathrm{P}\right)^{-2}=\sum_{N \geq 1} N \mathrm{P}^{N-1}\left(\frac{r^{2}}{2}\right)^{N-1}
$$

and

$$
w(r)=\sqrt{\operatorname{det}\left(1-r^{2} / 2 \mathrm{P}\right)} .
$$

In this special case, the assertions in Theorem 1.1 extend to all orders. (1.9) shows that the operator $\mathcal{M}_{2 N}$ has the principal part

$$
-(N-1) ! N ! 2^{N-1} \delta\left(\mathrm{P}^{N-1} d\right)
$$

and that the zeroth-order term of $\mathcal{M}_{2 N}$ equals the sum

$$
-(N-1) !^{2} 2^{N-1}\left(\left(\frac{n}{2}-N\right) \operatorname{tr}\left(\mathrm{P}^{N}\right)+\frac{1}{2} \sum_{a=1}^{N-1} \operatorname{tr}\left(\mathrm{P}^{a}\right) \operatorname{tr}\left(\mathrm{P}^{N-a}\right)\right)
$$

(modulo contributions involving derivatives). In combination with (1.8), these results yield explicit formulas for all GJMS-operators in terms of $g$ and P. In the special case of the round sphere $\mathbb{S}^{n}$, we have

$$
g_{r}^{-1}=\left(1-r^{2} / 4\right)^{-2} \text { id } \text { and } w(r)=\left(1-r^{2} / 4\right)^{\frac{n}{2}},
$$

and (1.9) simplifies to

$$
\mathcal{H}(r)=\left(1-r^{2} / 4\right)^{-2}\left(\Delta-\frac{n}{2}\left(\frac{n}{2}-1\right)\right)=\left(\sum_{N \geq 1} N\left(r^{2} / 4\right)^{N-1}\right) P_{2} .
$$

In other words, the second part of Theorem 1.1 states that

$$
\mathcal{M}_{2 N}=N !(N-1) ! P_{2} \text { for } N \geq 1 .
$$

These summation formulas were proved in [J09b] by using the product formula (11.1) for the GJMS-operators of $\mathbb{S}^{n}$ (see [B95], G07]).

\footnotetext{
${ }^{3}$ It is also tempting to consider it as an operator $C^{\infty}(M) \rightarrow C^{\infty}(M \times[0, \varepsilon))$.
} 
For general metrics, the Taylor coefficients of $w(r)$ can be expressed in terms of renormalized volume coefficients which, in turn, can be written in terms of the Schouten tensor $\mathrm{P}$ and Graham's extended obstruction tensors $\Omega^{(k)}$ (see Section 2 of G09]). Note that $\Omega^{(1)}=\mathcal{B} /(4-n)$, where $\mathcal{B}$ is the Bach tensor. In low-order cases, the description of $P_{2 N}$ in Theorem 1.1 can be made more explicit. The corresponding results for $P_{6}$ and $P_{8}$ are stated in Section 10 .

Theorem 1.1 describes any GJMS-operator in terms of second-order operators. Alternatively, the second part of Theorem 1.1 can be rephrased by stating that any GJMS-operator $P_{2 N}$ is the sum of the linear combination

$$
-\sum_{|I|=N, I \neq(N)} m_{I} P_{2 I}
$$

of compositions of lower-order GJMS-operators and the second-order operator given by the coefficient of

$$
\frac{1}{(N-1) !^{2}}\left(\frac{r^{2}}{4}\right)^{N-1}
$$

in the Taylor series of $\mathcal{D}(g)(r)$. We shall refer to such identities as to recursive formulas for GJMS-operators.

Finally, we emphasize the following fact. The definition (1.5) shows that, for any $N \geq 1$, the operators $\mathcal{M}_{2 N}$ are of order $\leq 2 N$. The result that the operators $\mathcal{M}_{2 N}$ are actually only of second order can be regarded as a remarkable cancellation effect.

The second main result of the present paper is a related universal formula for all Branson's $Q$-curvatures. We recall that, for even $n$ and $2 N<n$, the GJMS-operator $P_{2 N}$ gives rise to a scalar curvature quantity $Q_{2 N}$ via the formula

$$
P_{2 N}(g)(1)=(-1)^{N}\left(\frac{n}{2}-N\right) Q_{2 N}(g) \text {. }
$$

The critical $Q$-curvatures $Q_{n}$ (for even $n$ ) can be defined through the non-critical $Q$-curvatures $Q_{2 N}(2 N<n)$ by a limiting process. For odd $n$, the quantities $Q_{2 N}$ are defined for all $N \geq 1$ by (1.12). For more information and a discussion of the significance of $Q$-curvature we refer to [B95], B05], BG08], DGH08, J09a] and [BJ10].

Theorem 1.2. For even $n$ and $2 N \leq n$ and for odd $n$ and all $N \geq 1$,

$$
(-1)^{N} Q_{2 N}=\sum_{|I|+a=N} n_{(I, a)} a !(a-1) ! 2^{2 a} \mathcal{M}_{2 I}\left(w_{2 a}\right) .
$$

Here we use the convention that the sum on the right-hand side of (1.13) contains a contribution by $w_{2 N}$ (for the trivial composition $I$ ). Note that the number of terms in that sum is $2^{N-1}$, i.e., it grows exponentially with $N$.

The formula (1.13) follows by combining the first part of Theorem 1.1 with structural results for the zeroth-order terms $\mathcal{M}_{2 N}(1)$ of the operators $\mathcal{M}_{2 N}$ (Theorem 8.2 and Theorem 8.3). In turn, the proofs of these results utilize the universal recursive formula

$$
\sum_{|I|+a=N} m_{(I, a)}(-1)^{a} P_{2 I}\left(Q_{2 a}\right)=N !(N-1) ! 2^{2 N} w_{2 N}
$$


for $Q$-curvature proved in [J10b].

For locally conformally flat metrics, Theorem 1.2 extends to all $Q$-curvatures $Q_{2 N}$.

Of course, Theorem 1.2 shows its full power only when it is combined with the explicit formula for the operators $\mathcal{M}_{2 N}$ in the second part of Theorem 1.1. It is interesting to compare (1.13) and (1.14). First of all, we note that both formulas are universal in the dimension $n$. Next, the formula (1.13) actually should be regarded as a resolution of the recursive structure of $Q$-curvatures described in (1.14). If we split off the contributions of the trivial composition, then (1.13) and (1.14) take the respective forms

$$
(-1)^{N} Q_{2 N}=\sum_{\substack{|I|+a=N \\ a \leq N-1}} n_{(I, a)} a !(a-1) ! 2^{2 a} \mathcal{M}_{2 I}\left(w_{2 a}\right)+N !(N-1) ! 2^{2 N} w_{2 N}
$$

and

$$
(-1)^{N} Q_{2 N}+\sum_{\substack{|I|+a=N \\ a \leq N-1}} m_{(I, a)}(-1)^{a} P_{2 I}\left(Q_{2 a}\right)=N !(N-1) ! 2^{2 N} w_{2 N} .
$$

In both formulas, we have collected terms of the same nature on the same sides. The latter identities clearly show that (1.13) and (1.14) express the differences

$$
(-1)^{N} Q_{2 N}-N !(N-1) ! 2^{2 N} w_{2 N}
$$

in two conceptually different ways 4 For more details concerning the low-order cases $N \leq 4$ we refer to Section 9 .

Note that for $a=1$ and the composition $I=(1, \ldots, 1)$ of size $N-1$ the right-hand side of (1.13) contains the contribution $-\Delta^{N-1} \mathrm{~J}$. This reproduces the well-known fact [B95] that the contribution to $Q_{2 N}$ which contains the maximal number of derivatives is $(-1)^{N-1} \Delta^{N-1} \mathrm{~J}$.

Finally, the relations (1.13) and (1.14) should be compared with the holographic formulas of [GJ07] and [J10a] for the differences

$$
(-1)^{N} Q_{2 N}-N !(N-1) ! 2^{2 N-1} v_{2 N} .
$$

In the critical case $2 N=n$, the holographic formula shows that the latter difference is an exact divergence. Hence for closed manifolds the global conformal invariants

$$
\int_{M} Q_{n} d v o l \text { and } \int_{M} v_{n} d v o l
$$

are proportional GZ03. This fact plays a basic role, for instance, in GH05. A major difference between the holographic formulas and the formulas (1.13), (1.14) is that the metric variations of the Laplacian which contribute to the former relations (through the asymptotic expansions of eigenfunctions) do not appear in the latter ones.

Theorem 1.1 and Theorem 1.2 yield explicit formulas for GJMS-operators and $Q$-curvatures in the sense that both objects are described in terms of (the Taylor coefficients of) associated Poincaré-Einstein metrics. In order to derive fully explicit expressions for GJMS-operators and $Q$-curvatures in terms of the metrics, it suffices

\footnotetext{
${ }^{4}$ These formulas simplify even a bit further if the coefficients $w_{2 N}$ are defined as the coefficients of $w(r)$ as a power series in $r / 2$. However, we prefer to use the same notation as in [J10b].
} 
to combine them with corresponding formulas for the Poincaré-Einstein metrics. The natural building blocks of such formulas are Graham's extended obstruction tensors G09.

Finally, we note that in GoP03 Gover and Peterson developed an alternative approach to GJMS-operators and $Q$-curvatures using tractor calculus. A remarkable feature of their method is that it yields explicit formulas in terms of the metric without solving the Einstein equation (1.6).

The structure of the paper is as follows. Section 2 contains the proof of the first assertion of Theorem [1.1. In the following three sections we recall the notion of residue families introduced in [J09a], prove their factorization relations in full generality (Theorem 3.1 and Theorem 3.2), and derive from these a representation formula for these families in terms of GJMS-operators (Theorem 4.1). It is this representation formula which explains the role of the operators $\mathcal{M}_{2 N}$. Only by reasons of degree, Theorem 4.1 implies two basic properties of the sums $\mathcal{M}_{2 N}$ which we refer to as the restriction property (Theorem 6.3) and the commutator relations (Theorem 6.4). The commutator relations are the main device to prove that the operators $\mathcal{M}_{2 N}$ are second-order and to derive the formula for their principal parts (Theorem 7.1). An important tool in the proof of this formula is Theorem [7.2. The latter result might be also of independent interest. In Section 8 , we determine the the zeroth-order terms of the operators $\mathcal{M}_{2 N}$. We find (Theorem 8.2 and Theorem 8.3) that these curvature quantities satisfy recursive relations. These relations are closely related to the recursive structure of $Q$-curvatures. The results lead to the proof of the second part of Theorem 1.1, when combined with the description of the principal part of $\mathcal{M}_{2 N}$ in Theorem 7.1. Since these arguments do not suffice to cover also the supercritical GJMS-operators for locally conformally flat metrics, Theorem 8.4 provides an alternative proof of Theorem 8.3 (for such metrics) which does extend to the supercritical cases. It does not refer to $Q$-curvature. Instead, it rests on an evaluation of the restriction property and the commutator relations, i.e., is similar in spirit to the proof of the formula for the leading terms of $\mathcal{M}_{2 N}$. Finally, Theorem 8.5 derives the contributions described in (1.11). Section 9 is devoted to the proof of Theorem [1.2. As illustrations of the general theory, and for the convenience of the reader interested mainly in low-order special cases, we make explicit in Section 10, the results in the special cases of the conformally covariant third and fourth power of the Laplacian. In Section 11, we comment on a number of scattered issues, sketch some further results and point out a few open problems. The paper finishes with an appendix which contains direct proofs of some low-order special cases of Theorem 7.2 and a description of a resulting algorithm which reproduces Graham's formulas for Poincaré-Einstein metrics in terms of the Schouten tensor and the extended obstruction tensors.

Some early steps of the work were influenced by discussions with Vladimir Souček and Petr Somberg during a visit at Charles University (Prague) in spring 2010. Parts of the results were presented at the workshop on Parabolic Geometries and Related Topics of the University of Tokyo in November 2010, at Toronto University in April 2011 and at the workshop Cartan Connections, Geometry of Homogeneous Spaces, and Dynamics at the Erwin Schrödinger Institute (ESI) in Vienna in July 2011. We gratefully acknowledge the support by these institutions. The proof of the inversion 
formula in Section 2 is due to Christian Krattenthaler (Vienna). It is a pleasure to thank him for allowing to include his proof here. Many stages of the work involved computer experiments. In particular, we derived structural insight from numerical data and used the computer to test predictions. The corresponding calculations were performed using Mathematica with the NCAlgebra-packag 5 . We thank Carten Falk (Humboldt Universität, Berlin) for his invaluable efforts to create the numerous programs. Finally, we are grateful to Robin Graham for a discussion of subtle questions concerning super-critical GJMS-operators.

\section{THE INVERSION FORMULA}

We first recall some basic definitions from Section 1. For any composition $I=$ $\left(I_{1}, \ldots, I_{r}\right)$, we set

$$
m_{I}=-(-1)^{r}|I| !(|I|-1) ! \prod_{j=1}^{r} \frac{1}{I_{j} !\left(I_{j}-1\right) !} \prod_{j=1}^{r-1} \frac{1}{I_{j}+I_{j+1}}
$$

and

$$
n_{I}=\prod_{j=1}^{r}\left(\begin{array}{c}
\sum_{k \leq j} I_{k}-1 \\
I_{j}-1
\end{array}\right)\left(\begin{array}{c}
\sum_{k \geq j} I_{k}-1 \\
I_{j}-1
\end{array}\right) \in \mathbb{N} .
$$

Then $m_{(N)}=n_{(N)}=1$ for all $N \geq 1$ and

$$
m_{\left(I_{1}, I_{2}\right)}=-\left(\begin{array}{c}
N-1 \\
I_{1}
\end{array}\right)\left(\begin{array}{c}
N-1 \\
I_{2}
\end{array}\right)=-n_{\left(I_{1}, I_{2}\right)}
$$

for all compositions $I=\left(I_{1}, I_{2}\right)$ of size $N \geq 2$. In the following, we shall also work with an alternative expression for $n_{I}$ with reduced fractions. In fact, an easy calculation shows that

$$
n_{I}=(|I|-1) !^{2} \prod_{j=1}^{r} \frac{1}{\left(I_{j}-1\right) !^{2}} \prod_{j=1}^{r-1} \frac{1}{\left(\sum_{k=1}^{j} I_{k}\right)\left(\sum_{k=j+1}^{r} I_{k}\right)} .
$$

Note also that

$$
m_{I^{-1}}=m_{I} \quad \text { and } \quad n_{I^{-1}}=n_{I}
$$

where $I^{-1}=\left(I_{r}, \cdots, I_{1}\right)$ is the inverse composition of $I=\left(I_{1}, \cdots, I_{r}\right)$.

Moreover, we define

$$
P_{2 I}=P_{2 I_{1}} \circ \cdots \circ P_{2 I_{r}}
$$

and set

$$
\mathcal{M}_{2 N}=\sum_{|I|=N} m_{I} P_{2 I}
$$

The latter sum contains $2^{N-1}$ terms. Note that $\mathcal{M}_{2}=P_{2}$. Since all GJMS-operators are formally self-adjoint [GZ03], Eq. (2.4) implies that the operators $\mathcal{M}_{2 N}$ are formally self-adjoint, too. Finally, we set

$$
\mathcal{M}_{2 I}=\mathcal{M}_{2 I_{1}} \circ \cdots \circ \mathcal{M}_{2 I_{r}} .
$$

By (2.4), we also have $\mathcal{M}_{2 I}=\mathcal{M}_{2 I}^{*}$.

\footnotetext{
${ }^{5}$ http://math.ucsd.edu/ ncalg/
} 
The following result states in which sense the operators $\mathcal{M}_{2 N}$ can be regarded as the building blocks of the GJMS-operators.

Theorem 2.1. (Inversion Formula) For $N \geq 1$, we have

$$
P_{2 N}=\sum_{|I|=N} n_{I} \mathcal{M}_{2 I}
$$

We refer to Theorem 2.1 as an inversion formula since it can be regarded as a description of the inverse of the transformation

$$
\left\{P_{2}, P_{4}, P_{6}, \cdots\right\} \rightarrow\left\{\mathcal{M}_{2}, \mathcal{M}_{4}, \mathcal{M}_{6}, \cdots\right\}
$$

defined by (2.6). The inversion formula is an algebraic fact which relates two sets of operators. Therefore, it is justified to omit here the assumptions which guarantee the existence of the GJMS-operators.

Note that the sums on the right-hand sides of (2.8) contain $2^{N-1}$ terms, respectively.

Note also that, in view of (2.3), the inversion formula can be written in the alternative form

$$
\frac{P_{2 N}}{(N-1) !^{2}}=\sum_{|I|=N} \frac{1}{q_{I}} \frac{\mathcal{M}_{2 I}}{\prod_{j=1}^{r}\left(I_{j}-1\right) !^{2}}
$$

with

$$
q_{I}=\prod_{j=1}^{r-1}\left(\sum_{k=1}^{j} I_{k}\right)\left(\sum_{k=j+1}^{r} I_{k}\right) .
$$

Proof. ([K10]) We substitute (2.6) in the right-hand side of (2.8) to obtain

$$
\sum_{|I|=N} n_{I} \sum_{\left|J_{1}\right|=I_{1}, \ldots,\left|J_{r}\right|=I_{r}}\left(\prod_{k=1}^{r} m_{J_{k}}\right) P_{2 J_{1}} \circ P_{2 J_{2}} \circ \cdots \circ P_{2 J_{r}} .
$$

Let $K=\left(K_{1}, K_{2}, \ldots, K_{s}\right)$ be a fixed composition of $N$. Then the coefficient of $P_{2 K}$ in (2.11) is given by

$$
\begin{aligned}
& \sum_{A \subseteq[s-1]} n_{\left(K_{1}+\cdots+K_{a_{1}}, K_{a_{1}+1}+\cdots+K_{a_{2}}, \ldots, K_{a_{\ell-1}+1}+\cdots+K_{a_{\ell}}, K_{a_{\ell}+1}+\cdots+K_{s}\right)} \\
& \cdot m_{\left(K_{1}, \ldots, K_{a_{1}}\right)} m_{\left(K_{a_{1}+1}, \ldots, K_{a_{2}}\right)} \cdots m_{\left(K_{a_{\ell-1}+1}, \ldots, K_{a_{\ell}}\right)} m_{\left(K_{a_{\ell}+1}, \ldots, K_{s}\right)} .
\end{aligned}
$$

The sum in (2.12) runs over all subsets $A=\left\{a_{1}, a_{2}, \ldots, a_{\ell}\right\} \subseteq\{1,2, \ldots, s-1\}$ (including the empty set) whereby we use the convention that $a_{1}<a_{2}<\cdots<a_{\ell}$. Using (2.1) and (2.3), after some simplification this expression becomes

$$
\begin{aligned}
& (-1)^{s+1}(N-1) !^{2} \prod_{j=1}^{s} \frac{1}{K_{j} !\left(K_{j}-1\right) !} \prod_{j=1}^{s-1} \frac{1}{\left(K_{j}+K_{j+1}\right)} \\
& \quad \times \sum_{A \subseteq[s-1]}(-1)^{|A|}\left(K_{1}+\cdots+K_{a_{1}}\right)\left(K_{a_{1}+1}+\cdots+K_{a_{2}}\right) \cdots\left(K_{a_{\ell}+1}+\cdots+K_{s}\right)
\end{aligned}
$$




$$
\cdot \frac{\prod_{a \in A}\left(K_{a}+K_{a+1}\right)}{\prod_{a \in A}\left(K_{1}+\cdots+K_{a}\right)\left(K_{a+1}+\cdots+K_{s}\right)},
$$

where $|A|$ stands for the cardinality of the set $A$, as usual. We have to show that the sum in (2.13) vanishes for $s>1$. That it equals 1 for $s=1$ is obvious. The following lemma implies our claim (by setting $X=Y=0$ ).

Lemma 2.1. For $s>1$, we have

$$
\begin{gathered}
\sum_{A \subseteq[s-1]}(-1)^{|A|}\left(K_{1}+\cdots+K_{a_{1}}\right)\left(K_{a_{1}+1}+\cdots+K_{a_{2}}\right) \cdots\left(K_{a_{\ell}+1}+\cdots+K_{s}+X\right) \\
\cdot \frac{\prod_{a \in A}\left(K_{a}+K_{a+1}+Y \cdot \chi(a=s-1)\right)}{\prod_{a \in A}\left(K_{1}+\cdots+K_{a}\right)\left(K_{a+1}+\cdots+K_{s}\right)} \\
=-\frac{X\left(K_{1}+\cdots+K_{s-1}\right)+Y\left(K_{s}+X\right)}{K_{2}+\cdots+K_{s}},
\end{gathered}
$$

where $\chi(\mathcal{S})=1$ if $\mathcal{S}$ is true and $\chi(\mathcal{S})=0$ otherwise.

Proof. We prove the claim by induction on $s$. For $s \geq 1$, let $f(s ; X, Y)$ denote the left-hand side of (2.14) . In particular, we have

$$
f(1 ; X, Y)=K_{1}+X \text {. }
$$

The claim (2.14) can be readily verified for $s=2$. Now we suppose that (2.14) has been proved up to $s$.

We consider $f(s+1 ; X, Y)$. We split the defining sum according to the largest element of the indexing set $A, t$ say, so that $A=A^{\prime} \cup\{t\}$. In this manner, we obtain

$$
\begin{gathered}
f(s+1 ; X, Y)=\left(K_{1}+\cdots+K_{s+1}+X\right) \\
-\sum_{t=1}^{s} \frac{\left(K_{t+1}+\cdots+K_{s+1}+X\right)\left(K_{t}+K_{t+1}+Y \cdot \chi(t=s)\right)}{\left(K_{1}+\cdots+K_{t}\right)\left(K_{t+1}+\cdots+K_{s+1}\right)} \\
\cdot \sum_{A^{\prime} \subseteq[t-1]}(-1)^{\left|A^{\prime}\right|}\left(K_{1}+\cdots+K_{a_{1}^{\prime}}\right) \cdots\left(K_{a_{\ell-1}^{\prime}+1}+\cdots+K_{t}\right) \\
\cdot \frac{\prod_{a \in A^{\prime}}\left(K_{a}+K_{a+1}\right)}{\prod_{a \in A^{\prime}}\left(K_{1}+\cdots+K_{a}\right)\left(K_{a+1}+\cdots+K_{s+1}\right)} .
\end{gathered}
$$

We may rewrite this as

$$
\begin{aligned}
f(s+1 ; X, Y)= & \left(K_{1}+\cdots+K_{s+1}+X\right) \\
-\sum_{t=1}^{s-1} \frac{\left(K_{t+1}+\cdots+K_{s+1}+X\right)\left(K_{t}+K_{t+1}\right)}{\left(K_{1}+\cdots+K_{t}\right)\left(K_{t+1}+\cdots+K_{s+1}\right)} & \\
& \left.\cdot f\left(t ;-K_{t+1}-\cdots-K_{s+1},-K_{t+1}-\cdots-K_{s+1}\right)\right|_{K_{t} \rightarrow K_{t}+\cdots+K_{s+1}} \\
& \quad-\left.\frac{\left(K_{s+1}+X\right)\left(K_{s}+K_{s+1}+Y\right)}{\left(K_{1}+\cdots+K_{s}\right) K_{s+1}} f\left(s ;-K_{s+1},-K_{s+1}\right)\right|_{K_{s} \rightarrow K_{s}+K_{s+1}} .
\end{aligned}
$$

Now we may use the induction hypothesis, so that we arrive at 


$$
\begin{gathered}
f(s+1 ; X, Y)=\left(K_{1}+\cdots+K_{s+1}+X\right) \\
-\sum_{t=1}^{s-1} \frac{\left(K_{t+1}+\cdots+K_{s+1}+X\right)\left(K_{t}+K_{t+1}\right)}{\left(K_{1}+\cdots+K_{t}\right)\left(K_{t+1}+\cdots+K_{s+1}\right)} \\
\cdot \frac{\left(K_{t+1}+\cdots+K_{s+1}\right)\left(K_{1}+\cdots+K_{t-1}+K_{t}\right)}{K_{2}+\cdots+K_{s+1}} \\
\quad-\frac{\left(K_{s+1}+X\right)\left(K_{s}+K_{s+1}+Y\right)}{\left(K_{1}+\cdots+K_{s}\right) K_{s+1}} \frac{K_{s+1}\left(K_{1}+\cdots+K_{s-1}+K_{s}\right)}{K_{2}+\cdots+K_{s+1}} \\
=\left(K_{1}+\cdots+K_{s+1}+X\right)-\sum_{t=1}^{s-1} \frac{\left(K_{t+1}+\cdots+K_{s+1}+X\right)\left(K_{t}+K_{t+1}\right)}{K_{2}+\cdots+K_{s+1}} \\
-\frac{\left(K_{s+1}+X\right)\left(K_{s}+K_{s+1}+Y\right)}{K_{2}+\cdots+K_{s+1}} .
\end{gathered}
$$

By a routine calculation this simplifies to

$$
f(s+1 ; X, Y)=-\frac{X\left(K_{1}+\cdots+K_{s}\right)+Y\left(K_{s+1}+X\right)}{K_{2}+\cdots+K_{s+1}} .
$$

This is exactly the right-hand side of (2.14) with $s$ replaced by $s+1$.

This completes the proof of the Theorem 2.1.

We finish the present section by making Theorem 2.1 explicit in the four lowestorder special cases. The following formulas are written in a way which makes the self-adjointness of the sums obvious.

Example 2.1. The definition $\mathcal{M}_{4}=P_{4}-P_{2}^{2}$ is equivalent to $P_{4}=\mathcal{M}_{4}+\mathcal{M}_{2}^{2}$.

Example 2.2. $P_{6}=\mathcal{M}_{6}+2\left(\mathcal{M}_{2} \mathcal{M}_{4}+\mathcal{M}_{4} \mathcal{M}_{2}\right)+\mathcal{M}_{2}^{3}$.

Example 2.3. $P_{8}$ can be written in the form of the sum

$$
\mathcal{M}_{8}+3\left(\mathcal{M}_{2} \mathcal{M}_{6}+\mathcal{M}_{6} \mathcal{M}_{2}\right)+9 \mathcal{M}_{4}^{2}+3\left(\mathcal{M}_{2}^{2} \mathcal{M}_{4}+\mathcal{M}_{4} \mathcal{M}_{2}^{2}\right)+4 \mathcal{M}_{2} \mathcal{M}_{4} \mathcal{M}_{2}+\mathcal{M}_{2}^{4}
$$

of 8 terms.

Example 2.4. $P_{10}$ can be written in the form of the sum

$$
\begin{aligned}
& \mathcal{M}_{10}+4\left(\mathcal{M}_{2} \mathcal{M}_{8}+\right.\left.\mathcal{M}_{8} \mathcal{M}_{2}\right)+24\left(\mathcal{M}_{4} \mathcal{M}_{6}+\mathcal{M}_{6} \mathcal{M}_{4}\right) \\
&+6\left(\mathcal{M}_{2}^{2} \mathcal{M}_{6}+\mathcal{M}_{6} \mathcal{M}_{2}^{2}\right)+24\left(\mathcal{M}_{2} \mathcal{M}_{4}^{2}+\mathcal{M}_{4}^{2} \mathcal{M}_{2}\right)+9 \mathcal{M}_{2} \mathcal{M}_{6} \mathcal{M}_{2}+16 \mathcal{M}_{4} \mathcal{M}_{2} \mathcal{M}_{4} \\
&+4\left(\mathcal{M}_{2}^{3} \mathcal{M}_{4}+\mathcal{M}_{4} \mathcal{M}_{2}^{3}\right)+6\left(\mathcal{M}_{2}^{2} \mathcal{M}_{4} \mathcal{M}_{2}+\mathcal{M}_{2} \mathcal{M}_{4} \mathcal{M}_{2}^{2}\right)+\mathcal{M}_{2}^{5}
\end{aligned}
$$

of 16 terms.

These formulas invert the definitions of the low-order special cases $\mathcal{M}_{2 N}(N \leq 5)$ displayed in Appendix 12.1. 


\section{Residue FAMilies}

In the present section, we recall from [J09a the notion of residue families

$$
D_{2 N}^{r e s}(g ; \lambda): C^{\infty}(M \times[0, \varepsilon)) \rightarrow C^{\infty}(M)
$$

(see also [BJ10] for an introduction) and describe their main properties. In particular, we describe their recursive structure which finds its natural expression in the form of two systems of factorization identities.

The definition of $D_{2 N}^{r e s}(g ; \lambda)$ involves two ingredients:

- the renormalized volume coefficients $v_{2}(g), \cdots, v_{2 N}(g)$ and

- the formal asymptotic expansions of eigenfunctions of the Laplacian of a Poincaré-Einstein metric $g_{+}$relative to $g$.

We first recall the notion of Poincaré-Einstein metrics in the sense of Fefferman and Graham [FG85], [FG07]. Let $M$ be a manifold of dimension $n$ with a given metric $g$. On the space $M \times(0, \varepsilon)$ we consider metrics of the form

$$
g_{+}=r^{-2}\left(d r^{2}+g_{r}\right)
$$

where $g_{r}$ is a one-parameter family of metrics on $M$ so that $g_{0}=g$. Moreover, we require that for odd $n$ the tensor

$$
\operatorname{Ric}\left(g_{+}\right)+n g_{+}
$$

vanishes to infinite order along $M$, and that for even $n \geq 4$

$$
\operatorname{Ric}\left(g_{+}\right)+n g_{+}=O\left(r^{n-2}\right)
$$

together with a vanishing trace condition for $\operatorname{Ric}\left(g_{+}\right)+n g_{+}$to the order $r^{n-2}$. If $g_{r}$ is required to be even (in $r$ ) and $n$ is odd, these conditions uniquely determine the family $g_{r}$ for a general metric $g$. Similarly, for even $n$, the conditions uniquely determine the coefficients $g_{(2)}, \ldots, g_{(n-2)}, \tilde{g}_{(n)}$ and the trace of $g_{(n)}$ in the even power series

$$
g_{r}=g+r^{2} g_{(2)}+\cdots+r^{n-2} g_{(n-2)}+r^{n}\left(g_{(n)}+\log r \tilde{g}_{(n)}\right)+\cdots .
$$

Moreover, we have $\operatorname{tr}_{g}\left(\tilde{g}_{(n)}\right)=0$. Since for even $n$ (and general metrics) the constructions in the present paper will only depend on the terms $g_{(2)}, \ldots, g_{(n-2)}$ and $\operatorname{tr}_{g}\left(g_{(n)}\right)$ (which are uniquely determined by $g$ ), it will be convenient to define $g_{r}$ in this case by the finite sum

$$
g+r^{2} g_{(2)}+\cdots+r^{n-2} g_{(n-2)}+r^{n} g_{(n)} .
$$

For a Poincaré-Einstein metric $g_{+}$of $g$, we consider the quotient

$$
v(r)=\frac{\operatorname{vol}\left(g_{r}\right)}{\operatorname{vol}(g)} \in C^{\infty}(M)
$$

of volume forms on $M$. Then

$$
\operatorname{dvol}\left(g_{+}\right)=r^{-n-1} v(r) \operatorname{dvol}(g) d r .
$$

In particular, for even $n$, we have the even expansion

$$
v(r)=1+r^{2} v_{2}+r^{4} v_{4}+\cdots+r^{n} v_{n}+\cdots .
$$


The coefficients $v_{2 j} \in C^{\infty}(M)$ in this expansion are called renormalized volume (or holographic) coefficients. The coefficients $v_{2 j}$ are intriguing scalar-valued Riemannian curvature invariants (see [G09, J09a and the references therein). We also define

$$
w(r)=\sqrt{v(r)}=1+r^{2} w_{2}+r^{4} w_{4}+\cdots+r^{n} w_{n}+\cdots .
$$

Now let $i: M \hookrightarrow M \times[0, \varepsilon)$ denote the embedding $i(m)=(m, 0)$. We consider formal solutions

$$
u(\cdot, r) \sim \sum_{j \geq 0} r^{\lambda+2 j} \mathcal{T}_{2 j}(g ; \lambda)(f)(\cdot), \mathcal{T}_{0}(f)=f \in C^{\infty}(M)
$$

of the eigen-equation

$$
-\Delta_{g_{+}} u=\lambda(n-\lambda) u, \lambda \in \mathbb{C} .
$$

The coefficients in the expansion (3.5) are given by rational families $\mathcal{T}_{2 j}(g ; \lambda)$ (in $\lambda$ ) of differential operators acting on the "boundary value" $f$ of $u$. For example, the first two families are

$$
\mathcal{T}_{2}(\lambda)=\frac{1}{2(n-2-2 \lambda)}(\Delta-\lambda \mathrm{J})
$$

and

$$
\begin{aligned}
\mathcal{T}_{4}(\lambda)=\frac{1}{8(n-2-2 \lambda)(n-4-2 \lambda)}[(\Delta-(\lambda+2) \mathrm{J})(\Delta-\lambda \mathrm{J}) \\
\left.\quad+\lambda(2 \lambda-n+2)|\mathrm{P}|^{2}+2(2 \lambda-n+2) \delta(\mathrm{P} d)+(2 \lambda-n+2)(d \mathrm{~J}, d)\right] .
\end{aligned}
$$

Now for general $g$, even $n$ and $2 N \leq n$, we define

$$
D_{2 N}^{r e s}(g ; \lambda)=2^{2 N} N !\left[\left(-\frac{n}{2}-\lambda+2 N-1\right) \cdots\left(-\frac{n}{2}-\lambda+N\right)\right] \delta_{2 N}(g ; \lambda+n-2 N)
$$

with

$$
\delta_{2 N}(g ; \lambda)=\sum_{j=0}^{N} \frac{1}{(2 N-2 j) !}\left[\mathcal{T}_{2 j}^{*}(g ; \lambda) v_{0}+\cdots+\mathcal{T}_{0}^{*}(g ; \lambda) v_{2 j}\right] i^{*}(\partial / \partial r)^{2 N-2 j}
$$

Here the holographic coefficients act as multiplication operators, and $\mathcal{T}_{2 j}^{*}(g ; \lambda)$ denotes the formal adjoint of the differential operator $\mathcal{T}_{2 j}(g ; \lambda)$ on $C^{\infty}(M)$ with respect to the metric $g$. Note that $D_{0}^{r e s}(g ; \lambda)=i^{*}$. The main role of the polynomial pre-factor in (3.6) is to remove the poles (see also (3.13)). Similarly, we define $D_{2 N}^{r e s}(g ; \lambda), N \geq 1$ for general $g$ and odd $n$. It is obvious that the definition of $D_{2 N}^{r e s}(\lambda)$ only requires to solve the eigen-equation approximately up to the order $r^{\lambda+2 N}$.

We also recall that, for a locally conformally flat metric $g$, the metric

$$
r^{-2}\left(d r^{2}+g-r^{2} \mathrm{P}+r^{4} / 4 \mathrm{P}^{2}\right)
$$

is a Poincaré-Einstein metric relative to $g$ (see Chapter 7 of [FG07] or Section 6.14 of [J09a]). In that case, residue families are well-defined for any $N \geq 1$.

The residue families $D_{2 N}^{r e s}(g ; \lambda)$ admit an interpretation as obstructions to the extension of eigenfunctions of $\Delta_{g_{+}}$through the boundary $r=0$. These obstructions 
appear as residues of associated meromorphic families of distributions. It is the latter relation which motivates the name. For later use, we need to recall the precise statement. Let $u \in C^{\infty}(X), X=(0, \varepsilon) \times M$ be an eigenfunction

$$
-\Delta_{g_{+}} u=\mu(n-\mu) u
$$

with boundary value $f \in C^{\infty}(M)$. Let $\varphi \in C_{0}^{\infty}(\bar{X})$ be a test function. We consider the integral

$$
\int_{X} r^{\lambda} u \varphi d v o l\left(r^{2} g_{+}\right)
$$

It is holomorphic for $\lambda$ with sufficiently large real part and admits a meromorphic continuation with a simple pole at $\lambda=-\mu-1-2 N$. Then

$$
\operatorname{Res}_{\lambda=-\mu-1-2 N}\left(\int_{X} r^{\lambda} u \varphi d v o l\left(r^{2} g_{+}\right)\right)=\int_{M} f \delta_{2 N}(\lambda+n-2 N)(\varphi) d v o l(g) .
$$

For full details we refer to [J09a] and [BJ10].

Now residue families have the following basic properties.

- $D_{2 N}^{r e s}(g ; \lambda)$ is a polynomial in $\lambda$ of degree $N$.

- $D_{2 N}^{r e s}(g ;-n / 2+N)=P_{2 N}(g) i^{*}$.

- $D_{2 N}^{r e s}(g ; \lambda)$ satisfies a certain conformal covariance law under $g \mapsto e^{2 \varphi} g$.

The second of these properties is contained in a system of $N$ factorization identities which are satisfied by the family $D_{2 N}^{r e s}(g ; \lambda)$. There are actually two such systems. The first system is given by the following result.

Theorem 3.1. Assume that $2 N \leq n$ for even $n$ and $N \geq 1$ for odd $n$. Then the factorization relations

$$
D_{2 N}^{r e s}\left(g ;-\frac{n}{2}+2 N-j\right)=P_{2 j}(g) \circ D_{2 N-2 j}^{r e s}\left(g ;-\frac{n}{2}+2 N-j\right)
$$

for $j=1, \ldots, N$ hold true for any metric $g$.

Next, we denote by

$$
\bar{P}_{2 N}(g)=P_{2 N}\left(d r^{2}+g_{r}\right)
$$

the GJMS-operator of order $2 N$ for the conformal compactification $\bar{g}=d r^{2}+g_{r}$ of $g_{+}$ on $X=M \times[0, \varepsilon)$. In these terms, the second system of factorization identities is given by the following result.

Theorem 3.2. Assume that $2 N \leq n$ for even $n$ and $N \geq 1$ for odd $n$. Then the factorization relations

$$
D_{2 N}^{r e s}\left(g ;-\frac{n+1}{2}+j\right)=D_{2 N-2 j}^{r e s}\left(g ;-\frac{n+1}{2}-j\right) \circ \bar{P}_{2 j}(g)
$$

for $j=1, \ldots, N$ hold true for any metric $g$.

We recall that, for general metrics on even-dimensional manifolds, the existence of associated Poincaré-Einstein metrics is obstructed. As a consequence, GJMSoperators for general metrics are only defined for orders which do not exceed the dimension of the underlying space. This is the reason for the condition $2 N \leq n$ in both theorems. However, in odd dimensions there are no obstructions. 
For odd $n$, the factorizations (3.11) involve the GJMS-operators of all orders $2 N \geq$ 2 on the space $X=M \times[0, \varepsilon)$ of even dimension $n+1$ for the metric $\bar{g}=d r^{2}+$ $g_{r}$. Nevertheless, these operators are well-defined. Indeed, Theorem 7.2 yields a formula for a Poincaré-Einstein metric for $\bar{g}$ to any order. Applying the construction of [GJMS92] defines the desired operators $\bar{P}_{2 N}$ for all $N \geq 1$. These are conjugate to the GJMS-operators for the Einstein metric $r^{-2} \bar{g}$.

Note also that for locally conformally flat metrics, Theorems $3.1-(3.2)$ extend to all $N \geq 1$.

The systems (3.9) and (3.11) contain the relations

$$
D_{2 N}^{r e s}\left(g,-\frac{n}{2}+N\right)=P_{2 N}(g) i^{*} \quad \text { and } \quad D_{2 N}^{r e s}\left(g ;-\frac{n+1}{2}+N\right)=i^{*} \bar{P}_{2 N}(g)
$$

as the respective special cases $j=N$.

In J09a, we established the factorizations in the system (3.9) for all metrics (if $2 N \leq n$ for even $n$ ) and the factorizations in the system (3.11) for locally conformally flat metrics. In J09a, we also confirmed the factorizations in the system (3.11) for $N=1,2$ and the factorization $j=1$ in the system (3.11) for $N=3$ by direct calculations. For locally conformally flat metrics, both systems of identities actually follow from their versions in the flat case by the conformal covariance of the families (see Theorem 6.6.3 in [J09a] and Theorem 1.5.3 in [BJ10]). Alternatively, the system (3.9) is a consequence of the identification of GJMS-operators in the asymptotic expansions of eigenfunctions of the Laplacian of Poincaré-Einstein metrics GZ03. The latter argument extends to general metrics. We continue with the description of the details of that proof.

Proof of Theorem 3.1. We use the fact that

$$
\mathcal{T}_{2 k}(\lambda)=\frac{1}{2^{2 k} k !\left(\frac{n}{2}-\lambda-1\right) \cdots\left(\frac{n}{2}-\lambda-k\right)} P_{2 k}(\lambda)
$$

with a holomorphic family $P_{2 k}(\lambda)$ of the form $\Delta^{k}+\cdots$ (see [GZ03], [J09a], [BJ10]). Hence we can write the family $D_{2 N}^{r e s}(\lambda)$ in the form

$$
\begin{aligned}
& \sum_{l=0}^{N-k} \sum_{k=0}^{N} \frac{2^{2 N} N !}{2^{2 k} k !} \frac{1}{(2 N-2 k-2 l) !} \\
& \times\left(-\frac{n}{2}-\lambda+2 N-k-1\right) \cdots\left(-\frac{n}{2}-\lambda+N\right) P_{2 k}^{*}(\lambda+n-2 N) v_{2 l} i^{*}\left(\frac{\partial^{2}}{\partial r^{2}}\right)^{N-k-l} .
\end{aligned}
$$

Since the families $P_{2 k}(\lambda)$ are polynomials of degree $k$ in $\lambda$ (which easily can be proved by induction), (3.13) shows that $D_{2 N}^{\text {res }}(\lambda)$ is a polynomial of degree $N$ in $\lambda$. Similarly, for the family $D_{2 N-2 j}^{r e s}(\lambda)$, we find the formula

$$
\begin{aligned}
& \sum_{l=0}^{N-j-k} \sum_{k=0}^{N-j} \frac{2^{2 N-2 j}(N-j) !}{2^{2 k} k !} \frac{1}{(2 N-2 j-2 k-2 l) !} \\
& \quad \times\left(-\frac{n}{2}-\lambda+2 N-2 j-k-1\right) \cdots\left(-\frac{n}{2}-\lambda+N-j\right)
\end{aligned}
$$




$$
\times P_{2 k}^{*}(\lambda+n-2(N-j)) v_{2 l} i^{*}\left(\frac{\partial^{2}}{\partial r^{2}}\right)^{N-j-k-l} .
$$

But for the value $\lambda=-\frac{n}{2}+2 N-j$, the non-trivial contributions in (3.13) satisfy $N \geq k \geq j$. Hence by an index shift the sum simplifies to

$$
\begin{aligned}
\sum_{l=0}^{N-k-j} \sum_{k=0}^{N-j} \frac{2^{2 N} N !}{2^{2 k+2 j}(k+j) !} \frac{1}{(2 N-2 k-2 j-2 l) !} \frac{(N-j) !}{k !}(-1)^{N-k-j} & \\
& \times P_{2 k+2 j}^{*}\left(\frac{n}{2}-j\right) v_{2 l} i^{*}\left(\frac{\partial^{2}}{\partial r^{2}}\right)^{N-k-j-l} .
\end{aligned}
$$

On the other hand, for $\lambda=-\frac{n}{2}+2 N-j$, the sum (3.14) reduces to

$$
\begin{aligned}
& \sum_{l=0}^{N-j-k} \sum_{k=0}^{N-j} \frac{2^{2 N-2 j}(N-j) !}{2^{2 k} k !} \frac{1}{(2 N-2 j-2 k-2 l) !} \frac{N !}{(k+j) !}(-1)^{N-k-j} \\
& \times P_{2 k}^{*}\left(\frac{n}{2}+j\right) v_{2 l} i^{*}\left(\frac{\partial^{2}}{\partial r^{2}}\right)^{N-j-k-l}
\end{aligned}
$$

Now we use the fact that the families $P_{2 N}(\lambda)$ obey the factorization relations

$$
P_{2 N}\left(\frac{n}{2}-k\right)=P_{2 N-2 k}\left(\frac{n}{2}+k\right) P_{2 k}
$$

for $N \geq k$ ([J09a], Theorem 6.11.18). For $k=N$, these relations state that

$$
P_{2 N}\left(\frac{n}{2}-N\right)=P_{2 N}
$$

Eq. (3.18) is the fundamental connection between GJMS-operators and formal asymptotic expansions of eigenfunctions of $\Delta_{g_{+}}$(see [GZ03]). In order to prove (3.17), we recall the structure of the asymptotic expansions of generalized eigenfunctions of $-\Delta_{g_{+}}$for generic eigenvalues. These have the form

$$
\sum_{j \geq 0} \mathcal{T}_{2 j}(n-\lambda)(f) r^{n-\lambda+2 j}+\sum_{j \geq 0} \mathcal{T}_{2 j}(\lambda) \mathcal{S}(\lambda)(f) r^{\lambda+2 j}
$$

where $\mathcal{S}(\lambda)$ is the scattering operator; here we use the conventions of [J09a]. Now the contributions

$$
\mathcal{T}_{2 N}(n-\lambda)(f) r^{n-\lambda+2 N} \quad \text { and } \quad \mathcal{T}_{2 N-2 k}(\lambda) \mathcal{S}(\lambda)(f) r^{\lambda+2 N-2 k}
$$

both have a simple pole at $\lambda=\frac{n}{2}+k$; note that $\mathcal{T}_{2 N-2 k}(\lambda)$ is regular at $\lambda=\frac{n}{2}+k$. The cancellation of poles in the sum implies the relation

$$
\operatorname{Res}_{\frac{n}{2}-k}\left(\mathcal{T}_{2 N}(\lambda)\right) r^{\frac{n}{2}+2 N-k}+\mathcal{T}_{2 N-2 k}\left(\frac{n}{2}+k\right) \operatorname{Res} \frac{n}{2}+k(\mathcal{S}(\lambda)) r^{\frac{n}{2}+2 N-k}=0 .
$$

But since $\operatorname{Res}_{\frac{n}{2}+k}(\mathcal{S}(\lambda))$ is proportional to $P_{2 k}$ ([GZ03]), we have proved that both sides of (3.17) are proportional. Since both sides are of the form $\Delta^{N}+\cdots$, this proves the equality. In particular, by taking adjoints, (3.17) shows that

$$
P_{2 k+2 j}^{*}\left(\frac{n}{2}-j\right)=P_{2 j}^{*} P_{2 k}^{*}\left(\frac{n}{2}+j\right)
$$


for $0 \leq k \leq N-j$. Therefore, the self-adjointness $P_{2 j}^{*}=P_{2 j}$ implies that the sum (3.15) coincides with the product of $P_{2 j}$ and (3.16). The proof is complete.

We continue with the

Proof of Theorem 3.2. The proof rests on the interpretation of residue families as residues as in (3.8). We set

$$
\mu=\frac{n-1}{2}+M-2 N \quad \text { with } \quad M=1, \ldots, N .
$$

We choose an arbitrary $f \in C^{\infty}(M)$. We consider formal approximate eigenfunctions of the Laplacian $-\Delta_{g_{+}}$for the eigenvalue $\mu(n-\mu)$ and with boundary value $f$. More precisely, for odd $n$ and $N \geq 1$ as well as for even $n$ and $N \leq \frac{n}{2}$, we let $u$ be the sum $\sum_{j=0}^{N} r^{\mu+2 j} a_{2 j}(\mu)$ with $a_{0}=f$ so that

$$
-\Delta_{g_{+}} u-\mu(n-\mu) u=O\left(r^{\mu+2 N+2}\right) \text {. }
$$

Then the coefficients $a_{2 j}(\mu)(j=1, \ldots, N)$ are uniquely determined and are given by the differential operators $\mathcal{T}_{2 j}(\mu)$ acting on $f$. Eq. (3.12) shows that the regularity of the families $\mathcal{T}_{2 j}(\lambda)$ at $\lambda=\mu$ is guaranteed by (3.21). In order to simplify the following arguments, we shall suppress the minor modifications caused by the fact that $u$ is only an approximate eigenfunction. Now

$$
P_{2 M}\left(g_{+}\right)(u)=\left(\prod_{j=0}^{2 M-1}(2 N-j)\right) u \stackrel{\text { def }}{=} \kappa u .
$$

In fact, since $g_{+}$is Einstein with scalar curvature $-n(n+1)$, the product formula (see [Go06] and [BJ10], Theorem 1.3.7)

$$
P_{2 M}\left(g_{+}\right)=\prod_{j=\frac{n+1}{2}}^{\frac{n+1}{2}+M-1}\left(\Delta_{g_{+}}+j(n-j)\right)
$$

implies that $P_{2 M}\left(g_{+}\right)$acts on $u$ by the scalar

$$
\begin{aligned}
& \prod_{j=\frac{n+1}{2}}^{\frac{n+1}{2}+M-1}(-\mu(n-\mu)+j(n-j)) \\
= & \prod_{k=0}^{M-1}\left(-\left(\frac{n-1}{2}+M-2 N\right)\left(\frac{n+1}{2}-M+2 N\right)+\left(\frac{n+1}{2}+k\right)\left(\frac{n-1}{2}-k\right)\right) \\
= & \prod_{k=0}^{M-1}(M-2 N+k)(M-2 N-1-k) \\
= & \prod_{j=0}^{2 M-1}(2 N-j) .
\end{aligned}
$$

This proves (3.22). Note that $\kappa \neq 0$. Next, the definition (3.6) gives 


$$
\begin{aligned}
D_{2 N}^{r e s}\left(g ;-\frac{n+1}{2}+M\right) & \\
& =2^{2 N} N !\left[\left(-\frac{1}{2}+2 N-M\right) \cdots\left(\frac{1}{2}+N-M\right)\right] \delta_{2 N}(g ; \mu)
\end{aligned}
$$

and

$$
\begin{aligned}
D_{2 N-2 M}^{r e s} & \left(g ;-\frac{n+1}{2}-M\right) \\
& =2^{2 N-2 M}(N-M) !\left[\left(-\frac{1}{2}+2 N-M\right) \cdots\left(\frac{1}{2}+N\right)\right] \delta_{2 N-2 M}(g ; \mu) .
\end{aligned}
$$

We observe that the quotient of the overall factors on the right-hand sides of (3.23) and (3.24) equals

$$
2^{2 M} \frac{N !}{(N-M) !}\left(-\frac{1}{2}+N\right) \cdots\left(\frac{1}{2}+N-M\right)=\kappa
$$

with $\kappa$ as defined in (3.22). Now (3.22) implies

$$
r^{\lambda} u=r^{\lambda+\frac{n+1}{2}+M}\left(r^{-\frac{n+1}{2}-M} u\right)=r^{\lambda+\frac{n+1}{2}+M} \kappa^{-1}\left(r^{-\frac{n+1}{2}-M} P_{2 M}\left(g_{+}\right)(u)\right) .
$$

Therefore, by the conformal covariance of $P_{2 M}$, we have

$$
r^{\lambda} u=r^{\lambda+\frac{n+1}{2}+M} \kappa^{-1} \bar{P}_{2 M}(g)\left(r^{-\frac{n+1}{2}+M} u\right) .
$$

Now let $\varphi \in C_{0}^{\infty}(X)$. We apply the identity (3.26) to rewrite the right-hand side of

$$
\int_{M} f \delta_{2 N}(g ; \mu)(\varphi) \operatorname{dvol}(g)=\operatorname{Res}_{\lambda=-\mu-1-2 N}\left(\int_{X} r^{\lambda} u \varphi d v o l(\bar{g})\right)
$$

(see Eq. (3.8)) in the form

$$
\kappa^{-1} \operatorname{Res}_{\lambda=-\mu-1-2 N}\left(\int_{X} \bar{P}_{2 M}(g)\left(r^{-\frac{n+1}{2}+M} u\right) r^{\lambda+\frac{n+1}{2}+M} \varphi d v o l(\bar{g})\right) .
$$

Now we use that $\bar{P}_{2 M}(g)=P_{2 M}(\bar{g})$ is self-adjoint with respect to the volume form of $\bar{g}$ and apply partial integration. Since boundary terms are holomorphic (in $\lambda$ ), they do not contribute to the residue. Hence (3.27) equals

$$
\kappa^{-1} \operatorname{Res}_{\lambda=-\mu-1-2 N}\left(\int_{X} r^{-\frac{n+1}{2}+M} u \bar{P}_{2 M}(g)\left(r^{\lambda+\frac{n+1}{2}+M} \varphi\right) d v o l(\bar{g})\right) .
$$

But the function

$$
\lambda \mapsto \bar{P}_{2 M}(g)\left(r^{\lambda+\frac{n+1}{2}+M} \varphi\right)-r^{\lambda+\frac{n+1}{2}+M} \bar{P}_{2 M}(g)(\varphi)
$$

vanishes at $\lambda=-\mu-1-2 N=-\frac{n+1}{2}-M$. It follows that the latter residue coincides with

$$
\kappa^{-1} \operatorname{Res}_{\lambda=-\mu-1-2 N}\left(\int_{X} r^{\lambda+2 M} u \bar{P}_{2 M}(g)(\varphi) \operatorname{dvol}(\bar{g})\right) .
$$

Thus, we conclude that

$$
\int_{M} f \delta_{2 N}(g ; \mu)(\varphi) d v o l(g)
$$


equals

$$
\kappa^{-1} \operatorname{Res}_{\lambda=-\mu-1-(2 N-2 M)}\left(\int_{X} r^{\lambda} u \bar{P}_{2 M}(g)(\varphi) \operatorname{dvol}(\bar{g})\right) .
$$

By (3.8), the latter expression can be written in the form

$$
\kappa^{-1} \int_{M} f \delta_{2 N-2 M}(g ; \mu)\left(\bar{P}_{2 M}(g)(\varphi)\right) \operatorname{dvol}(g) .
$$

But since $f \in C^{\infty}(M)$ is arbitrary, we have proved that

$$
\kappa \delta_{2 N}(g ; \mu)=\delta_{2 N-2 M}(g ; \mu) \bar{P}_{2 M}(g) .
$$

By (3.25), this identity is equivalent to the assertion.

We finish this section with two remarks. The proofs of the factorization identities utilize the condition that $g_{+}$is Einstein in two ways. In fact, the Einstein condition enters into the proof of Theorem 3.1 through the connection between asymptotic expansions of eigenfunctions of the Laplacian on $X=M \times(0, \varepsilon)$ and GJMS-operators on $M$. On the other hand, the proof of Theorem 3.2 rests on the fact that all GJMSoperators for Einstein metrics on $X$ act by scalars on eigenfunctions of the Laplacian (which is a consequence of the product formula for these operators). Finally, the residue families $D_{2 N}^{r e s}(\lambda)$ should be regarded as curved analogs of (differential) intertwining operators. From that perspective, the factorization identities appear as curved analogs of the fact that, in multiplicity-free decompositions, spaces of intertwining operators are one-dimensional.

\section{Residue families in terms of GJMS-OPERATORS}

In the present section, we establish a fundamental representation formula for residue families in terms of GJMS-operators. Let

$$
\pi_{2 N}(x) \stackrel{\text { def }}{=} x(x-1) \cdots(x-N+1)\left(x-\frac{1}{2}+N\right) \cdots\left(x+\frac{1}{2}\right) .
$$

Then

$$
\pi_{2 N}^{-1}(0)=\{0,1, \ldots, N-1\} \cup\left\{-\frac{1}{2}, \ldots,-N+\frac{1}{2}\right\} .
$$

We also set $m_{\bar{I}}=-m_{I}$. In these terms, the main result can be stated as follows.

Theorem 4.1. The residue family $D_{2 N}^{r e s}(\lambda)$ coincides with the sum of

$$
\begin{aligned}
& -(-1)^{N} \frac{2^{2 N-1}}{(2 N-1) !} \sum_{|I|=N} \frac{\pi_{2 N}\left(\lambda+\frac{n}{2}-N\right)}{\left(\lambda+\frac{n}{2}-2 N+I_{l}\right)} m_{I} P_{2 I}, \\
& -(-1)^{N} \frac{2^{2 N-1}}{(2 N-1) !} \sum_{|J|=N} \frac{\pi_{2 N}\left(\lambda+\frac{n}{2}-N\right)}{\left(\lambda+\frac{n+1}{2}-J_{r}\right)} m_{\bar{J}} \bar{P}_{2 J}
\end{aligned}
$$

and

$$
(-1)^{N} \frac{2^{2 N-1}}{(2 N-1) !}
$$




$$
\times \sum_{|I|+|J|=N} \frac{\pi_{2 N}\left(\lambda+\frac{n}{2}-N\right)}{\left(\lambda+\frac{n}{2}-2 N+I_{l}\right)\left(\lambda+\frac{n+1}{2}-J_{r}\right)} \frac{N !(N-1) !}{|I| !(|I|-1) !|J| !(|J|-1) !} m_{I} m_{\bar{J}} P_{2 I} \bar{P}_{2 J} .
$$

Some comments are in order.

We have slightly simplified the formulation of the representation formula by omitting all compositions with the pull-back $i^{*}$. In particular, $P_{2 I} \bar{P}_{2 J}$ means $P_{2 I} i^{*} \bar{P}_{2 J}$. We shall use this convention throughout from now on.

$I_{l}$ and $J_{r}$ denote the respective most left and most right entries of the compositions $I$ and $J$. Note also that the formulas are valid for any $\lambda \in \mathbb{C}$ since all fractions actually are polynomials in $\lambda$. In fact,

$$
\lambda+\frac{n}{2}-2 N+I_{l}=\left(\lambda+\frac{n}{2}-N\right)-\left(N-I_{l}\right)
$$

with $\left(N-I_{l}\right) \in\{0,1, \ldots, N-1\} \subset \pi_{2 N}^{-1}(0)$ and

$$
\lambda+\frac{n+1}{2}-J_{r}=\left(\lambda+\frac{n}{2}-N\right)+\left(N+\frac{1}{2}-J_{r}\right)
$$

with $-\left(N+\frac{1}{2}-J_{r}\right) \in\left\{-\frac{1}{2}, \ldots,-N+\frac{1}{2}\right\} \subset \pi_{2 N}^{-1}(0)$. The sums (4.2) and (4.3) can be viewed as degenerate special cases of (4.4). The formula in Theorem 4.1 also reflects a certain symmetry of the families $D_{2 N}^{r e s}(\lambda)$. In fact, the polynomials $\pi_{2 N}$ satisfy the symmetry relations

$$
\pi_{2 N}(\lambda)=\pi_{2 N}\left(-\lambda-\frac{1}{2}\right)
$$

Now using Theorem 4.1 these symmetry relations imply that

$$
\sigma D_{2 N}^{r e s}\left(\lambda-\frac{n}{2}+N\right)=D_{2 N}^{r e s}\left(-\lambda-\frac{n+1}{2}+N\right)
$$

where $\sigma$ maps $P_{2 a} \bar{P}_{2 b}$ into $P_{2 b} \bar{P}_{2 a}$. But the relation (4.5) directly follows from the factorization relations for residue families using an induction on $N$.

The proof of Theorem 4.1 will be given in Section 5. The idea of the proof is the following. For any $N \geq 1$, we define a family $D_{2 N}(\lambda)$ by their Taylor expansions in terms of GJMS-operators. We prove that these families coincide with the sum of (4.2), (4.3) and (4.4), and satisfy the same systems of factorization identities as the residue families $D_{2 N}^{r e s}(\lambda)$ (see Section 3 ).

We continue with the definition of the families

$$
D_{2 N}(\lambda) \stackrel{\text { def }}{=} \sum_{k=1}^{2 N} d_{2 N}^{(k)}\left(\lambda+\frac{n}{2}-N\right)^{2 N-k} .
$$

The coefficients $d_{2 N}^{(k)}$ are certain linear combinations of compositions of GJMS-operators $P_{2 M}$ and $\bar{P}_{2 M}$ for $1 \leq M \leq N$. In order to define these coefficients, we distinguish between

- compositions of GJMS-operators for $g$,

- compositions of GJMS-operators for $\bar{g}$ and

- mixed compositions of GJMS-operators for $g$ and $\bar{g}$.

The respective multiplicities of these compositions will be defined and studied in the following three subsections. 
4.1. The pure $P$-terms. Let

$$
m_{(a, I)}^{(k)}, a+|I|=N, a \geq 1
$$

be the coefficient of $P_{2 a} P_{2 I}$ in the coefficient $d_{2 N}^{(k)}$. Here $I$ can be trivial, i.e., $a=N$. Then

$$
m_{(a, I)}^{(k)} \stackrel{\text { def }}{=}\left(\sum_{j=0}^{k-1} \eta_{2 N}^{(2 N-j-1)}|I|^{k-1-j}\right) m_{(a, I)}^{(1)}
$$

where

$$
\eta_{2 N}(x) \stackrel{\text { def }}{=} \frac{\pi_{2 N}(x)}{x}=\sum_{k=0}^{2 N-1} \eta_{2 N}^{(k)} x^{k}
$$

In particular, we find

$$
m_{(a, I)}^{(2 N)}=\left(\sum_{j=0}^{2 N-1} \eta_{2 N}^{(2 N-j-1)}|I|^{2 N-1-j}\right) m_{(a, I)}^{(1)}=\eta_{2 N}(|I|) m_{(a, I)}^{(1)} .
$$

Hence

$$
m_{(a, I)}^{(2 N)}= \begin{cases}0 & \text { for } 1 \leq|I| \leq N-1 \\ \eta_{2 N}(0) m_{(N)}^{(1)} & \text { for }|I|=0\end{cases}
$$

A calculation shows that

$$
\eta_{2 N}(0)=-(-1)^{N} 2^{-(2 N-1)}(2 N-1) !=\pi_{2 N}^{\prime}(0) .
$$

Finally, we have

$$
m_{I}^{(1)} \stackrel{\text { def }}{=}-(-1)^{N} \frac{2^{2 N-1}}{(2 N-1) !} m_{I}
$$

for all compositions $I$ of size $N$.

The relations (4.8), (4.9) and (4.10) show that the constant term of $D_{2 N}(\lambda)$ equals $P_{2 N}$. Hence the multiplicity $\left[P_{2 N}: D_{2 N}(\lambda)\right]$ of the total contribution of $P_{2 N}$ to $D_{2 N}(\lambda)$ is given by

$$
\left(\sum_{k=1}^{2 N}\left(\lambda+\frac{n}{2}-N\right)^{2 N-k} \eta_{2 N}^{(2 N-k)}\right) m_{(N)}^{(1)}=-(-1)^{N} \frac{2^{2 N-1}}{(2 N-1) !} \eta_{2 N}\left(\lambda+\frac{n}{2}-N\right) .
$$

More generally, for non-trivial $I$, the multiplicity $\left[P_{2 a} P_{2 I}: D_{2 N}(\lambda)\right]$ of the total contribution of $P_{2 a} P_{2 I}$ to $D_{2 N}(\lambda)$ is given by

$$
\left(\sum_{k=1}^{2 N-1}\left(\lambda+\frac{n}{2}-N\right)^{2 N-k}\left(\sum_{i=0}^{k-1} \eta_{2 N}^{(2 N-i-1)}|I|^{k-1-i}\right)\right) m_{(a, I)}^{(1)} .
$$

The latter double sum can be written in the form

$$
\left(\sum_{\substack{1 \leq i+k \leq 2 N-1 \\ 0 \leq i \leq 2 N-2,1 \leq k \leq 2 N-1}} \eta_{2 N}^{(i+k)}|I|^{i}\left(\lambda+\frac{n}{2}-N\right)^{k}\right) m_{(a, I)}^{(1)} .
$$

In order to determine that sum, we apply the following result. 
Lemma 4.1. For $x \neq y$,

$$
\sum_{\substack{1 \leq a+b \leq 2 N-1 \\ 1 \leq a \leq 2 N-1,0 \leq b \leq 2 N-2}} \eta_{2 N}^{(a+b)} x^{a} y^{b}=x\left(\frac{\eta_{2 N}(x)-\eta_{2 N}(y)}{x-y}\right) .
$$

Moreover,

$$
\sum_{\substack{1 \leq a+b \leq 2 N-1 \\ 1 \leq a \leq 2 N-1,0 \leq b \leq 2 N-2}} \eta_{2 N}^{(a+b)} M^{a+b-1}=\eta_{2 N}^{\prime}(M)
$$

for $M=1, \ldots, N-1$.

Proof. Let $x \neq y$. The left-hand side of (4.12) equals

$$
\begin{aligned}
\sum_{k=1}^{2 N-1} \eta_{2 N}^{(k)}\left(\sum_{a=1}^{k} x^{a} y^{k-a}\right) & =\sum_{k=1}^{2 N-1} \eta_{2 N}^{(k)}\left(\sum_{a=0}^{k} x^{a} y^{k-a}-y^{k}\right) \\
& =\sum_{k=1}^{2 N-1} \eta_{2 N}^{(k)}\left(\frac{x^{k+1}-y^{k+1}}{x-y}-y^{k}\right) .
\end{aligned}
$$

Using the definition of $\eta_{2 N}$, the latter sum simplifies to

$$
\frac{1}{x-y}\left(x\left(\eta_{2 N}(x)-\eta_{2 N}^{(0)}\right)-y\left(\eta_{2 N}(y)-\eta_{2 N}^{(0)}\right)\right)-\left(\eta_{2 N}(y)-\eta_{2 N}^{(0)}\right) .
$$

Now the first assertion follows by simplification. For $y=1, \ldots, N-1$, the second assertion follows by taking the limit $x \rightarrow y$.

Lemma 4.1 and $\eta_{2 N}(|I|)=0$ for $1 \leq|I| \leq N-1$ imply that the multiplicity $\left[P_{2 a} P_{2 I}: D_{2 N}(\lambda)\right]$ of the total contribution of $P_{2 a} P_{2 I}$ to $D_{2 N}(\lambda)$ is given by

$$
-(-1)^{N} \frac{2^{2 N-1}}{(2 N-1) !} \frac{\pi_{2 N}\left(\lambda+\frac{n}{2}-N\right)}{\left(\lambda+\frac{n}{2}-N-|I|\right)} m_{(a, I)},
$$

if $\lambda+\frac{n}{2}-N \neq|I|$, and

$$
-(-1)^{N} \frac{2^{2 N-1}}{(2 N-1) !} \pi_{2 N}^{\prime}(|I|) m_{(a, I)}
$$

if $\lambda+\frac{n}{2}-N=|I|$. Note that for trivial $I$ (4.14) specializes to (4.11). These results yield the sum in (4.2).

\subsection{The pure $\bar{P}$-terms. Let}

$$
m_{(\bar{I}, \bar{b})}^{(k)},|I|+b=N
$$

be the coefficient of $\bar{P}_{2 I} \bar{P}_{2 b}$ in the coefficient $d_{2 N}^{(k)}$. Here $I$ can be trivial, i.e., $b=N$. Then

$$
\begin{aligned}
& m_{(\bar{I}, \bar{b})}^{(k)} \\
& \quad \stackrel{\text { def }}{=}\left(\sum_{j=0}^{k-1} \tau_{2 N}^{(2 N-j-1)}\left(-|I|-\frac{1}{2}\right)^{k-1-j}+\frac{1}{2} \sum_{j=0}^{k-2} \tau_{2 N}^{(2 N-j-1)}\left(-|I|-\frac{1}{2}\right)^{k-2-j}\right) m_{(\bar{I}, \bar{b})}^{(1)},
\end{aligned}
$$


where

$$
\tau_{2 N}(x) \stackrel{\text { def }}{=} \frac{\pi_{2 N}(x)}{x+\frac{1}{2}}=\sum_{k=0}^{2 N-1} \tau_{2 N}^{(k)} x^{k} .
$$

Finally, we set $m_{\bar{I}}^{(1)}=-m_{I}^{(1)}$. In particular, we find

$$
\begin{aligned}
& m_{(\bar{I}, \bar{b})}^{(2 N)} / m_{(\bar{I}, \bar{b})}^{(1)} \\
& \quad=\left(\sum_{j=0}^{2 N-1} \tau_{2 N}^{2 N-j-1}\left(-|I|-\frac{1}{2}\right)^{2 N-1-j}+\frac{1}{2}\left(\sum_{j=0}^{2 N-2} \tau_{2 N}^{2 N-j-1}\left(-|I|-\frac{1}{2}\right)^{2 N-2-j}\right)\right) .
\end{aligned}
$$

Now the latter sum simplifies to

$$
-\frac{\pi_{2 N}\left(-|I|-\frac{1}{2}\right)}{|I|+\frac{1}{2}}
$$

It follows that the contribution of $\bar{P}_{2 N}$ to the constant term of $D_{2 N}(\lambda)$ vanishes. Hence for all $I$ (including trivial $I$ ), the multiplicity $\left[\bar{P}_{2 I} \bar{P}_{2 b}: D_{2 N}(\lambda)\right]$ of the total contribution of $\bar{P}_{2 I} \bar{P}_{2 b}$ to $D_{2 N}(\lambda)$ is given by the product of

$$
\begin{aligned}
& \sum_{k=1}^{2 N-1}\left(\lambda+\frac{n}{2}-N\right)^{2 N-k} \\
& \quad \times\left(\sum_{j=0}^{k-1} \tau_{2 N}^{(2 N-j-1)}\left(-|I|-\frac{1}{2}\right)^{k-1-j}+\frac{1}{2} \sum_{j=0}^{k-2} \tau_{2 N}^{(2 N-j-1)}\left(-|I|-\frac{1}{2}\right)^{k-2-j}\right)
\end{aligned}
$$

and

$$
m_{(\bar{I}, \bar{b})}^{(1)}=(-1)^{N} \frac{2^{2 N-1}}{(2 N-1) !} m_{(I, b)} .
$$

Now the sum (4.18) can be written in the form

$$
\begin{aligned}
\sum_{\substack{1 \leq a+b \leq 2 N-1 \\
1 \leq a \leq 2 N-1,0 \leq b \leq 2 N-2}} \tau_{2 N}^{(a+b)}\left(\lambda+\frac{n}{2}-N\right)^{a}\left(-|I|-\frac{1}{2}\right)^{b} \\
+\frac{1}{2} \sum_{\substack{2 \leq a+b \leq 2 N-1 \\
1 \leq a \leq 2 N-1,1 \leq b \leq 2 N-2}} \tau_{2 N}^{(a+b)}\left(\lambda+\frac{n}{2}-N\right)^{a}\left(-|I|-\frac{1}{2}\right)^{b-1} .
\end{aligned}
$$

In order to determine that sum, we apply the following result.

Lemma 4.2. For $x \neq y$,

$$
\sum_{\substack{1 \leq a+b \leq 2 N-1 \\ 1 \leq a \leq 2 N-1,0 \leq b \leq 2 N-2}} \tau_{2 N}^{(a+b)} x^{a} y^{b}+\frac{1}{2} \sum_{\substack{2 \leq a+b \leq 2 N-1 \\ 1 \leq a \leq 2 N-1,1 \leq b \leq 2 N-2}} \tau_{2 N}^{(a+b)} x^{a} y^{b-1}=\frac{y \pi_{2 N}(x)-x \pi_{2 N}(y)}{(x-y) y} .
$$

Proof. By an analog of Lemma 4.1, the first sum equals

$$
x\left(\frac{\tau_{2 N}(x)-\tau_{2 N}(y)}{x-y}\right) .
$$


For the second sum, we find

$$
\begin{aligned}
\sum_{k=2}^{2 N-1} \tau_{2 N}^{(k)}\left(\sum_{a=1}^{k-1} x^{a} y^{k-a-1}\right) & =\sum_{k=2}^{2 N-1}\left(\frac{x^{k}-y^{k}}{x-y}-y^{k-1}\right) \\
& =\frac{1}{x-y}\left(\sum_{k=2}^{2 N-1} \tau_{2 N}^{(k)} x^{k}-\tau_{2 N}^{(k)} y^{k}\right)-\sum_{k=2}^{2 N-1} \tau_{2 N}^{(k)} y^{k-1} \\
& =\frac{\tau_{2 N}(x)-\tau_{2 N}(y)}{x-y}-\frac{\tau_{2 N}(y)}{y}
\end{aligned}
$$

Hence the total sum equals

$$
\begin{aligned}
& x \frac{\tau_{2 N}(x)-\tau_{2 N}(y)}{x-y}+\frac{1}{2} \frac{\tau_{2 N}(x)-\tau_{2 N}(y)}{x-y}-\frac{1}{2} \frac{\tau_{2 N}(y)}{y} \\
& =\frac{y\left(x+\frac{1}{2}\right) \tau_{2 N}(x)-x\left(y+\frac{1}{2}\right) \tau_{2 N}(y)}{(x-y) y} \\
& =\frac{y \pi_{2 N}(x)-x \pi_{2 N}(y)}{(x-y) y} .
\end{aligned}
$$

The proof is complete.

Lemma 4.2 and $\pi_{2 N}\left(-|I|-\frac{1}{2}\right)=0$ for $0 \leq|I| \leq N-1$ imply that the multiplicity $\left[\bar{P}_{2 I} \bar{P}_{2 j}: D_{2 N}(\lambda)\right]$ of the total contribution of $\bar{P}_{2 I} \bar{P}_{2 j}$ to $D_{2 N}(\lambda)$ equals

$$
-(-1)^{N} \frac{2^{2 N-1}}{(2 N-1) !} \frac{\pi_{2 N}\left(\lambda+\frac{n}{2}-N\right)}{\lambda+\frac{n+1}{2}-N+|I|} m_{(\bar{I}, \bar{b})}
$$

if $\lambda+\frac{n+1}{2}-N+|I| \neq 0$, and

$$
-(-1)^{N} \frac{2^{2 N-1}}{(2 N-1) !} \pi_{2 N}^{\prime}\left(-|I|-\frac{1}{2}\right) m_{(\bar{I}, \bar{b})}
$$

if $\lambda+\frac{n+1}{2}-N+|I|=0$. In particular, $\bar{P}_{2 N}$ contributes to $D_{2 N}(\lambda)$ by

$$
-(-1)^{N} \frac{2^{2 N-1}}{(2 N-1) !} \frac{\pi_{2 N}\left(\lambda+\frac{n}{2}-N\right)}{\lambda+\frac{n+1}{2}-N} .
$$

These results yield the sum in (4.3).

\subsection{The mixed terms. Let}

$$
m_{(I, \bar{J})}^{(k)},|I|+|J|=N
$$

be the coefficient of $P_{2 I} \bar{P}_{2 J}$ in the coefficient $d_{2 N}^{(k)}$. Here both $I$ and $J$ are non-trivial. Then this coefficient is given by

$$
m_{(I, \bar{J})}^{(k)} \stackrel{\text { def }}{=} \mu_{\left(I_{l}, J_{r}\right)}^{(2 N-k)}(N) m_{(I, \bar{J})}^{(2)}
$$

where

$$
\frac{\pi_{2 N}(x)}{(x-N+a)\left(x+N-b+\frac{1}{2}\right)}=\sum_{k=0}^{2 N-2} \mu_{(a, b)}^{(k)}(N) x^{k} .
$$


Finally, we have

$$
m_{(I, \bar{J})}^{(2)} \stackrel{\text { def }}{=}-(-1)^{N} 2^{2 N-1} \frac{N !(N-1) !}{(2 N-1) !} \frac{m_{I} m_{J}}{|I| !(|I|-1) !|J| !(|J|-1) !} .
$$

Hence the mixed contributions to $D_{2 N}(\lambda)$ are given by the sum

$$
\sum_{|I|+|J|=N} \frac{\pi_{2 N}\left(\lambda+\frac{n}{2}-N\right)}{\left(\lambda+\frac{n}{2}-2 N+I_{l}\right)\left(\lambda+\frac{n+1}{2}-J_{r}\right)} m_{(I, \bar{J})}^{(2)} P_{2 I} \bar{P}_{2 J}
$$

or, equivalently,

$$
\begin{aligned}
& -(-1)^{N} 2^{2 N-1} \frac{N !(N-1) !}{(2 N-1) !} \\
& \quad \times \sum_{|I|+|J|=N} \frac{\pi_{2 N}\left(\lambda+\frac{n}{2}-N\right)}{\left(\lambda+\frac{n}{2}-2 N+I_{l}\right)\left(\lambda+\frac{n+1}{2}-J_{r}\right)} \frac{m_{I} m_{J}}{|I| !(|I|-1) !|J| !(|J|-1) !} P_{2 I} \bar{P}_{2 J} .
\end{aligned}
$$

Here $I_{l}$ and $J_{r}$ denote the most left and most right entries of the compositions $I$ and $J$, respectively. These formulas are valid for all $\lambda$ by reducing the fractions if necessary. These results yield the sum in (4.4).

\section{FACTORIZATION RELATiOns FOR $D_{2 N}(\lambda)$}

For the proof of Theorem 4.1, it suffices to prove that the families $D_{2 N}(\lambda)$ satisfy the factorization relations

and

$$
D_{2 N}\left(-\frac{n}{2}+2 N-j\right)=P_{2 j} D_{2 N-2 j}\left(-\frac{n}{2}+2 N-j\right), j=1, \ldots, N
$$

$$
D_{2 N}\left(-\frac{n+1}{2}+j\right)=D_{2 N-2 j}\left(-\frac{n+1}{2}-j\right) \bar{P}_{2 j}, j=1, \ldots, N .
$$

In fact, the relations in the systems (5.1) and (5.2) are analogs of the relations (3.9) and (3.11). But since both families $D_{2 N}(\lambda)$ and $D_{2 N}^{r e s}(\lambda)$ are polynomials of degree $\leq 2 N-1$ in $\lambda$, they are characterized by the respective systems of factorization identities.

A central role in the following arguments will be played by the identity

$$
\begin{aligned}
\left(2 N-j-\frac{1}{2}\right) \cdots\left(N-j+\frac{1}{2}\right) & \frac{(N-1) !}{(2 N-1) !} \\
= & \left(2 N-j-\frac{1}{2}\right) \cdots\left(N+\frac{1}{2}\right) 2^{-2 j} \frac{(N-j-1) !}{(2 N-2 j-1) !} .
\end{aligned}
$$

It is straightforward to verify this relation.

Now, in order to prove the factorizations (5.1) and (5.2), we compare the contributions of all possible types of products of GJMS-operators on both sides.

We start by proving that the contributions of the compositions

$$
P_{2 j} P_{2 I} \bar{P}_{2 J}, \quad j+|I|+|J|=N
$$

with non-trivial $I$ and $J$ on both sides of (5.1) coincide. By (4.24), the assertion is equivalent to the identity 


$$
\begin{aligned}
& \frac{\pi_{2 N}^{\prime}(N-j)}{\left(2 N+\frac{1}{2}-j-J_{r}\right)} \frac{2^{2 N-1}}{(2 N-1) !} \frac{N !(N-1) !}{(|I|+j) !(|I|+j-1) !|J| !(|J|-1) !} m_{(j, I)} m_{J} \\
& \quad=(-1)^{j} \frac{\pi_{2 N-2 j}(N)}{\left(j+I_{l}\right)\left(2 N+\frac{1}{2}-j-J_{r}\right)} \frac{2^{2 N-2 j-1}}{(2 N-2 j-1) !} \frac{(N-j) !(N-j-1) !}{|I|-1) !|J| !(|J|-1) !} m_{I} m_{J} .
\end{aligned}
$$

Now the identities

$$
\pi_{2 N}^{\prime}(N-j)=-(-1)^{j}(N-j) !(j-1) !\left(2 N-j-\frac{1}{2}\right) \cdots\left(N-j+\frac{1}{2}\right)
$$

and

$$
\pi_{2 N-2 j}(N)=\frac{N !}{j !}\left(2 N-j-\frac{1}{2}\right) \cdots\left(N+\frac{1}{2}\right)
$$

simplify the assertion to

$$
\begin{aligned}
(j-1) ! & \left(2 N-j-\frac{1}{2}\right) \cdots\left(N-j+\frac{1}{2}\right) \frac{1}{(2 N-1) !} \frac{N !(N-1) !}{(|I|+j) !(|I|+j-1) !} m_{(j, I)} \\
& =-\frac{N !}{j !}\left(2 N-j-\frac{1}{2}\right) \cdots\left(N+\frac{1}{2}\right) \frac{1}{j+I_{l}} 2^{-2 j} \frac{(N-j-1) !}{(2 N-2 j-1) !|I| !(|I|-1) !} m_{I} .
\end{aligned}
$$

But the definition of the multiplicities $m_{I}$ implies the relation

$$
m_{(j, I)}=-\frac{1}{j+I_{l}}\left(\begin{array}{c}
j+|I| \\
j
\end{array}\right)^{2} \frac{j|I|}{j+|I|} m_{I}=-\frac{1}{j+I_{l}} \frac{(j+|I|) !(j+|I|-1) !}{j !(j-1) !|I| !(|I|-1) !} m_{I} .
$$

It further simplifies the assertion to (5.3).

Next, we prove that the contributions of the compositions

$$
P_{2 I} \bar{P}_{2 J} \bar{P}_{2 j}, \quad|I|+|J|+j=N
$$

with non-trivial $I$ and $J$ on both sides of (5.2) coincide. By (4.24), the assertion is equivalent to

$$
\begin{aligned}
& \frac{\pi_{2 N}^{\prime}\left(-\frac{1}{2}+j-N\right)}{\left(-\frac{1}{2}-2 N+j+I_{l}\right)} \frac{2^{2 N-1}}{(2 N-1) !} \frac{N !(|I|) !(|I|-1) !(|J|+j) !(|J|+j-1) !}{(2 N) !} m_{I} m_{(J, j)} \\
& \quad=(-1)^{j} \frac{\pi_{2 N-2 j}\left(-\frac{1}{2}-N\right)}{\left(-J_{r}-j\right)\left(-\frac{1}{2}+j-2 N+I_{l}\right)} \frac{2^{2 N-2 j-1}}{(2 N-2 j-1) !} \frac{(N-j) !(N-j-(|I|-1) !|J| !(|J|-1) !}{\mid I I} m_{I} m_{J} .
\end{aligned}
$$

Now the identities

$$
\pi_{2 N}^{\prime}\left(-\frac{1}{2}+j-N\right)=(-1)^{j}(N-j) !(j-1) !\left(2 N-j-\frac{1}{2}\right) \cdots\left(N-j+\frac{1}{2}\right)
$$

and

$$
\pi_{2 N-2 j}\left(-\frac{1}{2}-N\right)=\frac{N !}{j !}\left(2 N-j-\frac{1}{2}\right) \cdots\left(N+\frac{1}{2}\right)
$$

simplify the assertion to

$$
\begin{aligned}
-(j-1) ! & \left(2 N-j-\frac{1}{2}\right) \cdots\left(N-j+\frac{1}{2}\right) \frac{1}{(2 N-1) !} \frac{N !(N-1) !}{(|J|+j) !(|J|+j-1) !} m_{(J, j)} \\
= & \frac{N !}{j !}\left(2 N-j-\frac{1}{2}\right) \cdots\left(N+\frac{1}{2}\right) \frac{1}{J_{r}+j} 2^{-2 j} \frac{(N-j-1) !}{(2 N-2 j-1) !|J| !(|J|-1) !} m_{J} .
\end{aligned}
$$


But the definition of the multiplicities $m_{I}$ implies the relation

$$
m_{(J, j)}=-\frac{1}{J_{r}+j}\left(\begin{array}{c}
j+|J| \\
j
\end{array}\right)^{2} \frac{j|J|}{j+|J|} m_{J}=-\frac{1}{J_{r}+j} \frac{(j+|J|) !(j+|J|-1) !}{j !(j-1) !|J| !(|J|-1) !} m_{J}
$$

which simplifies the assertion to (5.3).

Next, we prove that the contributions of

$$
P_{2 j} P_{2 I}, \quad j+|I|=N
$$

on both sides of (5.1) coincide. On the one hand, (4.15) shows that $P_{2 j} P_{2 I}$ contributes to

with the coefficient

$$
D_{2 N}\left(-\frac{n}{2}+2 N-j\right)
$$

$$
-(-1)^{N} \frac{2^{2 N-1}}{(2 N-1) !} \pi_{2 N}^{\prime}(N-j) m_{(j, I)} .
$$

By (5.4), the latter term equals

$$
(-1)^{N-j} 2^{2 N-1} \frac{(N-j) !(j-1) !}{(2 N-1) !}\left(2 N-j-\frac{1}{2}\right) \cdots\left(N-j+\frac{1}{2}\right) m_{(j, I)} .
$$

We compare this coefficient with the coefficient of the contribution of $P_{2 I}$ to

$$
D_{2 N-2 j}\left(-\frac{n}{2}+2 N-j\right) \text {. }
$$

For this purpose, we write $I$ in the form $\left(I_{l}, K\right)$ with a possibly trivial $K$. Let $K$ be non-trivial. Then $|K|=N-I_{l}-j$. Now by (4.14), the composition $P_{2 I_{l}} P_{2 K}$ contributes with the coefficient

$$
\frac{\pi_{2 N-2 j}\left(\lambda+\frac{n}{2}-(N-j)\right)}{\lambda+\frac{n}{2}-(N-j)-|K|} m_{\left(I_{l}, K\right)}^{(1)}=\frac{\pi_{2 N-2 j}(N)}{I_{l}+j} m_{\left(I_{l}, K\right)}^{(1)} .
$$

By (5.5), it equals

$$
-(-1)^{N-j} 2^{2(N-j)-1} \frac{N !}{(2 N-2 j-1) ! j !} \frac{1}{I_{l}+j}\left(2 N-j-\frac{1}{2}\right) \cdots\left(N+\frac{1}{2}\right) m_{\left(I_{l}, K\right)} .
$$

We claim that the latter coefficient coincides with

$$
(-1)^{N-j} 2^{2 N-1} \frac{(N-j) !(j-1) !}{(2 N-1) !}\left(2 N-j-\frac{1}{2}\right) \cdots\left(N-j+\frac{1}{2}\right) m_{\left(j, I_{l}, K\right)} .
$$

The assertion is equivalent to the identity

$$
\begin{aligned}
-2^{-2 j} \frac{N !}{(2 N-2 j-1) ! j !} & \left(2 N-j-\frac{1}{2}\right) \cdots\left(N+\frac{1}{2}\right) \frac{1}{j+I_{l}} m_{\left(I_{l}, K\right)} \\
= & \frac{(N-j) !(j-1) !}{(2 N-1) !}\left(2 N-j-\frac{1}{2}\right) \cdots\left(N-j+\frac{1}{2}\right) m_{\left(j, I_{l}, K\right)} .
\end{aligned}
$$

But by the definition of the multiplicities $m_{I}$,

$$
m_{\left(j, I_{l}, K\right)}=-\frac{1}{j+I_{l}} \frac{N !(N-1) !}{j !(j-1) !(N-j) !(N-j-1) !} m_{\left(I_{l}, K\right)} .
$$

The latter relation shows that the assertion is equivalent to 


$$
\begin{aligned}
2^{-2 j} \frac{1}{(2 N-2 j-1) !}\left(2 N-j-\frac{1}{2}\right) \cdots\left(N+\frac{1}{2}\right) \\
\quad=\frac{(N-1) !}{(2 N-1) !}\left(2 N-j-\frac{1}{2}\right) \cdots\left(N-j+\frac{1}{2}\right) \frac{1}{(N-j-1) !} .
\end{aligned}
$$

But this identity is equivalent to (5.3). This completes the proof for non-trivial $K$. The proof immediately extends to trivial $K$.

Next, we prove that the contributions of

$$
P_{2 j} \bar{P}_{2 J}, \quad j+|J|=N
$$

on both sides of (5.1) coincide. On the one hand, (4.24) shows that $P_{2 j} \bar{P}_{2 J}$ contributes to

with the coefficient

$$
D_{2 N}\left(-\frac{n}{2}+2 N-j\right)
$$

$$
-(-1)^{N} 2^{2 N-1} \frac{N !(N-1) !}{(2 N-1) !} \frac{\pi_{2 N}^{\prime}(N-j)}{\left(2 N-j-J_{r}+\frac{1}{2}\right)} \frac{1}{j !(j-1) !(N-j) !(N-j-1) !} m_{J} .
$$

By (5.4), the latter term equals

$$
\begin{aligned}
-(-1)^{N} 2^{2 N-1} \frac{N !(N-1) !}{(2 N-1) !} \frac{1}{j !(N-j-1) !} \\
\times \frac{1}{\left(2 N-j-J_{r}+\frac{1}{2}\right)}\left(2 N-j-\frac{1}{2}\right) \cdots\left(N-j+\frac{1}{2}\right) m_{J} .
\end{aligned}
$$

We compare this coefficient with the coefficient of the contribution of $\bar{P}_{2 J}$ to

$$
D_{2 N-2 j}\left(-\frac{n}{2}+2 N-j\right) \text {. }
$$

For this purpose, we write $J=\left(K, J_{r}\right)$ with a possibly trivial $K$. Let $K$ be non-trivial. Then $|K|=N-J_{r}-j$. Now by (4.19), the composition $\bar{P}_{2 K} \bar{P}_{2 J_{r}}$ contributes with the coefficient

i.e., with

$$
(-1)^{N-j} \frac{2^{2 N-2 j-1}}{(2 N-2 j-1) !} \frac{\pi_{2 N-2 j}\left(\lambda+\frac{n}{2}-(N-j)\right)}{\left(\lambda+\frac{n+1}{2}-(N-j)+|K|\right)} m_{J},
$$

By (5.5), it equals

$$
(-1)^{N-j} \frac{2^{2 N-2 j-1}}{(2 N-2 j-1) !} \frac{\pi_{2 N-2 j}(N)}{\left(2 N-j-J_{r}+\frac{1}{2}\right)} m_{J} .
$$

$$
(-1)^{N-j} \frac{2^{2 N-2 j-1}}{(2 N-2 j-1) !} \frac{N !}{j !} \frac{1}{\left(2 N-j-J_{r}+\frac{1}{2}\right)}\left(2 N-j-\frac{1}{2}\right) \cdots\left(N+\frac{1}{2}\right) m_{J} .
$$

It is straightforward to verify that the coincidence of (5.9) and (5.10) is equivalent to (5.3). This completes the proof for non-trivial $K$. The proof immediately extends to trivial $K$.

Next, we prove that the contributions of

$$
P_{2 I} \bar{P}_{2 j}, \quad|I|+j=N
$$


on both sides of (5.2) coincide. On the one hand, we determine the coefficient of the contribution of $P_{2 I}$ to

$$
D_{2 N-2 j}\left(-\frac{n+1}{2}-j\right)
$$

For this purpose, we write $I=\left(I_{l}, K\right)$ with a possibly trivial $K$. Let $K$ be non-trivial. By (4.14), $P_{2 I}$ contributes with the coefficient

$$
\frac{\pi_{2 N-2 j}\left(\lambda+\frac{n}{2}-(N-j)\right)}{\left(\lambda+\frac{n}{2}-(N-j)-|K|\right)} m_{I}^{(1)} .
$$

Using $|K|=N-j-I_{l}$ and (5.7), the latter expression simplifies to

$$
(-1)^{N-j} \frac{2^{2 N-2 j-1}}{(2 N-2 j-1) !} \frac{N !}{j !} \frac{1}{\left(2 N+\frac{1}{2}-j-I_{l}\right)}\left(2 N-j-\frac{1}{2}\right) \cdots\left(N+\frac{1}{2}\right) m_{I} .
$$

On the other hand, we determine the coefficient of the contribution of the operator $P_{2 I} \bar{P}_{2 j}$ to

$$
D_{2 N}\left(-\frac{n+1}{2}+j\right)
$$

Eq. (4.24) yields

$$
-(-1)^{N} 2^{2 N-1} \frac{N !(N-1) !}{(2 N-1) !} \frac{\pi_{2 N}^{\prime}\left(-\frac{1}{2}+j-N\right)}{\left(-\frac{1}{2}+j-2 N+I_{l}\right)} \frac{1}{(N-j) !(N-j-1) ! j !(j-1) !} m_{I} .
$$

By (5.6), the latter formula simplifies to

$$
(-1)^{N-j} 2^{2 N-1} \frac{N !(N-1) !}{(2 N-1) !} \frac{\left(2 N-j-\frac{1}{2}\right) \cdots\left(N-j+\frac{1}{2}\right)}{\left(2 N+\frac{1}{2}-j+I_{l}\right)(N-j-1) ! j !} m_{I} .
$$

It is straightforward to verify that the equality of (5.11) and (5.12) is equivalent to the relation (5.3). This completes the proof for non-trivial $K$. The proof immediately extends to trivial $K$.

Next, we prove that the contributions of

$$
\bar{P}_{2 J} \bar{P}_{2 j}, \quad|J|+j=N
$$

on both sides of (5.2) coincide. On the one hand, Eq. (4.19) shows that these terms contribute to

$$
D_{2 N}\left(-\frac{n+1}{2}+j\right)
$$

with the coefficient

$$
\pi_{2 N}^{\prime}\left(-\frac{1}{2}+j-N\right) m_{(J, j)}^{(1)}
$$

i.e., with

$$
(-1)^{j}(N-j) !(j-1) !\left(2 N-j-\frac{1}{2}\right) \cdots\left(N-j+\frac{1}{2}\right) m_{(J, j)}^{(1)} .
$$

We compare this coefficient with the coefficient of the contribution of $\bar{P}_{2 J}$ to

$$
D_{2 N-2 j}\left(-\frac{n+1}{2}-j\right) \text {. }
$$


For this purpose, we write $J$ in the form $\left(K, J_{r}\right)$ with a possibly trivial $K$. Let $K$ be non-trivial. Now by (4.19) $\bar{P}_{2 K} \bar{P}_{2 J_{r}}$ contributes with the coefficient

$$
-\frac{\pi_{2 N-2 j}\left(\lambda+\frac{n}{2}-(N-j)\right)}{\lambda+\frac{n}{2}-(N-j)+|K|+\frac{1}{2}} m_{\left(K, J_{r}\right)}^{(1)},
$$

i.e., with

$$
-\frac{\pi_{2 N-2 j}\left(-N-\frac{1}{2}\right)}{J_{r}+j} m_{\left(K, J_{r}\right)}^{(1)} .
$$

Now we claim that the latter coefficient coincides with (5.13) for $J=\left(K, J_{r}\right)$, i.e., with

$$
(-1)^{j}(N-j) !(j-1) !\left(2 N-j-\frac{1}{2}\right) \cdots\left(N-j+\frac{1}{2}\right) m_{\left(K, J_{r}, j\right)}^{(1)} .
$$

By (5.7), the assertion is equivalent to the identity

$$
\begin{aligned}
-\frac{N !}{\left(J_{r}+j\right) j !}\left(N+\frac{1}{2}\right) & \cdots\left(2 N-j-\frac{1}{2}\right) m_{\left(K, J_{r}\right)}^{(1)} \\
& =(-1)^{j}(N-j) !(j-1) !\left(2 N-j-\frac{1}{2}\right) \cdots\left(N-j+\frac{1}{2}\right) m_{\left(K, J_{r}, j\right)}^{(1)} .
\end{aligned}
$$

But

$$
m_{\left(K, J_{r}, j\right)}^{(1)}=-(-1)^{j} \frac{1}{J_{r}+j} \frac{N !(N-1) !}{j !(j-1) !(N-j) !(N-j-1) !} 2^{2 j} \frac{(2 N-2 j-1) !}{(2 N-1) !} m_{\left(K, J_{r}\right)}^{(1)}
$$

shows that the latter identity is equivalent to (5.3). This completes the proof for non-trivial $K$. The proof immediately extends to trivial $K$.

Next, we prove that all contributions of

$$
P_{2 I} \text { with } I_{l} \neq j, \quad P_{2 I} \bar{P}_{2 J} \text { with } I_{l} \neq j \quad \text { and } \quad \bar{P}_{2 J}
$$

to

vanish. Let

$$
D_{2 N}\left(-\frac{n}{2}+2 N-j\right), j=1, \ldots, N
$$

We recall that

$$
\lambda=-\frac{n}{2}+2 N-j .
$$

$$
\pi_{2 N}\left(\lambda+\frac{n}{2}-N\right)=\pi_{2 N}(N-j)=0 \quad \text { for } j=1, \ldots, N .
$$

We first consider $P_{2 I}$. We write $I=\left(I_{l}, J\right)$ with a possibly trivial $J$. Let $J$ be non-trivial. Then we have

$$
\lambda+\frac{n}{2}-N-|J|=J_{l}-j \neq 0,
$$

and the relations (4.14) and (5.16) show that the contribution of $P_{2 I}$ vanishes. It remains to prove that $P_{2 N}$ does not contribute for $j \neq N$. But by (4.11), the corresponding multiplicity is a multiple of

$$
\eta_{2 N}\left(\lambda+\frac{n}{2}-N\right)=\eta_{2 N}(N-j)=0 .
$$


Next, we consider the mixed contributions. Then we have

$$
\lambda+\frac{n}{2}-2 N+I_{l}=I_{l}-j \neq 0
$$

and the relations (4.24) and (5.16) show that the contribution of $P_{2 I} \bar{P}_{2 J}$ vanishes.

Finally, we write $J=\left(K, J_{r}\right)$ with a possibly trivial $K$. Then we have

$$
\lambda+\frac{n+1}{2}-N+|K|=\frac{1}{2}-j+2 N-J_{r} \neq 0,
$$

and the relations (4.19) and (5.16) show that the contribution of $\bar{P}_{2 J}$ vanishes.

Finally, we prove that all contributions of

$$
P_{2 I}, \quad P_{2 I} \bar{P}_{2 J} \text { with } J_{r} \neq j \quad \text { and } \quad \bar{P}_{2 J} \text { with } J_{r} \neq j
$$

to

$$
D_{2 N}\left(-\frac{n+1}{2}+j\right)
$$

vanish. Let

$$
\lambda=-\frac{n+1}{2}+j .
$$

We recall that

$$
\pi_{2 N}\left(\lambda+\frac{n}{2}-N\right)=\pi_{2 N}\left(j-N-\frac{1}{2}\right)=0 \quad \text { for } j=1, \ldots, N .
$$

We first consider $P_{2 I}$. We write $I=\left(I_{l}, J\right)$ with a possibly trivial $J$. Let $J$ be non-trivial. Then we have

$$
\lambda+\frac{n}{2}-N-\left(N-I_{l}\right)=-\frac{1}{2}+j-2 N+I_{l} \neq 0,
$$

and the relations (4.14) and (5.17) show that the contribution of $P_{2 I}$ vanishes.

Next, we consider the mixed contributions. Then we have

$$
\lambda+\frac{n+1}{2}-J_{r}=j-J_{r} \neq 0,
$$

and the relations (4.24) and (5.17) show that the contribution $P_{2 I} \bar{P}_{2 J}$ vanishes.

Finally, we write $J=\left(K, J_{r}\right)$ with a possibly trivial $K$. Then we have

$$
\lambda+\frac{n+1}{2}-N+|K|=j-J_{r} \neq 0
$$

and the relations (4.19) and (5.17) show that the contribution $\bar{P}_{2 J}$ vanishes.

\section{The RESTRICTION PROPERTY AND THE COMMUTATOR RELATIONS}

In the present section, we derive two consequences of Theorem 4.1 which will play a central role in what follows 6

\footnotetext{
${ }^{6}$ In even dimensions and for general metrics the usual restriction $2 N \leq n$ will be in place without mentioning it. Moreover, for locally conformally flat metrics the statements extend to all $N \geq 1$.
} 
Theorem 6.1. For $N \geq 1$, let

$$
D_{2 N}^{r e s}(\lambda)=A_{2 N}\left(\lambda+\frac{n}{2}-N\right)^{2 N-1}+B_{2 N}\left(\lambda+\frac{n}{2}-N\right)^{2 N-2}+\cdots+P_{2 N} .
$$

Then the leading coefficient $A_{2 N}$ is given by the formula

$$
(-1)^{N-1} 2^{-(2 N-1)}(2 N-1) ! A_{2 N}=\mathcal{M}_{2 N}-\overline{\mathcal{M}}_{2 N} .
$$

Moreover, the coefficient

$$
(-1)^{N-1} 2^{-(2 N-2)}(2 N-1) ! B_{2 N}
$$

coincides with the sum of

$$
\mathcal{M}_{2 N}+(N-1)\left(\mathcal{M}_{2 N}-\overline{\mathcal{M}}_{2 N}\right)
$$

and

$$
2 \sum_{a=1}^{N-1} a\left(\begin{array}{c}
N-1 \\
a
\end{array}\right)^{2}\left[\left(\mathcal{M}_{2 N-2 a}-\overline{\mathcal{M}}_{2 N-2 a}\right) \overline{\mathcal{M}}_{2 a}-\mathcal{M}_{2 a}\left(\mathcal{M}_{2 N-2 a}-\overline{\mathcal{M}}_{2 N-2 a}\right)\right] .
$$

Here we used the convention to simplify formulas by omitting the restriction operators $i^{*}$.

Proof. The formula for $A_{2 N}$ follows from the definitions in Sections 4.14.2. In order to prove the assertion for the sub-leading coefficient, we first make that coefficient explicit by using the corresponding formulas in Sections 4.1 4.3, For the pure P-terms we find the formula

$$
\begin{aligned}
m_{I}^{(2)} P_{2 I} & =\left(\left(N-I_{l}\right) \eta_{2 N}^{(2 N-1)}+\eta_{2 N}^{(2 N-2)}\right) m_{I}^{(1)} P_{2 I} \\
& =(-1)^{N-1} \frac{2^{2 N-1}}{(2 N-1) !}\left(\left(N-I_{l}\right)+\frac{N}{2}\right) m_{I} P_{2 I} \\
& =(-1)^{N-1} \frac{2^{2 N-2}}{(2 N-1) !}\left(3 N-2 I_{l}\right) m_{I} P_{2 I}
\end{aligned}
$$

using

$$
\eta_{2 N}^{(2 N-1)}=1 \quad \text { and } \quad \eta_{2 N}^{(2 N-2)}=\frac{N}{2} .
$$

Similarly, for the pure $\bar{P}$-terms we find

$$
\begin{aligned}
m_{\bar{J}}^{(2)} \bar{P}_{2 J} & =\left(\left(-\left(N-J_{r}\right)-\frac{1}{2}\right) \tau_{2 N}^{(2 N-1)}+\tau_{2 N}^{(2 N-2)}+\frac{1}{2} \tau_{2 N}^{(2 N-1)}\right) m_{\bar{J}}^{(1)} \bar{P}_{2 J} \\
& =(-1)^{N-1} \frac{2^{2 N-2}}{(2 N-1) !}\left(N-2 J_{r}+1\right) m_{J} \bar{P}_{2 J}
\end{aligned}
$$

using

$$
\tau_{2 N}^{(2 N-1)}=1 \text { and } \tau_{2 N}^{(2 N-2)}=\frac{N-1}{2} .
$$

Finally, for the mixed terms, (4.23) states

$$
m_{(I, \bar{J})}^{(2)}=(-1)^{N-1} 2^{2 N-1} \frac{N !(N-1) !}{(2 N-1) !} \frac{m_{I} m_{J}}{|I| !(|I|-1) !|J| !(|J|-1) !} .
$$


Now we prove that these contributions coincide with those given in the theorem. In fact, for the pure $P$-terms and the pure $\bar{P}$-terms, the assertions are

$$
N m_{I}-2 \sum_{a=1}^{N-1} a\left(\begin{array}{c}
N-1 \\
a
\end{array}\right)^{2}\left[P_{2 I}: \mathcal{M}_{2 a} \mathcal{M}_{2 N-2 a}\right]=\left(3 N-2 I_{l}\right) m_{I}
$$

and

$$
-(N-1) m_{J}-2 \sum_{a=1}^{N-1} a\left(\begin{array}{c}
N-1 \\
a
\end{array}\right)^{2}\left[\bar{P}_{2 J}: \overline{\mathcal{M}}_{2 N-2 a} \overline{\mathcal{M}}_{2 a}\right]=\left(N-2 J_{r}+1\right) m_{J} .
$$

Moreover, for the mixed terms the assertion is

$$
\begin{aligned}
\sum_{a=1}^{N-1} a\left(\begin{array}{c}
N-1 \\
a
\end{array}\right)^{2}\left[P_{2 I} \bar{P}_{2 J}:\left(\mathcal{M}_{2 N-2 a} \overline{\mathcal{M}}_{2 a}+\mathcal{M}_{2 a} \overline{\mathcal{M}}_{2 N-2 a}\right)\right] \\
=\frac{N !(N-1) !}{|I| !(|I|-1) !|J| !(|J|-1) !} m_{I} m_{J}
\end{aligned}
$$

i.e.,

$$
|J|\left(\begin{array}{c}
N-1 \\
|J|
\end{array}\right)^{2}+|I|\left(\begin{array}{c}
N-1 \\
|I|
\end{array}\right)^{2}=\frac{N !(N-1) !}{|I| !(|I|-1) !|J| !(|J|-1) !} .
$$

The latter relation easily follows from $|I|+|J|=N$. The remaining relations (6.5) and (6.6) are consequences of the following conformal variational formula.

Theorem 6.2 ([J09b]). On manifolds $M$ of dimension $n$, we have

$$
\begin{aligned}
-\left.(d / d t)\right|_{0}\left(e^{\left(\frac{n}{2}+N\right) t \varphi} \mathcal{M}_{2 N}\left(e^{2 t \varphi} g\right)\right. & \left.e^{-\left(\frac{n}{2}-N\right) t \varphi}\right) \\
& =\sum_{a=1}^{N-1} a\left(\begin{array}{c}
N-1 \\
a
\end{array}\right)^{2}\left[\mathcal{M}_{2 N-2 a}(g),\left[\mathcal{M}_{2 a}(g), \varphi\right]\right]
\end{aligned}
$$

for all $\varphi \in C^{\infty}(M)$. On the right-hand side of (6.7), $\varphi$ is regarded as a multiplication operator.

In fact, by the conformal covariance of the GJMS-operators, one finds that the equality of the respective contributions of the term $\varphi P_{2 I}$ for the composition $I=$ $\left(I_{1}, \ldots, I_{r}\right)$ to both sides of (6.7) is equivalent to the relation

$$
\begin{aligned}
-\left(N-I_{1}\right) m_{I}= & \left(\begin{array}{c}
N-1 \\
I_{1}
\end{array}\right)^{2} I_{1} m_{\left(I_{1}\right)} m_{\left(I_{2}, I_{2}, \ldots, I_{r}\right)} \\
& +\cdots+\left(\begin{array}{c}
N-1 \\
I_{1}+\cdots+I_{r-1}
\end{array}\right)^{2}\left(I_{1}+\cdots+I_{r-1}\right) m_{\left(I_{1}, \ldots, I_{r-1}\right)} m_{\left(I_{r}\right)} .
\end{aligned}
$$

Similarly, the equality of the respective contributions of the term $P_{2 J} \varphi$ for the composition $J=\left(J_{1}, \ldots, J_{r}\right)$ to both sides of (6.7) is equivalent to the relation

$$
\begin{aligned}
-\left(N-J_{r}\right) m_{J}=\left(\begin{array}{c}
N-1 \\
J_{2}+\cdots+J_{r}
\end{array}\right)^{2}\left(J_{2}+\cdots+J_{r}\right) m_{\left(J_{1}\right)} m_{\left(J_{2}, \ldots, J_{r}\right)} \\
+\cdots+\left(\begin{array}{c}
N-1 \\
J_{r}
\end{array}\right)^{2} J_{r} m_{\left(J_{1}, J_{2}, \ldots, J_{r-1}\right)} m_{\left(J_{r}\right)} .
\end{aligned}
$$


In order to prove (6.8), we use the explicit formula (2.1) for the coefficients $m_{I}$ to write the terms on the right-hand side as multiples of $m_{I}$. We find

$$
\begin{aligned}
-\frac{1}{N}\left[\left(I_{2}+\cdots+I_{r}\right)\left(I_{1}+I_{2}\right)+\left(I_{3}+\cdots+I_{r}\right)\left(I_{2}\right.\right. & \left.+I_{3}\right) \\
& \left.+\cdots+I_{r}\left(I_{r-1}+I_{r}\right)\right] m_{I} .
\end{aligned}
$$

Now the relation

$$
\left(I_{2}+\cdots+I_{r}\right)\left(I_{1}+I_{2}\right)+\cdots+I_{r}\left(I_{r-1}+I_{r}\right)=\left(I_{1}+\cdots+I_{r}\right)\left(I_{2}+\cdots+I_{r}\right)
$$

(which follows by induction on the number of entries) implies that in (6.10) the sum in parentheses equals $N\left(N-I_{1}\right)$ if $|I|=N$. Thus, (6.10) equals $-\left(N-I_{1}\right) m_{I}$. This proves the assertion and (6.5).

Similarly, the identity (6.9) follows by applying (6.8) to the inverse composition $I^{-1}$ of $I$ using the relations $m_{I^{-1}}=m_{I}$. This proves (6.6).

As a consequence of Theorem 6.1, we obtain the restriction property of $\mathcal{M}_{2 N}$.

Theorem 6.3 (Restriction property). For $N \geq 2$, we have

$$
\mathcal{M}_{2 N} i^{*}=i^{*} \overline{\mathcal{M}}_{2 N} \text {. }
$$

The special case $\mathcal{M}_{4} i^{*}=i^{*} \overline{\mathcal{M}}_{4}$ of the restriction property (6.11) was found and applied in J09a (see Lemma 6.11.6).

Proof. We recall that the degree of the polynomial $\lambda \mapsto D_{2 N}^{r e s}(\lambda)$ is $N$. But since $N<2 N-1$ for $N \geq 2$, the coefficient $A_{2 N}$ vanishes. Thus, the assertion follows from (6.1).

Similarly, for $N \geq 3$, the sub-leading coefficient of the degree $N$ polynomial $D_{2 N}^{r e s}(\lambda)$ vanishes. Hence the second part of Theorem 6.1 and Theorem 6.3 imply that

$$
\mathcal{M}_{2 N}+(2 N-2)\left(\left(\mathcal{M}_{2}-\overline{\mathcal{M}}_{2}\right) \overline{\mathcal{M}}_{2 N-2}-\mathcal{M}_{2 N-2}\left(\mathcal{M}_{2}-\overline{\mathcal{M}}_{2}\right)\right)=0 .
$$

Now adding a multiple of the relation

$$
\mathcal{M}_{2}\left(\mathcal{M}_{2 N-2}-\overline{\mathcal{M}}_{2 N-2}\right)-\left(\mathcal{M}_{2 N-2}-\overline{\mathcal{M}}_{2 N-2}\right) \overline{\mathcal{M}}_{2}=0
$$

cancels the mixed terms $\mathcal{M}_{2} \overline{\mathcal{M}}_{2 N-2}$ and $\mathcal{M}_{2 N-2} \overline{\mathcal{M}}_{2}$, and proves

Theorem 6.4 (Commutator relations). For $N \geq 3$, the commutator relations

$$
\mathcal{M}_{2 N} i^{*}=(2 N-2)\left(i^{*}\left[\overline{\mathcal{M}}_{2}, \overline{\mathcal{M}}_{2 N-2}\right]-\left[\mathcal{M}_{2}, \mathcal{M}_{2 N-2}\right] i^{*}\right)
$$

hold true.

The special case $N=3$ of the commutator relations (6.12) already played an important role in [J09a] (see Theorem 6.11.17).

We also make the lowest-order cases of Theorem 6.1 fully explicit.

Example 6.1. $-3 ! A_{4}=8\left(\mathcal{M}_{4}-\overline{\mathcal{M}}_{4}\right)$ and

$$
-\frac{3 !}{4} B_{4}=\mathcal{M}_{4}+\left(\mathcal{M}_{4}-\overline{\mathcal{M}}_{4}\right)+2\left[\left(\mathcal{M}_{2}-\overline{\mathcal{M}}_{2}\right) \overline{\mathcal{M}}_{2}-\mathcal{M}_{2}\left(\mathcal{M}_{2}-\overline{\mathcal{M}}_{2}\right)\right] .
$$


Example 6.2. $5 ! A_{6}=32\left(\mathcal{M}_{6}-\overline{\mathcal{M}}_{6}\right)$ and

$$
\begin{aligned}
\frac{5 !}{16} B_{6} & =\mathcal{M}_{6}+2\left(\mathcal{M}_{6}-\overline{\mathcal{M}}_{6}\right) \\
& +8\left[\left(\mathcal{M}_{4}-\overline{\mathcal{M}}_{4}\right) \overline{\mathcal{M}}_{2}-\mathcal{M}_{2}\left(\mathcal{M}_{4}-\overline{\mathcal{M}}_{4}\right)\right] \\
& +4\left[\left(\mathcal{M}_{2}-\overline{\mathcal{M}}_{2}\right) \overline{\mathcal{M}}_{4}-\mathcal{M}_{4}\left(\mathcal{M}_{2}-\overline{\mathcal{M}}_{2}\right)\right]
\end{aligned}
$$

In particular, the restriction properties $\mathcal{M}_{2 N}=\overline{\mathcal{M}}_{2 N}$ for $N=2,3$ imply the commutator relation for $\mathcal{M}_{6}$.

Example 6.3. $-7 ! A_{8}=128\left(\mathcal{M}_{8}-\overline{\mathcal{M}}_{8}\right)$ and

$$
\begin{aligned}
-\frac{7 !}{64} B_{8} & =\mathcal{M}_{8}+3\left(\mathcal{M}_{8}-\overline{\mathcal{M}}_{8}\right) \\
& +18\left[\left(\mathcal{M}_{6}-\overline{\mathcal{M}}_{6}\right) \overline{\mathcal{M}}_{2}-\mathcal{M}_{2}\left(\mathcal{M}_{6}-\overline{\mathcal{M}}_{6}\right)\right] \\
& +36\left[\left(\mathcal{M}_{4}-\overline{\mathcal{M}}_{4}\right) \overline{\mathcal{M}}_{4}-\mathcal{M}_{4}\left(\mathcal{M}_{4}-\overline{\mathcal{M}}_{4}\right)\right] \\
& +6\left[\left(\mathcal{M}_{2}-\overline{\mathcal{M}}_{2}\right) \overline{\mathcal{M}}_{6}-\mathcal{M}_{6}\left(\mathcal{M}_{2}-\overline{\mathcal{M}}_{2}\right)\right]
\end{aligned}
$$

In particular, the restriction properties $\mathcal{M}_{2 N}=\overline{\mathcal{M}}_{2 N}$ for $N=2,3,4$ imply the commutator relation for $\mathcal{M}_{8}$.

It is natural and will be convenient later on to reformulate the commutator relations (6.12) in terms of generating functions. Let

$$
\mathcal{H}(s) \text { and } \overline{\mathcal{H}}(s)
$$

be the respective generating functions (1.7) of the sequences

$$
\mathcal{M}_{2}, \mathcal{M}_{4}, \cdots \text { and } \overline{\mathcal{M}}_{2}, \overline{\mathcal{M}}_{4}, \cdots
$$

Then the system of relations (6.12) is equivalent to the identity

$$
\frac{1}{s} \frac{\partial \mathcal{H}}{\partial s}(s) i^{*}=\frac{1}{2} \mathcal{M}_{4} i^{*}+i^{*}\left[\bar{P}_{2}, \overline{\mathcal{H}}(s)\right]-\left[P_{2}, \mathcal{H}(s)\right] i^{*} .
$$

Indeed, the equivalence of (6.12) and (6.13) follows from the calculation

$$
\begin{aligned}
& \frac{1}{s} \frac{\partial \mathcal{H}}{\partial s}(s) i^{*}=\frac{1}{2} \sum_{N \geq 2} \mathcal{M}_{2 N} i^{*} \frac{1}{(N-2) !(N-1) !}\left(\frac{s^{2}}{4}\right)^{N-2} \\
& =\frac{1}{2} \mathcal{M}_{4} i^{*}+\sum_{N \geq 3}\left(i^{*}\left[\bar{P}_{2}, \overline{\mathcal{M}}_{2 N-2}\right]-\left[P_{2}, \mathcal{M}_{2 N-2}\right] i^{*}\right) \frac{(N-1)}{(N-2) !(N-1) !}\left(\frac{s^{2}}{4}\right)^{N-2} \\
& =\frac{1}{2} \mathcal{M}_{4} i^{*}+\left(i^{*}\left[\bar{P}_{2}, \overline{\mathcal{H}}(s)\right]-\left[P_{2}, \mathcal{H}(s)\right] i^{*}\right) .
\end{aligned}
$$

A first consequence of the commutator relations (6.12) is that the operators $\mathcal{M}_{2 N}$ are only of order two. This is obvious for $\mathcal{M}_{2}$ and easy to see for $\mathcal{M}_{4}$. The general case follows by induction. In fact, if we assume that for any metric $\mathcal{M}_{2 N}$ is a secondorder operator, then $\overline{\mathcal{M}}_{2 N}$ is second-order, too. It follows that the commutators on the right-hand side of the relation

$$
\mathcal{M}_{2 N+2} i^{*}=2 N\left(i^{*}\left[\bar{P}_{2}, \overline{\mathcal{M}}_{2 N}\right]-\left[P_{2}, \mathcal{M}_{2 N}\right] i^{*}\right)
$$


are of third order. Moreover, this relation also states that its right-hand side is a tangential operator. But, by the self-adjointness of $\mathcal{M}_{2 N+2}$, this tangential operator is only of order two. This completes the induction. In the following section, we shall substantially refine this argument.

\section{The PRincipal Part of $\mathcal{M}_{2 N}$}

In this section we prove the formula for the principal part of the operators $\mathcal{M}_{2 N}$ stated in Theorem 1.1. The main tool in the proof will be Theorem 6.4.

7.1. Description of the principal part. The following result restates a part of Theorem 1.1

Theorem 7.1. On any Riemannian manifold $(M, g)$ of dimension $n \geq 3$,

$$
\sum_{N \geq 1} \mathcal{M}_{2 N}^{0}(g) \frac{1}{(N-1) !^{2}}\left(\frac{r^{2}}{4}\right)^{N-1}=-\delta\left(g_{r}^{-1} d\right) .
$$

Some comments are in order.

Here we use the following notational conventions. We define $L^{0}(u) \stackrel{\text { def }}{=} L(u)-L(1) u$ for any linear operator $L$ on $C^{\infty}(M)$, i.e., $L^{0}$ is obtained by removing the zeroth-order term $L(1)$ of $L$. We regard $g_{r}$ as an endomorphism on one-forms on $M$ using $g$, and denote the adjoint of $d$ (negative divergence) with respect to $g$ by $\delta$. Moreover, we recall that according to the conventions in Section 1, in even dimension $n$ and for general metrics, the sum in (7.1) only runs up to $r^{n-2}$.

For a few special metrics, Theorem 7.1 can be given a direct proof. In fact, for round spheres $\mathbb{S}^{n}$ and even for their conformally flat pseudo-Riemannian analogs $\mathbb{S}^{(p, q)}$, the assertion follows from summation formulas established in [J09b] and [JK09]. These proofs utilize the fact that, by the relation to representation theory, explicit formulas for GJMS-operators are available in these cases.

In the low-order cases $N=1,2,3$, Theorem 7.1 for general metrics can be confirmed by explicit formulas. In fact, the relation $\mathcal{M}_{2}^{0}=-\delta d=\Delta$ is obvious, and 7

$$
\frac{1}{4} \mathcal{M}_{4}^{0}=-\delta\left(\left(g_{r}\right)_{(2)}^{-1} d\right)
$$

is equivalent to

$$
\mathcal{M}_{4}^{0}=-4 \delta(\mathrm{Pd})
$$

which follows from (1.1) and (1.4) by a direct calculation. Finally, for $n \geq 6$, we have (by Section 6.12 of [J09a]) the explicit formula

$$
\mathcal{M}_{6}^{0}=-48 \delta\left(\mathrm{P}^{2} d\right)-\frac{16}{n-4} \delta(\mathcal{B} d)
$$

(for more details see also Section 10.1.1). But

$$
g_{r}=g-\mathrm{Pr}^{2}+\frac{1}{4}\left(\mathrm{P}^{2}-\frac{\mathcal{B}}{n-4}\right) r^{4}+\cdots
$$

\footnotetext{
${ }^{7}$ The subscript $(2 N)$ indicates the coefficient of $r^{2 N}$ in the Taylor expansion.
} 
implies

$$
g_{r}^{-1}=g+\mathrm{Pr}^{2}+\frac{1}{4}\left(3 \mathrm{P}^{2}+\frac{\mathcal{B}}{n-4}\right) r^{4}+\cdots,
$$

and the above formula for $\mathcal{M}_{6}$ can be written in the form

$$
\mathcal{M}_{6}^{0}=-2 ! 2 ! 2^{4} \delta\left(\left(g_{r}^{-1}\right)_{(4)} d\right) .
$$

Theorem 7.1 confirms Conjecture 11.1 in $\mathrm{J} 09 \mathrm{~b}]$.

7.2. Proof of Theorem 7.1. In this section, we prove that the commutator relations (6.12) imply the precise form of the principal part of $\mathcal{M}_{2 N}$ claimed in Theorem 7.1.

As a preparation, we establish a result which might be also of independent interest.

Theorem 7.2. Let $\bar{g}(r)=d r^{2}+g(r)$ (with $g_{r}=g(r)$ ). Then the metric

$$
g_{++}=s^{-2}\left(d s^{2}+g(r, s)\right)=s^{-2}\left(d s^{2}+d r^{2}+\sum_{k \geq 0}\left(s^{2}+r^{2}\right)^{k} g_{(2 k)}\right)
$$

satisfies

$$
\operatorname{Ric}\left(g_{++}\right)+(n+1) g_{++}=0 \quad \text { and } \quad g(r, 0)=\bar{g}(r),
$$

i.e., is a Poincaré-Einstein metric relative to $\bar{g}(r)$.

Proof. We calculate the Ricci curvature of (7.3). For this purpose, we use polar coordinates $r=R \cos \theta$ and $s=R \sin \theta$ and write the metric in the form

$$
g_{++}=\sin ^{-2} \theta\left(R^{-2}\left(d R^{2}+\sum_{k \geq 0} R^{2 k} g_{(2 k)}\right)+d \theta^{2}\right)=\sin ^{-2} \theta\left(g_{+}+d \theta^{2}\right)
$$

on $M \times(0, \varepsilon) \times(0, \pi)$. We recall the conformal transformation law

$$
\begin{array}{r}
\operatorname{Ric}(\hat{g})(X, Y)=\operatorname{Ric}(g)(X, Y)-(n-2) g\left(\nabla_{X}^{g} \operatorname{grad} \varphi, Y\right)-g(X, Y) \Delta_{g}(\varphi) \\
\left.-(n-2)|d \varphi|^{2} g(X, Y)+(n-2)\langle d \varphi, X\rangle\right)\langle d \varphi, Y\rangle,
\end{array}
$$

where $\hat{g}=e^{2 \varphi} g$. For $\varphi=-\log \sin \theta$, we have

$$
d \varphi=-\cot \theta d \theta \text { and } \Delta \varphi=\sin ^{-2} \theta .
$$

Thus, (7.4) and (7.5) imply that on the $n+2$-dimensional space $M \times(0, \varepsilon) \times(0, \pi)$

$$
\begin{aligned}
\operatorname{Ric}\left(g_{++}\right) & =\operatorname{Ric}\left(g_{+}+d \theta^{2}\right)-\sin ^{-2} \theta g_{+}-n \cot ^{2} \theta g_{+} \\
& =\operatorname{Ric}\left(g_{+}\right)-\sin ^{-2} \theta g_{+}-n \cot ^{2} \theta g_{+} \\
& =-n g_{+}-\sin ^{-2} \theta g_{+}-n \cot ^{2} \theta g_{+} \\
& =-(n+1) \sin ^{-2} \theta g_{+} \\
& =-(n+1) g_{++}
\end{aligned}
$$

on tangential vectors with a vanishing $\partial_{\theta}$-component. Similarly, on tangential vectors $X=Y=\partial_{\theta}$, we find

$$
\begin{aligned}
\operatorname{Ric}\left(g_{++}\right) & =-n \partial(-\cot \theta) / \partial \theta-\sin ^{-2} \theta-n \cot ^{2} \theta+n \cot ^{2} \theta \\
& =-(n+1) \sin ^{-2} \theta \\
& =-(n+1) g_{++}
\end{aligned}
$$


The remaining components of $\operatorname{Ric}\left(g_{++}\right)$vanish. Thus, we obtain

$$
\operatorname{Ric}\left(g_{++}\right)+(n+1) g_{++}=0 .
$$

The proof is complete.

Remark 7.1. For odd $n, \bar{g}$ is uniquely determined by $g$ to any order. Although $\bar{g}$ lives on a space of even dimension $n+1$, Theorem 7.2 tells that the Poincaré-Einstein metric $g_{++}$relative to $\bar{g}$ is well-defined to any order 8

As an immediate consequence of Theorem 7.2 , we obtain

Corollary 7.1. The coefficients $g_{(2 k, 2 l)}$ in the Poincaré-Einstein metric

$$
g_{++}=s^{-2}\left(d s^{2}+d r^{2}+\sum_{k, l \geq 0} r^{2 k} s^{2 l} g_{(2 k, 2 l)}\right)
$$

are given by

$$
g_{(2 k, 2 l)}=\left(\begin{array}{c}
k+l \\
k
\end{array}\right) g_{(2 k+2 l)} .
$$

In particular, they satisfy the symmetry relations

$$
g_{(2 k, 2 l)}=g_{(2 l, 2 k)} .
$$

For the following discussion, it will be convenient to introduce some additional notational conventions. We shall write Poincaré-Einstein metrics also in the form $r^{-2}\left(d r^{2}+E(g)(r)\right)$ with $E(g)(0)=g$. This notation enables us to emphasize the dependence on the metric $g$. As usual, we shall view a bilinear form $B$ also as an endomorphism on one-forms. The latter acts on the one-form $\omega=\sum_{i} \omega_{i} d x^{i}$ by

$$
\omega \mapsto \sum_{i, j} B_{j}^{i} \omega_{i} d x^{j},
$$

where $B_{j}^{i}=\sum_{k} g^{i k} B_{k j}$. In particular, $E(g)(r)$ will denote a one-parameter family of metrics with $E(g)(0)=g$ and a one-parameter family of linear operators on one-forms with $E(g)(0)=1$.

Theorem 7.2 immediately yields the following restriction property. For similar results in a more general setting see Section 6 of [Go10].

Lemma 7.1. As families of endomorphisms on one-forms, we have

$$
\left.E\left(d r^{2}+E(g)(r)\right)(s)\right|_{r=0}=\left(\begin{array}{cc}
1 & 0 \\
0 & E(g)(s)
\end{array}\right) .
$$

Proof. By Theorem [7.2, the restriction to $r=0$ of the metric

$$
E\left(d r^{2}+E(g)(r)\right)(s)
$$

is given by

$$
d r^{2}+\sum_{l \geq 0} s^{2 l} g_{(2 l)}
$$

\footnotetext{
${ }^{8} \mathrm{An}$ explicit formula for the diffeomorphism which relates the Poincaré-Einstein metrics relative to $\bar{g}$ and the Einstein metric $r^{-2} \bar{g}$ is given in Section 4 of [GW11].
} 
This shows that the corresponding operator (with respect to the background metric $\left.\left.\left(d r^{2}+E(g)(r)\right)\right|_{r=0}=d r^{2}+g\right)$ equals

$$
\left(\begin{array}{cc}
1 & 0 \\
0 & E(g)(s)
\end{array}\right)
$$

The proof is complete.

Next, we apply Lemma 7.1 to prove the following technical result.

Lemma 7.2. The following identities of endomorphisms on one-forms are equivalent:

$$
\begin{aligned}
\left(\begin{array}{cc}
0 & 0 \\
0 & -s^{-1}(\partial / \partial s)\left(E(g)(s)^{-1}\right)+2 E(g)(s)^{-1} \mathrm{P}
\end{array}\right) \\
\quad=-\left.\left(\partial^{2} / \partial r^{2}\right)\right|_{r=0}\left(E\left(d r^{2}+E(g)(r)\right)(s)^{-1}\right)
\end{aligned}
$$

and

$$
\left(\begin{array}{cc}
0 & 0 \\
0 & s^{-1}(\partial / \partial s)(E(g)(s))+2 \mathrm{P} E(g)(s)
\end{array}\right)=\left.\left(\partial^{2} / \partial r^{2}\right)\right|_{r=0}\left(E\left(d r^{2}+E(g)(r)\right)(s)\right) .
$$

Proof. Composing (7.7) from left and right with $\left(\begin{array}{cc}1 & 0 \\ 0 & E(g)(s)\end{array}\right)$ shows that (7.7) is equivalent to the identity

$$
\begin{aligned}
& \left(\begin{array}{cc}
0 & 0 \\
0 & -s^{-1} E(g)(s) \circ(\partial / \partial s)\left(E(g)(s)^{-1}\right) \circ E(g)(s)+2 \mathrm{P} E(g)(s)
\end{array}\right) \\
& =-\left.\left(\begin{array}{cc}
1 & 0 \\
0 & E(g)(s)
\end{array}\right) \circ\left(\partial^{2} / \partial r^{2}\right)\right|_{r=0}\left(E\left(d r^{2}+E(g)(r)\right)(s)^{-1}\right) \circ\left(\begin{array}{cc}
1 & 0 \\
0 & E(g)(s)
\end{array}\right) .
\end{aligned}
$$

Now $E(g)(s)^{-1} E(g)(s)=1$ implies

$$
(\partial / \partial s)\left(E(g)(s)^{-1}\right)=-E(g)(s)^{-1} \circ(\partial / \partial s)(E(g)(s)) \circ E(g)(s)^{-1} .
$$

Similarly, we have

$$
\begin{aligned}
\left.\left(\partial^{2} / \partial r^{2}\right)\right|_{r=0} & \left.\left(E\left(d r^{2}+E(g)(r)\right)(s)^{-1}\right) \circ E\left(d r^{2}+E(g)(r)\right)(s)\right|_{r=0} \\
& +\left.\left.E\left(d r^{2}+E(g)(r)\right)(s)^{-1}\right|_{r=0} \circ\left(\partial^{2} / \partial r^{2}\right)\right|_{r=0}\left(E\left(d r^{2}+E(g)(r)\right)(s)\right)=0 .
\end{aligned}
$$

Combining these observations with Lemma 7.1 proves the assertion.

Next, we use Theorem 7.2 to establish the identity (7.8).

Lemma 7.3. The identity (7.8) holds true.

Proof. Obviously, it suffices to prove that

$$
\begin{aligned}
& \frac{1}{s} \frac{\partial}{\partial s}\left(g^{i a}\left(\sum_{k \geq 0} s^{2 k} g_{(2 k)}\right)_{a j}\right)+2 \mathrm{P}_{b}^{i} g^{b c}\left(\sum_{k \geq 0} s^{2 k} g_{(2 k)}\right)_{c j} \\
& =\left.\frac{\partial^{2}}{\partial r^{2}}\right|_{r=0}\left(\left(\sum_{k \geq 0} r^{2 k} g_{(2 k)}\right)_{i a}^{-1}\left(\sum_{l, m \geq 0} r^{2 l} s^{2 m} g_{(2 l, 2 m)}\right)_{a j}\right) \text {. }
\end{aligned}
$$


In order to prove (7.9), we compare the coefficients of $s^{2 k-2}$ for $k \geq 1$ on both sides. On the left-hand side, this coefficient is given by

$$
2 k g^{i a}\left(g_{(2 k)}\right)_{a j}+2 \mathrm{P}^{i c}\left(g_{(2 k-2)}\right)_{c j} .
$$

Since the right-hand side of (7.9) equals

$$
\left.\frac{\partial^{2}}{\partial r^{2}}\right|_{r=0}\left(\left(g+r^{2} g_{(2)}+\cdots\right)_{i a}^{-1}\right) \sum_{m \geq 0} s^{2 m}\left(g_{(0,2 m)}\right)_{a j}+2 g^{i a} \sum_{m \geq 0} s^{2 m}\left(g_{(2,2 m)}\right)_{a j},
$$

it follows that $s^{2 k-2}$ contributes with the coefficient

$$
-2 g_{(2)}^{i a}\left(g_{(2 k-2)}\right)_{a j}+2 g^{i a}\left(g_{(2,2 k-2)}\right)_{a j} .
$$

But $g_{(2)}=-\mathrm{P}$ and

$$
\left.g_{(2,2 k-2)}=k g_{(2 k)} \quad \text { (by Theorem } \mathbf{7 . 2}\right)
$$

show that (7.10) and (7.11) coincide.

Now we are ready to prove the following result.

Theorem 7.3. The relation (6.13) implies Theorem 7.1.

Proof. We start by determining the principal part of the right-hand side of (6.13) using Theorem 7.1. In fact, the principal part of the right-hand side of (6.13) coincides with the principal part of

$$
-2 \delta(\mathrm{P} d) s^{0}+i^{*}\left[\Delta_{\bar{g}}, \overline{\mathcal{H}}^{0}(s)\right]-\left[\Delta_{g}, \mathcal{H}^{0}(s)\right] i^{*} .
$$

Now we have

$$
\Delta_{\bar{g}}=\frac{\partial^{2}}{\partial r^{2}}+\frac{1}{2} \operatorname{tr}\left(\frac{\partial g_{r} / \partial r}{g_{r}}\right) \frac{\partial}{\partial r}+\Delta_{g_{r}}
$$

and

$$
\overline{\mathcal{H}}^{0}(s)=-\delta_{d r^{2}+g_{r}}\left(E\left(d r^{2}+E(g)(r)\right)(s)^{-1} d\right)
$$

by Theorem [7.1. Lemma 7.1 shows that

$$
i^{*} \delta_{d r^{2}+g_{r}}\left(E\left(d r^{2}+E(g)(r)\right)(s)^{-1} d\right)=\delta_{g}\left(E(g)(s)^{-1} d\right) i^{*} .
$$

Hence using $i^{*} \Delta_{g_{r}}=\Delta_{g} i^{*}$ it follows that

$$
i^{*}\left[\Delta_{g_{r}}, \overline{\mathcal{H}}^{0}(s)\right]-\left[\Delta_{g}, \mathcal{H}^{0}(s)\right] i^{*}
$$

vanishes. Thus, it suffices to determine the principal part of

$$
i^{*}\left[\left(\partial^{2} / \partial r^{2}\right),-\delta_{d r^{2}+g_{r}}\left(E\left(d r^{2}+E(g)(r)\right)(s)^{-1} d\right)\right] .
$$

Since partial derivatives commute, it can be written in the form

$$
-\delta_{g}(T(s) d)
$$

with

$$
T(s)=\left(\begin{array}{cc}
0 & 0 \\
0 & 2 E(g)(s)^{-1} \mathrm{P}
\end{array}\right)+\left.\left(\partial^{2} / \partial r^{2}\right)\right|_{r=0}\left(E\left(d r^{2}+E(g)(r)\right)(s)^{-1}\right) .
$$

But by Lemma 7.2 and Lemma 7.3 , the operator $T(s)$ coincides with

$$
\left(\begin{array}{cc}
0 & 0 \\
0 & s^{-1}(\partial / \partial s)\left(E(g)(s)^{-1}\right)
\end{array}\right)
$$


Now we use induction on $N$. Let $E_{2 N}(g)(s)^{-1}$ denote the coefficient of $s^{2 N}$ in the Taylor expansion of $E(g)(s)^{-1}$. Assume that the relation

$$
\mathcal{M}_{2 N-2}^{0}(g) \frac{1}{(N-2) !^{2}} \frac{1}{2^{2 N-4}}=-\delta_{g}\left(E_{2 N-4}(g)(s)^{-1} d\right), N \geq 3
$$

has been proved for all metrics $g$. Then the above arguments also show that the principal part of

$$
\left(i^{*}\left[\bar{P}_{2}, \overline{\mathcal{M}}_{2 N-2}\right]-\left[P_{2}, \mathcal{M}_{2 N-2}\right] i^{*}\right) \frac{1}{(N-2) !^{2}}\left(\frac{s^{2}}{4}\right)^{N-2}, N \geq 3
$$

is given by

$$
-(2 N-2) \delta_{g}\left(E_{2 N-2}(g)(s)^{-1} d\right) s^{2 N-4} .
$$

Now (6.12) implies that the operator

$$
\frac{1}{(N-1) !^{2}} \frac{1}{2^{2 N-2}} \mathcal{M}_{2 N}(g)
$$

has the principal part

$$
\begin{aligned}
-\frac{1}{(N-1) !^{2}} \frac{1}{2^{2 N-2}}(2 N-2)(N-2) !^{2} 2^{2 N-4}(2 N-2) \delta_{g}\left(E_{2 N-2}(g)(s)^{-1} d\right) & \\
& =-\delta_{g}\left(E_{2 N-2}(g)(s)^{-1} d\right)
\end{aligned}
$$

This completes the induction.

Finally, we note that for locally conformally flat metrics, the above discussion can be made completely explicit. In fact, in that case, we have

$$
E(s)=\left(1-s^{2} / 2 \mathrm{P}\right)^{2}: \Omega^{1}(M) \rightarrow \Omega^{1}(M)
$$

and

$$
E\left(d r^{2}+E(g)(r)\right)(s)=\left(\begin{array}{cc}
1 & 0 \\
0 & \left(1-s^{2} / 2 \mathrm{P}\left(1-r^{2} / 2 \mathrm{P}\right)^{-1}\right)^{2}
\end{array}\right) .
$$

In order to prove (7.14), we apply (7.13) to the metric $\bar{g}$. In fact, we recall that $\bar{g}$ is locally conformally flat, too. We find

$$
E\left(d r^{2}+E(g)(r)\right)(s)=\left(1-s^{2} / 2 \overline{\mathrm{P}}\right)^{2} .
$$

But the relation

$$
\overline{\mathrm{P}}=\mathrm{P}\left(d r^{2}+g_{r}\right)=-\frac{1}{2 r}(\partial / \partial r)\left(g_{r}\right)
$$

(see [J09a], Lemma 6.11.2 and Appendix 12.5, Lemma 12.1) implies

$$
\overline{\mathrm{P}}=\mathrm{P}\left(1-r^{2} / 2 \mathrm{P}\right)^{-1} \text {. }
$$

This yields (7.14). Now by (7.13), the left-hand side of (7.8) equals

$$
-2 \mathrm{P}\left(1-s^{2} / 2 \mathrm{P}\right)+2 \mathrm{P}\left(1-s^{2} / 2 \mathrm{P}\right)^{2}=-s^{2} \mathrm{P}^{2}\left(1-s^{2} / 2 \mathrm{P}\right) .
$$

But an easy calculation using (7.14) shows that this result coincides with the righthand side of (17.8). 


\section{The ZEROTH-ORDER TERM OF $\mathcal{M}_{2 N}$}

The inversion formula in Theorem 2.1 proves the first part of Theorem 1.1. In the present section, we complete the proof of the second part of Theorem 1.1.

First, we recall the definition (1.7) of the generating function $\mathcal{H}(r)$ and restate the second part of Theorem 1.1 in the following form.

Theorem 8.1. We have,

$$
\mathcal{H}(g)(r)=-\delta\left(g_{r}^{-1} d\right)-\frac{\left(\partial^{2} / \partial r^{2}-(n-1) r^{-1} \partial / \partial r-\delta\left(g_{r}^{-1} d\right)\right)(w(r))}{w(r)} .
$$

The equivalence of the second part of Theorem 1.1 and Theorem 8.1 is a consequence of the following result.

\section{Lemma 8.1.}

$$
\begin{aligned}
\left(\partial^{2} / \partial r^{2}-(n-1) r^{-1} \partial / \partial r-\delta\left(g_{r}^{-1} d\right)\right)(w(r)) & / w(r) \\
& =r^{-2}\left(\Delta_{g_{+}}(\log w)-|d \log w|_{g_{+}}^{2}\right)
\end{aligned}
$$

Proof. On the one hand, the formulas

$$
\Delta_{g_{r}}=\frac{1}{\sqrt{\operatorname{det} g_{r}}} \sum_{j, k} \frac{\partial}{\partial x_{j}}\left(\sqrt{\operatorname{det} g_{r}}\left(g_{r}^{-1}\right)_{j k} \frac{\partial}{\partial x_{k}}\right)
$$

and

$$
-\delta_{g}\left(g_{r}^{-1} d\right)=\frac{1}{\sqrt{\operatorname{det} g}} \sum_{j, k} \frac{\partial}{\partial x_{j}}\left(\sqrt{\operatorname{det} g}\left(g_{r}^{-1}\right)_{j k} \frac{\partial}{\partial x_{k}}\right)
$$

imply that

$$
\Delta_{g_{r}}=-\delta_{g}\left(g_{r}^{-1} d\right)+g_{r}(d \log v, d) .
$$

Here $v$ is regarded as a function on $M$. On the other hand, a straightforward calculation shows that the Laplacian of the metric $g_{+}=r^{-2}\left(d r^{2}+g_{r}\right)$ is given by

$$
\Delta_{g_{+}}=r^{2} \frac{\partial^{2}}{\partial r^{2}}-(n-1) r \frac{\partial}{\partial r}+r^{2} \frac{\partial}{\partial r} \log v(r) \frac{\partial}{\partial r}+r^{2} \Delta_{g_{r}}
$$

Hence

$$
\frac{\partial^{2} w}{\partial r^{2}}-\frac{n-1}{r} \frac{\partial w}{\partial r}-\delta_{g}\left(g_{r}^{-1} d w\right)=\frac{1}{r^{2}}\left(\Delta_{g_{+}}(w)-g_{+}(d \log v, d w)\right),
$$

where $v$ and $w$ are regarded as functions on $X$. Therefore, we find that the left-hand side of (8.2) equals

$$
\frac{1}{r^{2}}\left(\frac{\Delta_{g_{+}}(w)}{w}-2\left|\frac{d w}{w}\right|_{g_{+}}^{2}\right) .
$$

Now a simple calculation shows that

$$
\Delta_{g_{+}}(\log w)=\frac{\Delta_{g_{+}}(w)}{w}-|d \log w|_{g_{+}}^{2} .
$$

This proves the assertion.

Theorem 8.1 contains the following two claims. 
(1) The principal part of $\mathcal{H}(r)$ is given by the self-adjoint operator

$$
-\delta\left(g_{r}^{-1} d\right) \text {. }
$$

This assertion was established in Theorem 7.1.

(2) The identity

$$
\left(\partial^{2} / \partial r^{2}-\frac{n-1}{r} \partial / \partial r+\mathcal{H}(r)\right)(w(r))=0 .
$$

Conversely, by the formal self-adjointness of all $\mathcal{M}_{2 N}$, these two properties suffice to prove Theorem 8.1. In fact, the property (1) shows that the operator $\mathcal{H}(r)+\delta\left(g_{r}^{-1} d\right)$ is self-adjoint (here $r$ is regarded as a parameter) of first-order. Hence this operator is only scalar, i.e.,

$$
\mathcal{H}(r)=-\delta\left(g_{r}^{-1} d\right)+\kappa
$$

for some $\kappa \in C^{\infty}(M)$. But $\kappa$ is determined by the action of $\mathcal{H}(r)$ on the function $w(r)$ using (8.5). This yields the assertion (8.1). Thus, using Theorem 17.1, it only remains to prove the relation (8.5).

Before we present the proof of (8.5), we discuss two important special cases of Theorem 8.1

Example 8.1. Let $M=\mathbb{S}^{n}$. Then

$$
w(r)=\left(1-r^{2} / 4\right)^{\frac{n}{2}} .
$$

In particular, $w(r)$ is constant on $\mathbb{S}^{n}$. A calculation shows that

$$
\left(\frac{\partial^{2}}{\partial r^{2}}-(n-1) r^{-1} \frac{\partial}{\partial r}\right)(w(r))=\frac{n}{2}\left(\frac{n}{2}-1\right)\left(1-r^{2} / 4\right)^{\frac{n}{2}-2} .
$$

Thus,

$$
\mathcal{H}(r)(1)=-\frac{n}{2}\left(\frac{n}{2}-1\right)\left(1-r^{2} / 4\right)^{-2}
$$

The latter formula implies

$$
\mu_{2 N}=-\frac{n}{2}\left(\frac{n}{2}-1\right) N !(N-1) ! .
$$

This fits with the summation formula

$$
\mathcal{M}_{2 N}=N !(N-1) ! P_{2}
$$

(proved in [J09b], Theorem 6.1).

The following example leaves the framework of Riemannian metrics. It shows, in a special case, that the literal extension of Theorem 8.1 remains valid for metrics with general signature (see the comments in Subsection 11.1).

Example 8.2. More generally, let $M=\mathbb{S}^{q, p}$ be the conformally flat pseudo-spheres

$$
\mathbb{S}^{(q, p)}=\mathbb{S}^{q} \times \mathbb{S}^{p}, p \geq 1, q \geq 1
$$

with the metrics $g_{\mathbb{S} q}-g_{\mathbb{S} p}$ given by the round metrics on the factors. Then

$$
w(r)=\left(1-r^{2} / 4\right)^{q / 2}\left(1+r^{2} / 4\right)^{p / 2} .
$$

In particular, $w(r)$ is constant on $\mathbb{S}^{q, p}$. Now a calculation shows that 


$$
\begin{aligned}
&\left(\frac{\partial^{2}}{\partial r^{2}}-(q+p-1) \frac{1}{r} \frac{\partial}{\partial r}\right)(w(r)) / w(r) \\
&=q / 2(q / 2-1)\left(1-r^{2} / 4\right)^{-2}-p / 2(p / 2-1)\left(1+r^{2} / 4\right)^{-2}
\end{aligned}
$$

The resulting formula for $\mathcal{H}(r)(1)$ is equivalent to

$$
-\operatorname{tr}\left(\left(\begin{array}{cc}
q / 2-1 & 0 \\
0 & p / 2-1
\end{array}\right) \mathrm{P}\left(1-r^{2} / 2 \mathrm{P}\right)^{-2}\right)
$$

(see Corollary 7.2 in $\mathrm{J09b}$ ). The latter result is confirmed by the summation formula for $\mathcal{M}_{2 N}$ established in [JK09].

In the following subsection, we prove (8.5) for general metrics subject to the usual conditions for the parameters $n$ and $N$. The case of general $N \geq 1$ for locally conformally flat metrics in even dimensions will be discussed in Subsection 8.2.

8.1. General metrics. The following proof of Theorem 8.1, i.e., of the relation (8.5), rests on the fact that the zeroth-order terms $\mu_{2 N}=\mathcal{M}_{2 N}(1)$ are determined by recursive formulas in terms of compositions of lower-order operators $\mathcal{M}_{2 M}$ acting on lower-order $\mu_{2 M}$. In the critical case $2 N=n$, such a recursive formulas follows from the identity

$$
P_{n}=\sum_{|I|=\frac{n}{2}} n_{I} \mathcal{M}_{2 I}
$$

In fact, $P_{n}(1)=0$ implies the recursive formula

$$
0=\sum_{|I|=\frac{n}{2}} n_{I} \mathcal{M}_{2 I}(1)=\mu_{n}+\sum_{|I|+a=\frac{n}{2}, a \neq \frac{n}{2}} n_{(I, a)} \mathcal{M}_{2 I}\left(\mu_{2 a}\right)
$$

for the curvature quantity $\mu_{n}$. These recursive formulas admit generalizations to general dimensions. The first three low-order examples will also be useful for later explicit calculations.

Example 8.3. For $n \geq 2$,

$$
\mu_{2}=4\left(\frac{n}{2}-1\right) w_{2}
$$

Example 8.4. For $n \geq 3$,

$$
\left(\frac{n}{2}-1\right) \mu_{4}+\mathcal{M}_{2}\left(\mu_{2}\right)=2 ! 2^{4}\left(\frac{n}{2}-1\right)\left(\frac{n}{2}-2\right) w_{4} .
$$

Proof. The definition $\mathcal{M}_{4}=P_{4}-P_{2}^{2}$ gives

$$
\mu_{4}=\left(\frac{n}{2}-2\right) Q_{4}+\left(\frac{n}{2}-1\right) P_{2}\left(Q_{2}\right) .
$$

Hence the universal recursive formula

$$
Q_{4}=-P_{2}\left(Q_{2}\right)+32 w_{4}
$$

(see Example 9.1) implies

$$
\begin{aligned}
\mu_{4} & =-\left(\frac{n}{2}-2\right) P_{2}\left(Q_{2}\right)+32\left(\frac{n}{2}-2\right) w_{4}+\left(\frac{n}{2}-1\right) P_{2}\left(Q_{2}\right) \\
& =P_{2}\left(Q_{2}\right)+32\left(\frac{n}{2}-2\right) w_{4} .
\end{aligned}
$$


It follows that

$$
\left(\frac{n}{2}-1\right) \mu_{4}+\mathcal{M}_{2}\left(\mu_{2}\right)=32\left(\frac{n}{2}-1\right)\left(\frac{n}{2}-2\right) w_{4}
$$

using

$$
\mu_{2}=-\left(\frac{n}{2}-1\right) Q_{2}
$$

The proof is complete.

But Example 8.3 shows that Eq. (8.7) is equivalent to

$$
\mathcal{M}_{4}(1)+4 \mathcal{M}_{2}\left(w_{2}\right)=32\left(\frac{n}{2}-2\right) w_{4} .
$$

Example 8.5. For even $n \geq 6$ and odd $n \geq 3$,

$$
\begin{aligned}
\frac{1}{2}\left(\frac{n}{2}-1\right)\left(\frac{n}{2}-2\right) \mu_{6}+ & \left(\frac{n}{2}-1\right) \mathcal{M}_{2}\left(\mu_{4}\right) \\
& +2\left(\frac{n}{2}-2\right) \mathcal{M}_{4}\left(\mu_{2}\right)+\mathcal{M}_{2}^{2}\left(\mu_{2}\right) \\
& =3 ! 2^{6}\left(\frac{n}{2}-1\right)\left(\frac{n}{2}-2\right)\left(\frac{n}{2}-3\right) w_{6} .
\end{aligned}
$$

Proof. First, we use the universal recursive formula

$$
Q_{6}=-2 P_{2}\left(Q_{4}\right)+2 P_{4}\left(Q_{2}\right)-3 P_{2}^{2}\left(Q_{2}\right)-3 ! 2 ! 2^{6} w_{6}
$$

to prove that

$$
\mu_{6}=-2 P_{2}\left(Q_{4}\right)+4 P_{4}\left(Q_{2}\right)-6 P_{2}^{2}\left(Q_{2}\right)+\left(\frac{n}{2}-3\right) 3 ! 2 ! 2^{6} w_{6} .
$$

In fact, the definition $\mathcal{M}_{6}=P_{6}-2 P_{2} P_{4}-2 P_{4} P_{2}+3 P_{2}^{3}$ gives

$$
\mu_{6}=-\left(\frac{n}{2}-3\right) Q_{6}-2\left(\frac{n}{2}-2\right) P_{2}\left(Q_{4}\right)+2\left(\frac{n}{2}-1\right) P_{4}\left(Q_{2}\right)-3\left(\frac{n}{2}-1\right) P_{2}^{2}\left(Q_{2}\right) .
$$

Combining this relation with (8.10) yields (8.11). Now applying the definition $\mathcal{M}_{4}=$ $P_{4}-P_{2}^{2}$ for $u=1$ shows that

$$
\mu_{4}=\left(\frac{n}{2}-2\right) Q_{4}+\left(\frac{n}{2}-1\right) P_{2}\left(Q_{2}\right) .
$$

It follows that the left-hand side of (8.9) equals the sum of

$$
3 ! 2^{6}\left(\frac{n}{2}-1\right)\left(\frac{n}{2}-2\right)\left(\frac{n}{2}-3\right) w_{6}
$$

and

$$
\begin{aligned}
\frac{1}{2}\left(\frac{n}{2}-1\right)\left(\frac{n}{2}-2\right)( & \left.-2 P_{2}\left(Q_{4}\right)+4 P_{4}\left(Q_{2}\right)-6 P_{2}^{2}\left(Q_{2}\right)\right) \\
& -2\left(\frac{n}{2}-2\right)\left(\frac{n}{2}-1\right)\left(P_{4}-P_{2}^{2}\right)\left(Q_{2}\right) \\
+ & \left(\frac{n}{2}-1\right) P_{2}\left(\left(\frac{n}{2}-2\right) Q_{4}+\left(\frac{n}{2}-1\right) P_{2}\left(Q_{2}\right)\right)-\left(\frac{n}{2}-1\right) P_{2}^{2}\left(Q_{2}\right) .
\end{aligned}
$$

It is easy to verify that the latter sum vanishes. The proof is complete. 
By Examples 8.3 8.4, the identity (8.11) is equivalent to

$$
\mathcal{M}_{6}(1)+4^{2} \mathcal{M}_{4}\left(w_{2}\right)+4^{2} 2^{2} \mathcal{M}_{2}\left(w_{4}\right)=3 ! 2 ! 2^{6}\left(\frac{n}{2}-3\right) w_{6}
$$

The proof of the formula in Example 8.5 combines a special case of the universal recursive formula for $Q$-curvature (1.14) with only algebraic calculations, i.e., no further properties of GJMS-operators and $Q$-curvatures were used. Next, we shall apply a similar method to prove the following general result.

Theorem 8.2. For $1 \leq N \leq \frac{n}{2}$ (if $n$ is even) and all $N \geq 1$ (if $n$ is odd),

$$
\begin{aligned}
\sum_{|I|=N} n_{I} \frac{\left(N-\left(I_{2}+\cdots+I_{r}\right)\right) \cdots\left(N-I_{r}\right)}{\left(\frac{n}{2}-\left(I_{2}+\cdots+I_{r}\right)\right) \cdots\left(\frac{n}{2}-I_{r}\right)} \mathcal{M}_{2 I}(1) & \\
& =\left(\frac{n}{2}-N\right)(N-1) ! N ! 2^{2 N} w_{2 N} .
\end{aligned}
$$

For even $n$ and $N=\frac{n}{2}$, the relation (8.13) is to be understood as

$$
\sum_{|I|=N} n_{I} \mathcal{M}_{2 I}(1)=0
$$

This identity is an immediate consequence of the inversion formula (Theorem 2.1) and $P_{n}(1)=0$.

Proof. We write any quantity $\mathcal{M}_{2 I}(1)$ on the left-hand side as a linear combination of GJMS-operators acting on $Q$-curvatures by using the definition (2.6). In particular, we have the contribution

$$
\mathcal{M}_{2 N}(1)=\sum_{|I|=N} m_{I} P_{2 I}(1)
$$

Among other terms, this sum contains the term

$$
P_{2 N}(1)=(-1)^{N}\left(\frac{n}{2}-N\right) Q_{2 N}
$$

We apply the universal recursive formula (1.14) for $Q$-curvature to this term and simplify the resulting formula for $\mathcal{M}_{2 N}(1)$. We find

$$
\mathcal{M}_{2 N}(1)=D_{2 N}+\left(\frac{n}{2}-N\right) N !(N-1) ! 2^{2 N} w_{2 N},
$$

where

$$
D_{2 N} \stackrel{\text { def }}{=} \sum_{|I|+a=N} m_{(I, a)}(-1)^{a}(N-a) P_{2 I}\left(Q_{2 a}\right) .
$$

The relation (8.14) generalizes the obvious relation

$$
\mathcal{M}_{n}(1)=\sum_{|I|+a=\frac{n}{2}} m_{(I, a)}(-1)^{a}\left(\frac{n}{2}-a\right) P_{2 I}\left(Q_{2 a}\right) .
$$

The formula (8.14) yields the right-hand side of (8.13) together with additional terms given by GJMS-operators acting on $Q$-curvatures. It remains to prove that, in the 
sum on the left-hand side of (8.13), all these terms vanish. For this purpose, we define the polynomial

$$
S_{2 N}(T) \stackrel{\text { def }}{=} \prod_{k=1}^{N-1}(T-k) \sum_{|I|=N} n_{I} \frac{\left(N-\left(I_{2}+\cdots+I_{r}\right)\right) \cdots\left(N-I_{r}\right)}{\left(T-\left(I_{2}+\cdots+I_{r}\right)\right) \cdots\left(T-I_{r}\right)} \mathcal{M}_{2 I}(1)
$$

of degree $N-1$. Then we have

$$
S_{2 N}\left(\frac{n}{2}\right)=\prod_{k=1}^{N-1}\left(\frac{n}{2}-k\right) \times \text { left-hand side of (18.13) }
$$

We also let $\tilde{S}_{2 N}(T)$ be the polynomial of degree $N$ which arises from $S_{2 N}(T)$ by replacing in each term the most right factor $P_{2 M}(1)$ by $(-1)^{M}(T-M) Q_{2 M}$. Then $\tilde{S}_{2 N}\left(\frac{n}{2}\right)=S_{2 N}\left(\frac{n}{2}\right)$. In these terms, Eq. (8.14) implies that

$$
\begin{aligned}
S_{2 N}\left(\frac{n}{2}\right)=N !(N-1) ! 2^{2 N} \prod_{k=1}^{N}\left(\frac{n}{2}-k\right) & w_{2 N} \\
& +\prod_{k=1}^{N-1}\left(\frac{n}{2}-k\right)\left(D_{2 N}-\mathcal{M}_{2 N}(1)\right)+\tilde{S}_{2 N}\left(\frac{n}{2}\right),
\end{aligned}
$$

i.e.,

$$
\begin{aligned}
S_{2 N}\left(\frac{n}{2}\right)=N !(N-1) ! 2^{2 N} & \prod_{k=1}^{N}\left(\frac{n}{2}-k\right) w_{2 N} \\
& +\left.\left(\prod_{k=1}^{N-1}(T-k)\left(D_{2 N}-\mathcal{M}_{2 N}(T, 1)\right)+\tilde{S}_{2 N}(T)\right)\right|_{T=\frac{n}{2}}
\end{aligned}
$$

where

$$
\mathcal{M}_{2 N}(T, 1) \stackrel{\text { def }}{=} \sum_{|I|+a=N} m_{(I, a)}(-1)^{a}(T-a) P_{2 I}\left(Q_{2 a}\right) .
$$

Now we consider the leading coefficient of the degree $N$ polynomial

$$
T \mapsto \prod_{k=1}^{N-1}(T-k)\left(D_{2 N}-\mathcal{M}_{2 N}(T, 1)\right)+\tilde{S}_{2 N}(T) .
$$

By (8.15) and (8.17), this coefficient equals

$$
-\sum_{|I|+a=N} m_{(I, a)}(-1)^{a} P_{2 I}\left(Q_{2 a}\right)+\sum_{|I|+a=N} m_{(I, a)}(-1)^{a} P_{2 I}\left(Q_{2 a}\right)=0 ;
$$

note that the sum $\tilde{S}_{2 N}(T)$ contributes to this coefficient only through the trivial composition $I=(N)$, i.e., through $\mathcal{M}_{2 N}(1)$. Thus, the polynomial (8.18) has degree $N-1$. By definition of $D_{2 N}$, we have

$$
D_{2 N}-\mathcal{M}_{2 N}(N, 1)=0 .
$$

Combining this property with

$$
\tilde{S}_{2 N}(1)=\cdots=\tilde{S}_{2 N}(N)=0,
$$


we conclude that the polynomial (8.18) has zeros at $T=1, \ldots, N$. Thus, it vanishes identically. In particular, it vanishes at $T=\frac{n}{2}$. Hence (8.16) simplifies to

$$
S_{2 N}\left(\frac{n}{2}\right)=N !(N-1) ! 2^{2 N} \prod_{k=1}^{N}\left(\frac{n}{2}-k\right) w_{2 N}
$$

Therefore, in order to complete the proof, it only remains to prove (8.20). We shall establish (8.20) by induction on $N$. The main observation is that the polynomials $S_{2 N}(T)$ satisfy the factorization relations

$$
S_{2 N}(N-a)=\left(\sum_{|J|=a} b_{J} n_{(J, N-a)} \mathcal{M}_{2 J}\right) S_{2 N-2 a}(N-a)
$$

for $a=1, \ldots, N-1$, where

$$
b_{J} \stackrel{\text { def }}{=}(-1)^{r-a} a !\left(\frac{J_{1}\left(J_{1}+J_{2}\right) \cdots\left(J_{1}+\cdots+J_{r-1}\right)}{\left(J_{2}+\cdots+J_{r}\right)\left(J_{3}+\cdots+J_{r}\right) \cdots J_{r}}\right)
$$

for $J=\left(J_{1}, \ldots, J_{r}\right)$ with $|J|=a$.

The simplest of these relations states that

$$
S_{2 N}(N-1)=(N-1) \mathcal{M}_{2} S_{2 N-2}(N-1) .
$$

In order to prove (8.21), it suffices to prove that, for all compositions $J$ and $K$ with $|J|=a$ and $|K|=N-a$, the coefficients of $\mathcal{M}_{2 J} \mathcal{M}_{2 K}(1)$ on both sides of (8.21) coincide. On the left-hand side, the coefficient for $J=\left(J_{1}, \ldots, J_{r}\right)$ and $K=$ $\left(K_{1}, \ldots, K_{s}\right)$ is given by the product of

$$
\left.\left(\frac{\left(N-\left(J_{2}+\cdots+K_{s}\right)\right) \cdots\left(N-\left(K_{1}+\cdots+K_{s}\right)\right) \cdots\left(N-K_{s}\right)}{\left(T-\left(J_{2}+\cdots+K_{s}\right)\right) \cdots\left(T-\left(K_{1}+\cdots+K_{s}\right)\right) \cdots\left(T-K_{s}\right)} \prod_{k=1}^{N-1}(T-k)\right)\right|_{T=N-a}
$$

and $n_{(J, K)}$. By $|J|=a$ and $|K|=N-a$, this product equals

$$
\begin{aligned}
J_{1} \cdots\left(J_{1}+\cdots+\right. & \left.J_{r-1}\right) a\left(a+K_{1}\right) \cdots\left(a+K_{1}+\cdots+K_{s-1}\right) \\
& \times(-1)^{r-a} \frac{(N-a-1) !(a-1) !}{\left(J_{2}+\cdots+J_{r}\right) \cdots J_{r} K_{1} \cdots\left(K_{1}+\cdots+K_{s-1}\right)} n_{(J, K)} .
\end{aligned}
$$

On the other hand, on the right-hand side of (8.21), the quantity $\mathcal{M}_{2 J} \mathcal{M}_{2 K}(1)$ contributes with the coefficient

$$
(-1)^{r-a} a !(N-1-a) !\left(\frac{J_{1} \cdots\left(J_{1}+\cdots+J_{r-1}\right)}{\left(J_{2}+\cdots+J_{r}\right) \cdots J_{r}}\right) n_{(J, N-a)} n_{K} .
$$

It follows that the equality of both expressions is equivalent to the identity

$$
\left(\frac{\left(a+K_{1}\right) \cdots\left(a+K_{1}+\cdots+K_{s-1}\right)}{K_{1} \cdots\left(K_{1}+\cdots+K_{s-1}\right)}\right) n_{(J, K)}=n_{(J, N-a)} n_{K} .
$$

But the latter relation actually follows by a straightforward calculation using (2.3). We omit the details. This proves (8.21). An analogous proof yields the factorization 
relations

$$
\tilde{S}_{2 N}(N-a)=\left(\sum_{|J|=a} b_{J} n_{(J, N-a)} \mathcal{M}_{2 J}\right) \tilde{S}_{2 N-2 a}(N-a)
$$

for $a=1, \ldots, N-1$. Now assume that we have proved (8.20) for the polynomials $\tilde{S}_{2}, \ldots, \tilde{S}_{2 N-2}$. Thus, in particular, we assume that

$$
\tilde{S}_{2}(1)=\cdots=\tilde{S}_{2 N-2}(N-1)=0 .
$$

Then the identities (8.23) (for $a=1, \ldots, N-1$ ) imply that

$$
\tilde{S}_{2 N}(1)=\cdots=\tilde{S}_{2 N}(N-1)=0 .
$$

Finally, the inversion theorem (and its proof) show that in the sum $\tilde{S}_{2 N}(N)$ the only non-trivial contribution can come from the operator $P_{2 N}$. But this contribution equals

$$
\left.(N-1) !(-1)^{N}(T-N) Q_{2 N}\right|_{T=N}=0 .
$$

Hence $\tilde{S}_{2 N}(N)=0$. This proves (18.20) for $\tilde{S}_{2 N}$. This completes the induction and the proof.

Remark 8.1. The proof of Theorem 8.2 utilizes the vanishing results (8.20). We illustrate these results by a direct verification of the relations

$$
\tilde{S}_{6}(1)=\tilde{S}_{6}(2)=\tilde{S}_{6}(3)=0 .
$$

By (8.15), the quadratic polynomial $S_{6}(T)$ is given by

$$
\begin{aligned}
& (T-1)(T-2) \\
& \quad \times\left(\mathcal{M}_{6}(1)+\frac{4}{T-1} \mathcal{M}_{4} \mathcal{M}_{2}(1)+\frac{2}{T-2} \mathcal{M}_{2} \mathcal{M}_{4}(1)+\frac{2}{(T-1)(T-2)} \mathcal{M}_{2}^{3}(1)\right) .
\end{aligned}
$$

Now the definitions of the operators $\mathcal{M}_{2}, \mathcal{M}_{4}$ and $\mathcal{M}_{6}$ yield

$$
\begin{aligned}
S_{6}(T)=(T-1)(T-2)\left[P_{6}(1)-2 P_{4} P_{2}(1)-2 P_{2} P_{4}(1)+3 P_{2}^{3}(1)\right. \\
\left.\quad+\frac{4}{T-1}\left(P_{4}-P_{2}^{2}\right) P_{2}(1)+\frac{2}{T-2} P_{2}\left(P_{4}-P_{2}^{2}\right)(1)+\frac{2}{(T-1)(T-2)} P_{2}^{3}(1)\right] .
\end{aligned}
$$

Thus, for the cubic polynomial $\tilde{S}_{6}(T)$ we obtain

$$
\begin{aligned}
\tilde{S}_{6}(T)= & (T-1)(T-2)\left[-(T-3) Q_{6}+2(T-1) P_{4}\left(Q_{2}\right)-2(T-2) P_{2}\left(Q_{4}\right)-3(T-1) P_{2}^{2}\left(Q_{2}\right)\right. \\
& \left.-4\left(P_{4}-P_{2}^{2}\right)\left(Q_{2}\right)+\frac{2}{T-2} P_{2}\left((T-2) Q_{4}+(T-1) P_{2}\left(Q_{2}\right)\right)-\frac{2}{T-2} P_{2}^{2}\left(Q_{2}\right)\right] .
\end{aligned}
$$

The claim (8.24) is a direct consequence of this formula.

Now Theorem 8.2 yields the following important formula for the quantities $\mu_{2 N}=$ $\mathcal{M}_{2 N}(1)$.

Theorem 8.3. For $1 \leq N \leq \frac{n}{2}$ (if $n$ is even) and all $N \geq 1$ (if $n$ is odd),

$$
\sum_{k=0}^{N-1}\left(2^{k} \frac{(N-1) !}{(N-1-k) !}\right)^{2} \mathcal{M}_{2 N-2 k}\left(w_{2 k}\right)=\left(\frac{n}{2}-N\right)(N-1) ! N ! 2^{2 N} w_{2 N} .
$$


Proof. The left-hand side of (8.25) equals

$$
\sum_{a=1}^{N}\left(2^{N-a} \frac{(N-1) !}{(a-1) !}\right)^{2} \mathcal{M}_{2 a}\left(w_{2 N-2 a}\right) .
$$

Now we use Theorem 8.2 (for $w_{2}, \ldots, w_{2 N-2}$ ) to rewrite this sum as

$$
\begin{aligned}
\mathcal{M}_{2 N}(1) & +\sum_{a=1}^{N-1}\left(2^{N-a} \frac{(N-1) !}{(a-1) !}\right)^{2} \frac{1}{\left(\frac{n}{2}-(N-a)\right)(N-a-1) !(N-a) ! 2^{2 N-2 a}} \\
& \times \sum_{|J|=N-a} n_{J} \frac{\left(N-a-\left(J_{2}+\cdots+J_{r-1}\right)\right) \cdots\left(N-a-J_{r-1}\right)}{\left(\frac{n}{2}-\left(J_{2}+\cdots+J_{r-1}\right)\right) \cdots\left(\frac{n}{2}-J_{r-1}\right)} \mathcal{M}_{2 a} \mathcal{M}_{2 J}(1) .
\end{aligned}
$$

On the other hand, Theorem $8.2\left(\right.$ for $\left.w_{2 N}\right)$ implies that the right-hand side of (8.25) equals

$$
\begin{aligned}
& \sum_{|I|=N} n_{I} \frac{\left(N-\left(I_{2}+\cdots+I_{r}\right)\right) \cdots\left(N-I_{r}\right)}{\left(\frac{n}{2}-\left(I_{2}+\cdots+I_{r}\right)\right) \cdots\left(\frac{n}{2}-I_{r}\right)} \mathcal{M}_{2 I}(1) \\
& \quad=\mathcal{M}_{2 N}(1)+\sum_{a=1}^{N-1} \sum_{|J|=N-a} n_{(a, J)} \frac{\left(N-\left(J_{1}+\cdots+J_{r-1}\right)\right) \cdots\left(N-J_{r-1}\right)}{\left(\frac{n}{2}-\left(J_{1}+\cdots+J_{r-1}\right)\right) \cdots\left(\frac{n}{2}-J_{r-1}\right)} \mathcal{M}_{2 a} \mathcal{M}_{2 J}(1) .
\end{aligned}
$$

Thus, the assertion follows from the relation

$$
\begin{aligned}
&\left(\frac{(N-1) !}{(a-1) !}\right)^{2} \frac{1}{\left(\frac{n}{2}-(N-a)\right)(N-a-1) !(N-a) !} \\
& \times \frac{\left(N-a-\left(J_{2}+\cdots+J_{r-1}\right)\right) \cdots\left(N-a-J_{r-1}\right)}{\left(\frac{n}{2}-\left(J_{2}+\cdots+J_{r-1}\right)\right) \cdots\left(\frac{n}{2}-J_{r-1}\right)} n_{J} \\
& \quad=\frac{\left(N-\left(J_{1}+\cdots+J_{r-1}\right)\right) \cdots\left(N-J_{r-1}\right)}{\left(\frac{n}{2}-\left(J_{1}+\cdots+J_{r-1}\right)\right) \cdots\left(\frac{n}{2}-J_{r-1}\right)} n_{(a, J)}
\end{aligned}
$$

for all $a=1, \ldots, N-1$ and all compositions $J$ of size $N-a$. For the proof of (8.27), we make the identity fully explicit by using (2.3) . Indeed, for $J=\left(J_{1}, \ldots, J_{r-1}\right)$ with $|J|=N-a$, Eq. (8.27) is equivalent to

$$
\begin{aligned}
& \left(\frac{(N-a-1) !(N-1) !}{(a-1) !}\right)^{2} \prod_{k=1}^{r-1} \frac{1}{\left(J_{k}-1\right) !^{2}} \\
& \quad \times\left(\frac{1}{J_{1}\left(J_{1}+J_{2}\right) \cdots\left(J_{1}+\cdots+J_{r-2}\right)}\right)\left(\frac{1}{\left(J_{2}+\cdots+J_{r-1}\right) \cdots J_{r-1}}\right) \\
& \quad \times \frac{\left(N-a-\left(J_{2}+\cdots+J_{r-1}\right)\right) \cdots\left(N-a-J_{r-1}\right)}{\left(\frac{n}{2}-\left(J_{2}+\cdots+J_{r-1}\right)\right) \cdots\left(\frac{n}{2}-J_{r-1}\right)} \\
& \quad=(N-1) !^{2} \frac{1}{(a-1) !^{2}} \prod_{k=1}^{r-1} \frac{1}{\left(J_{k}-1\right) !^{2}} \\
& \times \frac{1}{a\left(a+J_{1}\right) \cdots\left(a+\left(J_{1}+\cdots+J_{r-2}\right)\right)} \frac{1}{\left(J_{1}+\cdots+J_{r-1}\right)\left(J_{2}+\cdots+J_{r-1}\right) \cdots J_{r-1}}
\end{aligned}
$$




$$
\times \frac{\left(N-\left(J_{1}+\cdots+J_{r-1}\right)\right) \cdots\left(N-J_{r-1}\right)}{\left(\frac{n}{2}-\left(J_{1}+\cdots+J_{r-1}\right)\right)\left(\frac{n}{2}-\left(J_{2}+\cdots+J_{r-1}\right)\right) \cdots\left(\frac{n}{2}-J_{r-1}\right)} .
$$

Now using $\frac{n}{2}-\left(J_{1}+\cdots+J_{r-1}\right)=\frac{n}{2}-(N-a)$,

$$
\frac{\left(N-a-\left(J_{2}+\cdots+J_{r-1}\right)\right) \cdots\left(N-a-J_{r-1}\right)}{J_{1}\left(J_{1}+J_{2}\right) \cdots\left(J_{1}+\cdots+J_{r-2}\right)}=1
$$

and

$$
\frac{\left(N-\left(J_{1}+\cdots+J_{r-1}\right)\right) \cdots\left(N-J_{r-1}\right)}{a\left(a+J_{1}\right) \cdots\left(a+\left(J_{1}+\cdots+J_{r-2}\right)\right)}=1,
$$

it is easy to verify (8.28). The proof is complete.

Now we are ready to prove Theorem 8.1

Proof of Theorem 8.1. By definition of $\mathcal{H}(r)$, we have

$$
\begin{aligned}
\mathcal{H}(r)(w(r)) & =\left(\sum_{N \geq 0} \mathcal{M}_{2 N+2} \frac{1}{N !^{2}}\left(\frac{r^{2}}{4}\right)^{N}\right)\left(\sum_{M \geq 0} w_{2 M} r^{2 M}\right) \\
& =\sum_{N, M \geq 0} 2^{2 M} \frac{1}{N !^{2}} \mathcal{M}_{2 N+2}\left(w_{2 M}\right)\left(\frac{r^{2}}{4}\right)^{N+M} \\
& =\sum_{T \geq 1}\left(\sum_{S=0}^{T-1} 2^{2 S} \frac{1}{(T-1-S) !^{2}} \mathcal{M}_{2 T-2 S}\left(w_{2 S}\right)\right)\left(\frac{r^{2}}{4}\right)^{T-1} \\
& =\sum_{T \geq 1} 2 T(n-2 T) w_{2 T} r^{2 T-2} .
\end{aligned}
$$

In the last step we have used Theorem 8.3. But the latter sum equals

$$
\left(-\frac{\partial^{2}}{\partial r^{2}}+(n-1) \frac{1}{r} \frac{\partial}{\partial r}\right)(w(r)) \text {. }
$$

This proves (8.5).

Remark 8.2. For the round sphere $\mathbb{S}^{n}$, the summation formula (Theorem 6.1 in [J09b])

$$
\mathcal{M}_{2 N}=N !(N-1) ! P_{2}
$$

and

$$
w(r)=\left(1-\frac{r^{2}}{4}\right)^{\frac{n}{2}}
$$

show that Theorem 8.3 is equivalent to

$$
\begin{aligned}
-\sum_{k=0}^{N-1}\left(2^{k}\left(\begin{array}{c}
N-1 \\
k
\end{array}\right)\right. & k !)^{2} \frac{n}{2}\left(\frac{n}{2}-1\right)(N-k) !(N-k-1) ! \\
& \times\left((-1)^{k}\left(\begin{array}{c}
\frac{n}{2} \\
k
\end{array}\right) 2^{-2 k}\right)=(-1)^{N}\left(\frac{n}{2}-N\right)(N-1) ! N !\left(\begin{array}{c}
\frac{n}{2} \\
N
\end{array}\right) .
\end{aligned}
$$


The latter relation is a consequence of the well-known summation formula

$$
\sum_{k=0}^{N-1}\left(\begin{array}{l}
\frac{n}{2} \\
k
\end{array}\right)(-1)^{k}=(-1)^{N-1}\left(\begin{array}{c}
\frac{n}{2}-1 \\
N-1
\end{array}\right)
$$

(GKP], Eq. (5.16)). Similarly, for an Einstein metric $g$ with $\operatorname{Ric}(g)=\lambda g$, we have

$$
\mathrm{P}=\frac{\lambda}{2(n-1)} g=\frac{c}{2} g \quad \text { with } \quad c=\frac{\text { scal }}{n(n-1)}
$$

and

$$
w(r)=\operatorname{det}\left(1-\frac{r^{2}}{2} \mathrm{P}\right)=\left(1-\frac{r^{2}}{4} c\right)^{\frac{n}{2}} .
$$

Moreover, by a rescaling argument, we find

$$
\mathcal{M}_{2 N}=N !(N-1) ! c^{N-1} P_{2},
$$

and it follows that the identity (8.29) also confirms Theorem8.3 for Einstein metrics.

For locally conformally flat metrics, Theorem 1.1 extends to super-critical GJMSoperators in even dimensions. For the proof of this claim it would be enough to extend the relations (8.25). However, the above proof of (8.25) does not extend since the formulation of Theorem 8.2 does not make sense if $2 N>n$. Therefore, in the following subsection, we shall provide an alternative proof of (8.25) for locally conformally flat metrics which extends to $2 N>n$.

8.2. Conformally flat metrics. In the present section, we extend Theorem 8.3 for locally conformally flat metrics to all $N \geq 1$. We shall derive this fact from the restriction property and the commutator relations of the operators $\mathcal{M}_{2 N}$. We recall that these relations are not obstructed for large $N$.

We start by proving the following result.

Lemma 8.2. For $N \geq 3$, we have

$$
\mathcal{M}_{2 N} i^{*}=(2 N-2)\left(i^{*}\left[\frac{\partial^{2}}{\partial r^{2}}, \overline{\mathcal{M}}_{2 N-2}\right]-2\left[\mathcal{M}_{2 N-2}, w_{2}\right] i^{*}\right) .
$$

Proof. The restriction property (see Theorem 6.3)

$$
i^{*} \overline{\mathcal{M}}_{2 N-2}=\mathcal{M}_{2 N-2} i^{*}, N \geq 3
$$

shows that

$$
\begin{aligned}
& i^{*}\left[\bar{P}_{2}, \overline{\mathcal{M}}_{2 N-2}\right]-\left[P_{2}, \mathcal{M}_{2 N-2}\right] i^{*} \\
& =i^{*} \bar{P}_{2} \overline{\mathcal{M}}_{2 N-2}-i^{*} \overline{\mathcal{M}}_{2 N-2} \bar{P}_{2}-P_{2} \mathcal{M}_{2 N-2} i^{*}+\mathcal{M}_{2 N-2} P_{2} i^{*} \\
& =i^{*} \bar{P}_{2} \overline{\mathcal{M}}_{2 N-2}-\mathcal{M}_{2 N-2} i^{*} \bar{P}_{2}-P_{2} i^{*} \overline{\mathcal{M}}_{2 N-2}+\mathcal{M}_{2 N-2} P_{2} i^{*} \\
& =\left(i^{*} \bar{P}_{2}-P_{2} i^{*}\right) \overline{\mathcal{M}}_{2 N-2}-\mathcal{M}_{2 N-2}\left(i^{*} \bar{P}_{2}-P_{2} i^{*}\right) .
\end{aligned}
$$

Moreover, the formulas

$$
i^{*} \bar{P}_{2}=i^{*} \frac{\partial^{2}}{\partial r^{2}}+\Delta i^{*}-\frac{n-1}{2} i^{*} \bar{\jmath} \quad \text { and } \quad P_{2}=\Delta-\frac{n-2}{2} \mathrm{~J}
$$


and the relation $i^{*} \overline{\mathrm{J}}=\mathrm{J}$ imply that

$$
i^{*} \bar{P}_{2}-P_{2} i^{*}=i^{*}\left(\frac{\partial^{2}}{\partial r^{2}}-\frac{1}{2} \overline{\mathrm{J}}\right) .
$$

Another application of (8.31) shows that (8.32) implies

$$
i^{*}\left[\bar{P}_{2}, \overline{\mathcal{M}}_{2 N-2}\right]-\left[P_{2}, \mathcal{M}_{2 N-2}\right] i^{*}=i^{*}\left(\frac{\partial^{2}}{\partial r^{2}}-\frac{1}{2} \bar{\jmath}\right) \overline{\mathcal{M}}_{2 N-2}-i^{*} \overline{\mathcal{M}}_{2 N-2}\left(\frac{\partial^{2}}{\partial r^{2}}-\frac{1}{2} \bar{J}\right) .
$$

Now the assertion follows by combining this identity with the commutator relation (6.12) using (8.31) and $i^{*} \overline{\mathrm{J}}=\mathrm{J}$.

By applying (8.30) to $u=1$, we find the following recursive formula.

Lemma 8.3. We have

$$
\mu_{2 N}=(2 N-2)\left(i^{*} \frac{\partial^{2}}{\partial r^{2}}\left(\bar{\mu}_{2 N-2}\right)-2 \mathcal{M}_{2 N-2}^{0}\left(w_{2}\right)\right)
$$

for $N \geq 3$.

Example 8.6. Let $N=3$. Then Lemma 8.3 and the explicit formula (10.12) for $\mu_{4}$ show that

$$
\mu_{6}=4 i^{*} \frac{\partial^{2}}{\partial r^{2}}\left(-\overline{\mathrm{J}}^{2}-(n-3)|\overline{\mathrm{P}}|^{2}+\bar{\Delta}(\overline{\mathrm{J}})\right)-8 \delta(\mathrm{P} d \mathrm{~J}) .
$$

In fact, using formulas in Sections 12.2]12.3, one can verify that (8.34) is equivalent to $(10.13)$.

These preparations enable us to prove the main result of the present section.

Theorem 8.4. For any locally conformally flat metric $g$, we have

$$
\mu_{2 N}+\sum_{k=1}^{N-1}\left(2^{k} \frac{(N-1) !}{(N-1-k) !}\right)^{2} \mathcal{M}_{2 N-2 k}\left(w_{2 k}\right)=\left(\frac{n}{2}-N\right)(N-1) ! N ! 2^{2 N} w_{2 N}
$$

for all $N \geq 1$.

Proof. We use induction on $N$. For $N=1$ and $N=2$, the assertions can be easily verified by direct calculations. In fact, for $N=1$, the assertion is the obvious relation

$$
\mu_{2}=4\left(\frac{n}{2}-1\right) w_{2}
$$

(see Example 8.3). Similarly, for $N=2$ the assertion coincides with Example 8.4. Now assume that (8.35) has been proved up to $\mu_{2 N-2}$ (for conformally flat metrics). The metric $\bar{g}$ is locally conformally flat, too. Hence, by (8.33), the assertion for $\mu_{2 N}$ is equivalent to the relation

$$
\begin{aligned}
\left(\frac{n}{2}-N\right) & (N-1) ! N ! 2^{2 N} w_{2 N}-\sum_{k=1}^{N-1}\left(2^{k} \frac{(N-1) !}{(N-1-k) !}\right)^{2} \mathcal{M}_{2 N-2 k}\left(w_{2 k}\right) \\
& =(2 N-2)\left[\frac { \partial ^ { 2 } } { \partial r ^ { 2 } } | _ { r = 0 } \left(-\sum_{k=1}^{N-2}\left(2^{k} \frac{(N-2) !}{(N-2-k) !}\right)^{2} \overline{\mathcal{M}}_{2 N-2-2 k}\left(\bar{w}_{2 k}\right)\right.\right.
\end{aligned}
$$




$$
\begin{aligned}
& \left.+\left(\frac{n+3}{2}-N\right)(N-2) !(N-1) ! 2^{2 N-2} \bar{w}_{2 N-2}\right) \\
& -2 \mathcal{M}_{2 N-2}\left(w_{2}\right) \\
& +2\left(-\sum_{k=1}^{N-2}\left(2^{k} \frac{(N-2) !}{(N-2-k) !}\right)^{2} \mathcal{M}_{2 N-2-2 k}\left(w_{2 k}\right)\right. \\
& \left.\left.+\left(\frac{n+2}{2}-N\right)(N-2) !(N-1) ! 2^{2 N-2} w_{2 N-2}\right) w_{2}\right] .
\end{aligned}
$$

Here we use the notation $\bar{w}_{2 N}$ for the Taylor coefficients of $\bar{w}(r)=w\left(d r^{2}+g(r)\right)$. Now Lemma 8.2 and the restriction property (Theorem 6.3) imply

$$
\begin{aligned}
\left.(2 N-2 k-2) \frac{\partial^{2}}{\partial r^{2}}\right|_{r=0}\left(\overline{\mathcal{M}}_{2 N-2 k-2}\left(\bar{w}_{2 k}\right)\right) & =(2 N-2 k-2) \mathcal{M}_{2 N-2 k-2}\left(\left.\frac{\partial^{2}}{\partial r^{2}}\right|_{r=0}\left(\bar{w}_{2 k}\right)\right) \\
& +\mathcal{M}_{2 N-2 k}\left(w_{2 k}\right)-2(2 N-2 k-2)\left[w_{2}, \mathcal{M}_{2 N-2 k-2}\right]\left(w_{2 k}\right)
\end{aligned}
$$

if $2 N-2 k-2 \geq 4$. Next, we observe that

$$
\left.\frac{\partial^{2}}{\partial r^{2}}\right|_{r=0}(w(r, s))=\frac{1}{s} \frac{\partial w(s)}{\partial s}-2 w_{2} w(s)
$$

(by the first part of Lemma 8.6). By comparing coefficients of powers of $s$ in (8.38), we obtain

$$
\left.\frac{\partial^{2}}{\partial r^{2}}\right|_{r=0}\left(\bar{w}_{2 k}\right)=(2 k+2) w_{2 k+2}-2 w_{2} w_{2 k}, k \geq 0 .
$$

Hence (8.37) implies

$$
\begin{aligned}
& \left.(2 N-2 k-2) \frac{\partial^{2}}{\partial r^{2}}\right|_{r=0}\left(\overline{\mathcal{M}}_{2 N-2 k-2}\left(\bar{w}_{2 k}\right)\right)=\mathcal{M}_{2 N-2 k}\left(w_{2 k}\right) \\
& +(2 N-2 k-2)(2 k+2) \mathcal{M}_{2 N-2 k-2}\left(w_{2 k+2}\right)-2(2 N-2 k-2) \mathcal{M}_{2 N-2 k-2}\left(w_{2 k}\right) w_{2}
\end{aligned}
$$

if $2 N-2 k-2 \geq 4$. The relations (8.39) for $k=N-1$ and (8.40) for $k=1, \ldots, N-2$ show that the right-hand side of (8.36) $)$ equals the product of $(2 N-2)$ and the sum

$$
\begin{aligned}
& {\left[-\frac{2^{2}(N-2)^{2}}{(2 N-4)}\left(\mathcal{M}_{2 N-2}\left(w_{2}\right)+(2 N-4) 4 \mathcal{M}_{2 N-4}\left(w_{4}\right)-2(2 N-4) \mathcal{M}_{2 N-4}\left(w_{2}\right) w_{2}\right)\right.} \\
& \vdots \\
& -\frac{2^{2(N-3)}(N-2) !^{2}}{4}\left(\mathcal{M}_{6}\left(w_{2 N-6}\right)+4(2 N-4) \mathcal{M}_{4}\left(w_{2 N-4}\right)-8 \mathcal{M}_{4}\left(w_{2 N-6}\right) w_{2}\right) \\
& -\left.2^{2(N-2)}(N-2) !^{2} \frac{\partial^{2}}{\partial r^{2}}\right|_{r=0} \overline{\mathcal{M}}_{2}\left(\bar{w}_{2 N-4}\right) \\
& +\left(\frac{n+3}{2}-N\right)(N-2) !(N-1) ! 2^{2 N-2}\left(2 N w_{2 N}-2 w_{2 N-2} w_{2}\right) \\
& -2 \mathcal{M}_{2 N-2}\left(w_{2}\right) \\
& +2\left(-\sum_{k=1}^{N-2}\left(2^{k} \frac{(N-2) !}{(N-2-k) !}\right)^{2} \mathcal{M}_{2 N-2-2 k}\left(w_{2 k}\right)\right.
\end{aligned}
$$




$$
\left.\left.+\left(\frac{n+2}{2}-N\right)(N-2) !(N-1) ! 2^{2 N-2} w_{2 N-2}\right) w_{2}\right] .
$$

The latter formula shows that the assertion $(\underline{8.36})$ is equivalent to a formula for

$$
\left.2^{2 N-3}(N-1) !(N-2) ! \frac{\partial^{2}}{\partial r^{2}}\right|_{r=0} \overline{\mathcal{M}}_{2}\left(\bar{w}_{2 N-4}\right) .
$$

in terms of the quantities

$$
w_{2 N}, \mathcal{M}_{2}\left(w_{2 N-2}\right), \mathcal{M}_{4}\left(w_{2 N-4}\right)
$$

and

$$
w_{2 N-2} w_{2}, \quad \mathcal{M}_{2}\left(w_{2 N-4}\right) w_{2}
$$

all other contributions cancel. More precisely, the respective coefficients of these five terms are given by

$$
2^{2 N-1} 3 N !(N-1) !, \quad 2^{2 N-2}(N-1) !^{2}, \quad 2^{2 N-4}(N-1) !(N-2) !
$$

and

$$
-2^{2 N-1}(N-1) !^{2}, \quad-2^{2 N-2}(N-1) !(N-2) !
$$

This reduces the proof of (8.36) to the following identity.

Lemma 8.4. For $N \geq 3$, we have

$$
\begin{array}{r}
\left.\frac{\partial^{2}}{\partial r^{2}}\right|_{r=0}\left(\overline{\mathcal{M}}_{2}\left(\bar{w}_{2 N-4}\right)\right)=12 N(N-1) w_{2 N}+(2 N-2) \mathcal{M}_{2}\left(w_{2 N-2}\right)+\frac{1}{2} \mathcal{M}_{4}\left(w_{2 N-4}\right) \\
-2(2 N-2) w_{2 N-2} w_{2}-2 \mathcal{M}_{2}\left(w_{2 N-4}\right) w_{2} .
\end{array}
$$

Next we use explicit formulas for $\mathcal{M}_{2}$ and $\mathcal{M}_{4}$ to reduce Lemma 8.4 to the following result.

Lemma 8.5. For $N \geq 2$, we have

$$
\left.\frac{\partial^{4}}{\partial r^{4}}\right|_{r=0}\left(\bar{w}_{2 N-4}\right)=12 N(N-1) w_{2 N}+24\left(w_{2}^{2}-w_{4}\right) w_{2 N-4}-12(2 N-2) w_{2} w_{2 N-2} .
$$

In order to prove that Lemma 8.5 implies Lemma 8.4, we use the formula

$$
\overline{\mathcal{M}}_{2}=\frac{\partial^{2}}{\partial r^{2}}+\frac{\dot{v}}{v}(r) \frac{\partial}{\partial r}+\Delta_{g_{r}}-\frac{n-1}{2} \overline{\mathrm{J}}
$$

(see Eq. (7.12)). It follows that the left-hand side of (8.41) equals the sum

$$
\begin{aligned}
& \left.\frac{\partial^{4}}{\partial r^{4}}\right|_{r=0}\left(\bar{w}_{2 N-4}\right)+\left.\left.2 \frac{\partial}{\partial r}\right|_{r=0}\left(\frac{\dot{v}}{v}\right) \frac{\partial^{2}}{\partial r^{2}}\right|_{r=0}\left(\bar{w}_{2 N-4}\right) \\
& \quad-2 \delta(\mathrm{P} d)\left(w_{2 N-4}\right)-\left(d \mathrm{~J}, d w_{2 N-4}\right)+\left.\Delta_{g} \frac{\partial^{2}}{\partial r^{2}}\right|_{r=0}\left(\bar{w}_{2 N-4}\right)-\left.\frac{n-1}{2} \frac{\partial^{2}}{\partial r^{2}}\right|_{r=0}\left(\overline{\mathrm{J}} \bar{w}_{2 N-4}\right) .
\end{aligned}
$$

Here we made use of the variational formula

$$
\left.(d / d t)\right|_{0}\left(\Delta_{g-t \mathrm{P}}\right)=-\delta(\mathrm{P} d)-\frac{1}{2}(d \mathrm{~J}, d)
$$

(see Eq. (6.9.33) in [J09a ). Thus by

$$
\left.(\partial / \partial r)^{2}\right|_{r=0}(\overline{\mathbf{J}})=|\mathrm{P}|^{2}
$$


(see Lemma 6.11.1 in [J09a]) the left-hand side of (8.41) equals

$$
\begin{aligned}
\left.\frac{\partial^{4}}{\partial r^{4}}\right|_{r=0}\left(\bar{w}_{2 N-4}\right)+\left.8 w_{2} \frac{\partial^{2}}{\partial r^{2}}\right|_{r=0}\left(\bar{w}_{2 N-4}\right) & \\
-2 \delta(\mathrm{P} d)\left(w_{2 N-4}\right)+4\left(d w_{2},\right. & \left.d w_{2 N-4}\right)+\left.\Delta_{g} \frac{\partial^{2}}{\partial r^{2}}\right|_{r=0}\left(\bar{w}_{2 N-4}\right) \\
& -\frac{n-1}{2}\left(|\mathrm{P}|^{2} w_{2 N-4}+\left.\mathrm{J} \frac{\partial^{2}}{\partial r^{2}}\right|_{r=0}\left(\bar{w}_{2 N-4}\right)\right) .
\end{aligned}
$$

We simplify the latter identity using the relation

$$
\left.\frac{\partial^{2}}{\partial r^{2}}\right|_{r=0}\left(\bar{w}_{2 N-4}\right)=(2 N-2) w_{2 N-2}-2 w_{2} w_{2 N-4}
$$

(see Eq. (8.39) for $k=N-2$ ). Now a calculation of the right-hand side of (8.41) using

$$
\mathcal{M}_{2}=-\delta d+4\left(\frac{n}{2}-1\right) w_{2} \quad \text { and } \quad \mathcal{M}_{4}=-4 \delta(\mathrm{P} d)-4 \mathcal{M}_{2}\left(w_{2}\right)+32\left(\frac{n}{2}-2\right) w_{4}
$$

(see Example 9.2) and

$$
\mathrm{J}=-4 w_{2} \quad \text { and } \quad|\mathrm{P}|^{2}=-16 w_{4}+8 w_{2}^{2}
$$

proves the asserted implication. We omit the details.

Finally, we turn to the proof of Lemma 8.5 (for locally conformally flat metrics).

Note that the identity (8.42) is trivial for $N=2$. We rewrite the assertion in terms of generating functions. Then

$$
\left.\frac{\partial^{4}}{\partial r^{4}}\right|_{r=0}(w(r, s))=3\left(\frac{1}{s} \frac{\partial}{\partial s}\right)^{2}(w(s))-12 w_{2}\left(\frac{1}{s} \frac{\partial}{\partial s}\right)(w(s))+24\left(w_{2}^{2}-w_{4}\right) w(s) .
$$

Now for locally conformally flat metrics, we have

$$
\begin{aligned}
w(s) & =\operatorname{det}\left(1-s^{2} / 2 \mathrm{P}\right)^{1 / 2} \\
w(r, s) & =\operatorname{det}\left(1-\left(r^{2}+s^{2}\right) / 2 \mathrm{P}\right)^{1 / 2} / \operatorname{det}\left(1-r^{2} / 2 \mathrm{P}\right)^{1 / 2} .
\end{aligned}
$$

We set

$$
\begin{aligned}
\tilde{w}(s) & \stackrel{\text { def }}{=} \operatorname{det}(1-s / 2 \mathrm{P})^{1 / 2} \\
\tilde{w}(r, s) & \stackrel{\text { def }}{=} \operatorname{det}(1-(r+s) / 2 \mathrm{P})^{1 / 2} / \operatorname{det}(1-r / 2 \mathrm{P})^{1 / 2}
\end{aligned}
$$

Hence

$$
\left.\frac{\partial^{4}}{\partial r^{4}}\right|_{r=0}(w(r, s))=\left.12 \frac{\partial^{2}}{\partial r^{2}}\right|_{r=0}\left(\tilde{w}\left(r, s^{2}\right)\right)
$$

and

$$
\frac{1}{s} \frac{\partial}{\partial s}(w(s))=2 \tilde{w}^{\prime}\left(s^{2}\right), \quad\left(\frac{1}{s} \frac{\partial}{\partial s}\right)^{2}(w(s))=4 \tilde{w}^{\prime \prime}\left(s^{2}\right) .
$$

Here' denotes the derivative with respect to $s$. Hence the assertion (8.44) is equivalent to the second part of the following result. 
Lemma 8.6. We have

$$
\left.\frac{\partial}{\partial r}\right|_{r=0}(\tilde{w}(r, s))=\tilde{w}^{\prime}(s)-\tilde{w}_{2} \tilde{w}(s)
$$

and

where

$$
\left.\frac{\partial^{2}}{\partial r^{2}}\right|_{r=0}(\tilde{w}(r, s))=\tilde{w}^{\prime \prime}(s)-2 \tilde{w}_{2} \tilde{w}^{\prime}(s)-2\left(\tilde{w}_{4}-\tilde{w}_{2}^{2}\right) \tilde{w}(s)
$$

$$
\tilde{w}(s)=1+\tilde{w}_{2} s+\tilde{w}_{4} s^{2}+\cdots
$$

Proof. The relation

$$
\tilde{w}(r, s)=\tilde{w}(r+s) / \tilde{w}(r)
$$

implies

$$
\begin{aligned}
\left.\frac{\partial}{\partial r}\right|_{r=0}(\tilde{w}(r, s)) & =\left.\frac{\partial}{\partial r}\right|_{r=0}\left(\tilde{w}(r+s)\left(1-\tilde{w}_{2} r+\cdots\right)\right) \\
& =\tilde{w}^{\prime}(s)-\tilde{w}_{2} \tilde{w}(s)
\end{aligned}
$$

and

$$
\begin{aligned}
\left.\frac{\partial^{2}}{\partial r^{2}}\right|_{r=0}(\tilde{w}(r, s)) & =\left.\frac{\partial^{2}}{\partial r^{2}}\right|_{r=0}\left(\tilde{w}(r+s)\left(1-\tilde{w}_{2} r-\left(\tilde{w}_{4}-\tilde{w}_{2}^{2}\right) r^{2}+\cdots\right)\right) \\
& \left.=\tilde{w}^{\prime \prime}(s)-2 \tilde{w}_{2} \tilde{w}^{\prime}(s)-2\left(\tilde{w}_{4}-\tilde{w}_{2}^{2}\right) \tilde{w}(s)\right) .
\end{aligned}
$$

The proof is complete.

This completes the proof Theorem 8.4.

Now, in even dimensions $n$ and locally conformally flat metrics, Theorem 8.4 extends Theorem 8.3 to super-critical orders $2 N>n$. The same arguments as in Subsection 8.1 then extend Theorem 8.1 for such metrics to all $N \geq 1$.

Remark 8.3. For even dimensions $n$ and sub-critical orders $2 N \leq n$, the proof of Theorem 8.4 extends to general metrics. In fact, it suffices to replace the usage of the explicit formulas for $w(r)$ and $w(r, s)$ by Theorem 7.2.

Finally, for locally conformally flat metrics, we use Theorem 8.1 to derive the contributions (1.11) to the zeroth-order term of $\mathcal{M}_{2 N}$.

Theorem 8.5. For locally conformally flat $g$ and all $N \geq 1$, the contribution of

$$
-\left(\frac{\partial^{2} w}{\partial r^{2}}-(n-1) \frac{1}{r} \frac{\partial w}{\partial r}\right) / w
$$

to the zeroth-order term of $\mathcal{M}_{2 N}(1)$ equals

$$
-(N-1) !^{2} 2^{N-1}\left(\left(\frac{n}{2}-N\right) \operatorname{tr}\left(\mathrm{P}^{N}\right)+\frac{1}{2} \sum_{a=1}^{N-1} \operatorname{tr}\left(\mathrm{P}^{a}\right) \operatorname{tr}\left(\mathrm{P}^{N-a}\right)\right) .
$$

Proof. By the assumption on $g$, we have $w(r)=\sqrt{\operatorname{det}\left(1-r^{2} / 2 \mathrm{P}\right)}$. Now we calculate

$$
\begin{aligned}
\frac{1}{r} \frac{1}{w} \frac{\partial w}{\partial r} & =\frac{1}{r} \frac{\partial}{\partial r}\left(\log \sqrt{\operatorname{det}\left(1-r^{2} / 2 \mathrm{P}\right)}\right) \\
& =\frac{1}{2 r} \frac{\partial}{\partial r}\left(\log \operatorname{det}\left(1-r^{2} / 2 \mathrm{P}\right)\right)
\end{aligned}
$$




$$
\begin{aligned}
& =\frac{1}{2 r} \frac{\partial}{\partial r}\left(\operatorname{tr} \log \left(1-r^{2} / 2 \mathrm{P}\right)\right) \\
& =-\frac{1}{2} \operatorname{tr}\left(\frac{\mathrm{P}}{1-r^{2} / 2 \mathrm{P}}\right) \\
& =-\frac{1}{2} \sum_{N \geq 0} \operatorname{tr}\left(\mathrm{P}^{N+1}\right)\left(\frac{r^{2}}{2}\right)^{N}
\end{aligned}
$$

and

$$
\begin{aligned}
\frac{\partial^{2}}{\partial r^{2}}(\log w) & =\frac{1}{2} \frac{\partial^{2}}{\partial r^{2}}\left(\operatorname{tr} \log \left(1-r^{2} / 2 \mathrm{P}\right)\right) \\
& =-\frac{1}{2} \frac{\partial}{\partial r}\left(\operatorname{tr}\left(\frac{r \mathrm{P}}{1-r^{2} / 2 \mathrm{P}}\right)\right) \\
& =-\frac{1}{2} \operatorname{tr}\left(\mathrm{P} \frac{1+r^{2} / 2 \mathrm{P}}{\left(1-r^{2} / 2 \mathrm{P}\right)^{2}}\right) \\
& =-\frac{1}{2} \sum_{N \geq 1}(2 N-1) \operatorname{tr}\left(\mathrm{P}^{N}\right)\left(\frac{r^{2}}{2}\right)^{N-1} .
\end{aligned}
$$

These relations and

$$
\frac{1}{w} \frac{\partial^{2} w}{\partial r^{2}}=\left(\frac{1}{w} \frac{\partial w}{\partial r}\right)^{2}+\frac{\partial^{2}}{\partial r^{2}}(\log w)
$$

imply that (8.47) equals

$$
\begin{aligned}
-\frac{1}{4} r^{2}\left(\sum_{N \geq 0} \operatorname{tr}\left(\mathrm{P}^{N+1}\right)\left(\frac{r^{2}}{2}\right)^{N}\right)^{2} & \\
& +\frac{1}{2} \sum_{N \geq 1}(2 N-1) \operatorname{tr}\left(\mathrm{P}^{N}\right)\left(\frac{r^{2}}{2}\right)^{N-1}-\frac{n-1}{2} \sum_{N \geq 0} \operatorname{tr}\left(\mathrm{P}^{N+1}\right)\left(\frac{r^{2}}{2}\right)^{N} .
\end{aligned}
$$

These sums can be simplified to

$$
-\frac{1}{2} \sum_{N \geq 1}\left(\sum_{a=1}^{N-1} \operatorname{tr}\left(\mathrm{P}^{a}\right) \operatorname{tr}\left(\mathrm{P}^{N-a}\right)\right)\left(\frac{r^{2}}{2}\right)^{N-1}+\frac{2 N-n}{2} \sum_{N \geq 1} \operatorname{tr}\left(\mathrm{P}^{N}\right)\left(\frac{r^{2}}{2}\right)^{N-1} .
$$

Now the assertion follows by taking the coefficient of $\left(r^{2} / 4\right)^{N-1}$.

For the round sphere $\mathbb{S}^{n}$, Theorem 8.5 reproves the fact that the zeroth-order term of $\mathcal{M}_{2 N}$ is given by

(see Eq. ( $(8.6))$.

$$
-(N-1) ! N ! \frac{n}{2}\left(\frac{n}{2}-1\right)
$$

\section{The EXPlicit FORMula For $Q$-CURVATURE}

As a consequence of Theorem 2.1 and Theorem 8.3, we find an explicit formula for $Q$-curvature. The following result restates Theorem 1.2 . 
Theorem 9.1. (Explicit formula for $Q$-curvature) For even $n$ and $2 N \leq n$ and for odd $n$ and all $N \geq 1$,

$$
(-1)^{N} Q_{2 N}=\sum_{|I|+a=N} n_{(I, a)} a !(a-1) ! 2^{2 a} \mathcal{M}_{2 I}\left(w_{2 a}\right) .
$$

Proof. By combining

$$
P_{2 N}(1)=(-1)^{N}\left(\frac{n}{2}-N\right) Q_{2 N}
$$

with Theorem 2.1, we find

$$
(-1)^{N}\left(\frac{n}{2}-N\right) Q_{2 N}=\sum_{|I|=N} n_{I} \mathcal{M}_{2 I}(1)=\sum_{|I|+a=N} n_{(I, a)} \mathcal{M}_{2 I}\left(\mu_{2 a}\right) .
$$

By Theorem 8.3, the latter sum equals

$$
\begin{aligned}
\sum_{|I|+a=N} n_{(I, a)} \mathcal{M}_{2 I}\left(\left(\frac{n}{2}-a\right) a !(a-1) ! 2^{2 a} w_{2 a}\right. & \\
& \left.\quad-(2 a-2)^{2} \mathcal{M}_{2 a-2}\left(w_{2}\right)-\cdots-((2 a-2) \cdots 2) \mathcal{M}_{2}\left(w_{2 a-2}\right)\right) .
\end{aligned}
$$

This sum is a linear combination of quantities of the form

$$
\mathcal{M}_{2 J} \mathcal{M}_{2 b}\left(w_{2 a}\right), \quad|J|+a+b=N
$$

with respective coefficients

$$
n_{(J, b, a)}\left(\frac{n}{2}-a\right) a !(a-1) ! 2^{2 a}-n_{(J, a+b)}((2(a+b)-2) \cdots 2 b)^{2} .
$$

Hence for $2 N \neq n$ it suffices to prove that these differences coincide with

$$
n_{(J, b, a)}\left(\frac{n}{2}-N\right) a !(a-1) ! 2^{2 a} .
$$

In turn, this claim is equivalent to the identity

$$
n_{(J, b, a)}(N-a) a !(a-1) !=n_{(J, a+b)}\left(\frac{(a+b-1) !}{(b-1) !}\right)^{2} .
$$

But, according to (2.2), the latter identity is equivalent to

$$
\left(\begin{array}{c}
N-1 \\
a+b-1
\end{array}\right)\left(\frac{(a+b-1) !}{(b-1) !}\right)^{2}=\left(\begin{array}{c}
N-a-1 \\
b-1
\end{array}\right)\left(\begin{array}{c}
b+a-1 \\
b-1
\end{array}\right)\left(\begin{array}{c}
N-1 \\
a-1
\end{array}\right)(N-a) a !(a-1) ! .
$$

This relation can be confirmed by a straightforward calculation. The critical case follows by continuation.

Some comments are in order. Of course, the actual power of Theorem 9.1 comes from the identification of the operators $\mathcal{M}_{2 N}$ as certain operators of only second order (Theorem 1.1). In connection with further evaluations of Theorem 9.1, it is useful to recall that Graham [G09] gave an algorithm which generates formulas for the Taylor coefficients $v_{2 j}$ of $v(r)$ in terms of the Poincaré-Einstein (or ambient) metric. In turn, the Taylor coefficients of the square root $w(r)$ easily can be described in terms of the holographic coefficients $v_{2 j}$. 
In the locally conformally flat case, Theorem 9.1 extends to all $Q$-curvatures $Q_{2 N}$. In fact, in the proof it suffices to replace Theorem 8.3 by Theorem 8.4 . Moreover, in that case, the evaluation of Theorem 9.1 is greatly simplified by the closed formula

$$
w(r)=\sqrt{\operatorname{det}\left(1-r^{2} / 2 \mathrm{P}\right)} .
$$

Some information on special contributions to $Q_{2 N}$ can be easily deduced from Theorem 9.1. In fact, using

$$
n_{(N)}=n_{(1, \ldots, 1)}=1,
$$

Eq. (9.1) shows that $Q_{2 N}$ is of the form

$$
(-1)^{N} N !(N-1) ! 2^{2 N} w_{2 N}+\cdots+4(-1)^{N} \mathcal{M}_{2}^{N-1}\left(w_{2}\right) .
$$

Hence, by $4 w_{2}=-\mathrm{J}$, we recover the well-known fact ([B95], Corollary 1.5) that the quantity $Q_{2 N}$ contains the term $(-1)^{N} \Delta^{N-1} \mathrm{~J}$. This is the contribution with the most derivatives. Moreover, using

$$
n_{(N-1,1)}=N-1
$$

Theorem 9.1 implies that for $N \geq 3$ the full tensor $g_{(2 N-4)}$ contributes to $Q_{2 N}$ through

$$
(-1)^{N-1}(N-1) !(N-2) ! 2^{2 N-4} \delta\left(g_{(2 N-4)} d \mathrm{~J}\right) .
$$

We also reproduce the result ([B95], Corollary 1.6) that the contribution with the most derivatives to the total $Q$-curvature

$$
\int_{M} Q_{2 N} d v o l
$$

on the closed manifold $M$ has the form

$$
\left(\frac{n}{2}-N\right) \int_{M}\left(\nabla^{N-2} \mathrm{~J}, \nabla^{N-2} \mathrm{~J}\right) d v o l .
$$

In fact, Theorem 9.1 implies that these contributions only come from

$$
n_{(1, \ldots, 1)} 2^{2} \mu_{2} \mathcal{M}_{2}^{N-2}\left(w_{2}\right)+n_{(2,1, \ldots, 1)} 2^{2} \mu_{4} \mathcal{M}_{2}^{N-3}\left(w_{2}\right) .
$$

But using (9.6) and

$$
n_{(1, \ldots, 1)}=1 \quad \text { and } \quad n_{(2,1, \ldots, 1)}=N-1 \text {, }
$$

these terms yield

$$
\left(\frac{n}{2}-1\right) \int_{M} \mathrm{~J} \Delta^{N-2}(\mathrm{~J}) d v o l-(N-1) \int_{M} \Delta(\mathrm{J}) \Delta^{N-3}(\mathrm{~J}) d v o l .
$$

Now partial integration gives (9.3).

Finally, we illustrate Theorem 9.1 by the following three low-order special cases.

Example 9.1. For $N=2$, Theorem 9.1 states that

$$
Q_{4}=32 w_{4}+4 \mathcal{M}_{2}\left(w_{2}\right) .
$$

In view of

$$
4 w_{2}=-\mathrm{J} \text { and } 32 w_{4}=\mathrm{J}^{2}-2|\mathrm{P}|^{2},
$$

this formula is equivalent to the recursive formula

$$
Q_{4}=32 w_{4}-P_{2}\left(Q_{2}\right)
$$


and the common explicit formula

$$
Q_{4}=\frac{n}{2} \mathrm{~J}^{2}-2|\mathrm{P}|^{2}-\Delta \mathrm{J}
$$

Example 9.2. The following two formulas are the universal recursive formula of [J10b] and the explicit formula of Theorem 9.1 for $Q_{6}$ :

$$
Q_{6}+2 P_{2}\left(Q_{4}\right)-2 P_{4}\left(Q_{2}\right)+3 P_{2}^{2}\left(Q_{2}\right)=-3 ! 2 ! 2^{6} w_{6}
$$

and

$$
Q_{6}=-3 ! 2 ! 2^{6} w_{6}-64 \mathcal{M}_{2}\left(w_{4}\right)-8 \mathcal{M}_{4}\left(w_{2}\right)-4 \mathcal{M}_{2}^{2}\left(w_{2}\right) .
$$

In both formulas, we have collected all terms of the same nature on the same side. In order to illustrate the effectiveness of (9.5), we make the terms fully explicit. We have

with

$$
\mathcal{M}_{2}=P_{2}=\Delta-\left(\frac{n}{2}-1\right) \mathrm{J} \quad \text { and } \quad \mathcal{M}_{4}=-4 \delta(\mathrm{P} d)+\mathcal{M}_{4}(1)
$$

i.e.,

$$
\mathcal{M}_{4}(1)+4 \mathcal{M}_{2}\left(w_{2}\right)=32\left(\frac{n}{2}-2\right) w_{4},
$$

$$
\begin{aligned}
\mathcal{M}_{4}(1) & =\left(\frac{n}{2}-2\right)\left(\mathrm{J}^{2}-2|\mathrm{P}|^{2}\right)+\left(\Delta-\left(\frac{n}{2}-1\right) \mathrm{J}\right)(\mathrm{J}) \\
& =-\mathrm{J}^{2}-(n-4)|\mathrm{P}|^{2}+\Delta \mathrm{J} .
\end{aligned}
$$

Thus, by a straightforward calculation, (9.5) gives

$$
\begin{aligned}
Q_{6}=-3 ! 2 ! 2^{6} w_{6}-\left(\frac{n}{2}+1\right) \Delta\left(\mathrm{J}^{2}\right)+4 \Delta & \left(|\mathrm{P}|^{2}\right)-8 \delta(\mathrm{P} d \mathrm{~J})+\Delta^{2} \mathrm{~J} \\
& -\left(\frac{n}{2}-3\right) \mathrm{J} \Delta \mathrm{J}-4(n-3) \mathrm{J}|\mathrm{P}|^{2}+\frac{n^{2}-12}{4} \mathrm{~J}^{3}
\end{aligned}
$$

or, equivalently,

$$
\begin{aligned}
Q_{6}=-3 ! 2 ! 2^{5} v_{6}-\left(\frac{n}{2}+1\right) & \Delta\left(\mathrm{J}^{2}\right)+4 \Delta\left(|\mathrm{P}|^{2}\right)-8 \delta(\mathrm{P} d \mathrm{~J})+\Delta^{2} \mathrm{~J} \\
& -\left(\frac{n}{2}-3\right) \mathrm{J} \Delta \mathrm{J}-4(n-6) \mathrm{J}|\mathrm{P}|^{2}+\frac{(n-6)(n+6)}{4} \mathrm{~J}^{3} .
\end{aligned}
$$

In the last step we have used (10.4) and

$$
16 w_{6}+8 v_{2} w_{4}=8 v_{6} .
$$

The identity (9.7) is equivalent to Theorem 6.10.3 in [J09a]. The same formula for $Q_{6}$ follows from (9.4) by a somewhat longer calculation. Note that (9.7) also reproduces the familiar fact that, in dimension $n=6, Q_{6}$ differs from a multiple of $v_{6}$ by a divergence. In that case, we actually find that

$$
Q_{6}=-3 ! 2 ! 2^{5} v_{6}-64 \mathcal{M}_{2}^{0}\left(w_{4}\right)-8 \mathcal{M}_{4}^{0}\left(w_{2}\right)-4 \mathcal{M}_{2}^{0} \mathcal{M}_{2}\left(w_{2}\right) .
$$

Example 9.3. For even $n \geq 8$ and odd $n \geq 3$ and any metric,

$$
\begin{aligned}
Q_{8}=3 ! 4 ! 2^{8} w_{8} & +2^{2} 3 \mathcal{M}_{6}\left(w_{2}\right)+2^{4} 18 \mathcal{M}_{4}\left(w_{4}\right)+2^{6} 36 \mathcal{M}_{2}\left(w_{6}\right) \\
& +2^{2} 3 \mathcal{M}_{4} \mathcal{M}_{2}\left(w_{2}\right)+2^{2} 4 \mathcal{M}_{2} \mathcal{M}_{4}\left(w_{2}\right)+2^{4} 6 \mathcal{M}_{2}^{2}\left(w_{4}\right)+2^{2} \mathcal{M}_{2}^{3}\left(w_{2}\right) .
\end{aligned}
$$


The operators $\mathcal{M}_{2}, \mathcal{M}_{4}, \mathcal{M}_{6}$ are second-order (see (10.7), (10.8)). The quantity $w_{8}$ is given by (10.22) and (10.23). This formula should be compared with the universal recursive formula

$$
\begin{aligned}
Q_{8}+3 P_{2}\left(Q_{6}\right) & +3 P_{6}\left(Q_{2}\right)-9 P_{4}\left(Q_{4}\right) \\
& -8 P_{2} P_{4}\left(Q_{2}\right)+12 P_{2}^{2}\left(Q_{4}\right)-12 P_{4} P_{2}\left(Q_{2}\right)+18 P_{2}^{3}\left(Q_{2}\right)=3 ! 4 ! 2^{8} w_{8}
\end{aligned}
$$

proved in $\mathrm{J} 09 \mathrm{c}$, J10b].

Another formula for $Q_{8}$ was displayed in GoP03 (see Fig. 5). Unfortunately, it is a rather non-trivial task to compare that formula with those given in Example 9.39

\section{SOME LOW-ORDER CASES}

In the present section, we make explicit the consequences of the general theory for the third and the fourth GJMS-operator $P_{6}$ and $P_{8}$.

10.1. The conformal cube $P_{6}$ of the Laplacian. We discuss two formulas for the conformally covariant cube $P_{6}$ of the Laplacian. In this case, explicit formulas in terms of the metric are still not too complicated. Although a number of alternative approaches have been used to understand this higher-order generalization of the Paneitz-operator, it seems that the formula for $P_{6}$ given in Theorem 10.2 has not been derived before. For alternative methods we refer to [B85, W86], W00], GoP03] and CGS08.

We recall that one of the key observations which leads to the following results is the commutator relation

$$
\mathcal{M}_{6} i^{*}=4\left(i^{*}\left[\bar{P}_{2}, \overline{\mathcal{M}}_{4}\right]-\left[P_{2}, \mathcal{M}_{4}\right] i^{*}\right) .
$$

By $\mathcal{M}_{4}=P_{4}-P_{2}^{2}$ (for any metric), the latter relation is equivalent to the identity

$$
\mathcal{M}_{6} i^{*}=4\left(i^{*}\left[\bar{P}_{2}, \bar{P}_{4}\right]-\left[P_{2}, P_{4}\right] i^{*}\right) .
$$

In the critical dimension $n=6$ and for locally conformally flat metrics, this relation first appeared in [J09a] (Theorem 6.11.17).

10.1.1. The recursive formula. The following formula describes $P_{6}$ in terms of the lower-order GJMS-operators $P_{2}, P_{4}$ and the $Q$-curvatures $Q_{6}$. For all metrics and in dimension $n \geq 3(n \neq 4)$, we have

$$
P_{6}=\left(2 P_{2} P_{4}+2 P_{4} P_{2}-3 P_{2}^{3}\right)^{0}-48 \delta\left(\mathrm{P}^{2} d\right)-\frac{16}{n-4} \delta(\mathcal{B} d)-\left(\frac{n}{2}-3\right) Q_{6} .
$$

Here the tensor

$$
\mathcal{B}_{i j} \stackrel{\text { def }}{=} \Delta(\mathrm{P})_{i j}-\nabla^{k} \nabla_{j}(\mathrm{P})_{i k}+\mathrm{P}^{k l} W_{k i j l}
$$

being defined by the Schouten tensor $\mathrm{P}$ and the Weyl tensor $W$ is known as the Bach tensor. As usual, the superscript ${ }^{0}$ removes the zeroth-order term. Eq. (10.1) is equivalent to the formula

$$
\mathcal{M}_{6}^{0}=-48 \delta\left(\mathrm{P}^{2} d\right)-\frac{16}{n-4} \delta(\mathcal{B} d)
$$

\footnotetext{
${ }^{9}$ In this direction, we only note that all formulas contain the contribution $-96\left(\Omega^{(1)}, \Omega^{(1)}\right)$ with a second-order pole at $n=4$.
} 
for the principal part of $\mathcal{M}_{6}$ (see also the discussion in Section 7.1). Finally, the $Q$-curvature $Q_{6}$ is given by the universal recursive formula

$$
Q_{6}+2 P_{2}\left(Q_{4}\right)-2 P_{4}\left(Q_{2}\right)+3 P_{2}^{2}\left(Q_{2}\right)=-3 ! 2 ! 2^{6} w_{6}
$$

(see Eq. (9.4) ), where the quantity $w_{6}$ is related through

$$
16 w_{6}=8 v_{6}-4 v_{4} v_{2}+v_{2}^{3}
$$

to the holographic coefficients

$$
v_{2}=-\frac{1}{2} \mathrm{~J}, \quad v_{4}=\frac{1}{8}\left(\mathrm{~J}^{2}-|\mathrm{P}|^{2}\right) \quad \text { and } \quad v_{6}=-\frac{1}{8} \operatorname{tr}\left(\wedge^{3} \mathrm{P}\right)-\frac{1}{24(n-4)}(\mathcal{B}, \mathrm{P}) .
$$

The resulting fully explicit formula for $Q_{6}$ is displayed in (9.7).

Alternatively, by combining the definition of $\mathcal{M}_{6}$ (see Eq. (12.3)) with (10.3), we obtain the recursive formula

$$
P_{6}=\left(2 P_{2} P_{4}+2 P_{4} P_{2}-3 P_{2}^{3}\right)-48 \delta\left(\mathrm{P}^{2} d\right)-\frac{16}{n-4} \delta(\mathcal{B} d)+\mu_{6}
$$

with the scalar curvature quantity $\mu_{6}$ as in (10.13).

10.1.2. The explicit formula. Under the same assumptions on the dimension $n$ as in Section 10.1.1, the following formula describes $P_{6}$ for general metrics in terms of the second-order operators $\mathcal{M}_{2}, \mathcal{M}_{4}$ and $\mathcal{M}_{6}$. We have

$$
P_{6}=\mathcal{M}_{6}+2 \mathcal{M}_{2} \mathcal{M}_{4}+2 \mathcal{M}_{4} \mathcal{M}_{2}+\mathcal{M}_{2}^{3}
$$

(see Example 2.2), where

$$
\mathcal{M}_{2}=P_{2}=-\delta d+\mu_{2}, \quad \mathcal{M}_{4}=-4 \delta(\mathrm{P} d)+\mu_{4}
$$

and

$$
\mathcal{M}_{6}=-48 \delta\left(\mathrm{P}^{2} d\right)-\frac{16}{n-4} \delta(\mathcal{B} d)+\mu_{6} .
$$

The curvature quantities $\mu_{2}, \mu_{4}$ and $\mu_{6}$ are determined by the identities

$$
\mu_{2}=2^{2}\left(\frac{n}{2}-1\right) w_{2}, \quad \mu_{4}=2 ! 2^{4}\left(\frac{n}{2}-2\right) w_{4}-4 \mathcal{M}_{2}\left(w_{2}\right)
$$

and

$$
\mu_{6}=3 ! 2 ! 2^{6}\left(\frac{n}{2}-3\right) w_{6}-4^{2} \mathcal{M}_{4}\left(w_{2}\right)-8^{2} \mathcal{M}_{2}\left(w_{4}\right) .
$$

These are special cases of Theorem 8.3 (see also (8.8) and (8.12)). Note also that

$$
2 w_{2}=v_{2} \quad \text { and } \quad 8 w_{4}=4 v_{4}-v_{2}^{2} .
$$

Some calculations yield the explicit formulas

$$
\mu_{4}=-\mathrm{J}^{2}-(n-4)|\mathrm{P}|^{2}+\Delta \mathrm{J} \quad(\text { see Eq. }(\underline{9.6}))
$$

and

$$
\begin{aligned}
\mu_{6}=-8 \frac{n-6}{n-4}(\mathcal{B}, \mathrm{P})-8(n-6) \operatorname{tr} & \left(\mathrm{P}^{3}\right) \\
& -16 \mathrm{~J}|\mathrm{P}|^{2}-4|d \mathrm{~J}|^{2}+4 \Delta\left(|\mathrm{P}|^{2}\right)-16 \delta(\mathrm{P} d \mathrm{~J}) .
\end{aligned}
$$

The formulas (10.12) and (10.13) should also be compared with Theorem 8.5 . 
Now we use these results to derive a fully explicit formula for $P_{6}$ in terms of the metric. In the critical case, we find the following result.

Theorem 10.1. On manifolds of dimension $n=6$, the GJMS-operator $P_{6}$ is given by the explicit formula

$$
P_{6}=\Delta^{3}+4 \Delta \delta T_{2} d+4 \delta T_{2} d \Delta+2 \Delta(\mathrm{J} \Delta)+8 \delta T_{4} d
$$

with

$$
T_{2}=\mathrm{J}-2 \mathrm{P} \quad \text { and } \quad T_{4}=-\mathrm{J}^{2}+|\mathrm{P}|^{2}+4 \mathrm{JP}-6 \mathrm{P}^{2}-\mathcal{B} .
$$

Theorem 10.1 extends as follows to general dimensions.

Theorem 10.2. On manifolds of dimension $n \geq 3(n \neq 4)$, the GJMS-operator $P_{6}$ is given by the explicit formula

$$
P_{6}=\Delta^{3}+\Delta \delta T_{2} d+\delta T_{2} d \Delta+\left(\frac{n}{2}-1\right) \Delta(\mathrm{J} \Delta)+\delta T_{4} d-\left(\frac{n}{2}-3\right) Q_{6}
$$

with

$$
T_{2}=(n-2) \mathrm{J}-8 \mathrm{P}
$$

$$
\begin{aligned}
T_{4}=-\left(3\left(\frac{n}{2}-1\right)^{2}-4\right) \mathrm{J}^{2}+4(n-4)|\mathrm{P}|^{2}+16\left(\frac{n}{2}-1\right) \mathrm{JP} & \\
& +(n-6) \Delta \mathrm{J}-48 \mathrm{P}^{2}-\frac{16}{n-4} \mathcal{B}
\end{aligned}
$$

and $Q_{6}$ as in (9.7).

Proof. We make (10.6) explicit. We recall that

$$
\mu_{2}=-\left(\frac{n}{2}-1\right) \mathrm{J} \quad \text { and } \quad \mu_{4}=-\mathrm{J}^{2}-(n-4)|\mathrm{P}|^{2}+\Delta \mathrm{J}
$$

(see Eq. (10.12) ). In order to prove the assertion, it suffices to determine the nonzero order contributions. Let $\lambda=\frac{n}{2}-1$. Then we have to determine the sum of the non-zero order terms of the operators

$$
\begin{aligned}
& \Delta^{3} u-\lambda \mathrm{J} \Delta^{2} u-\lambda \Delta^{2}(\mathrm{~J} u)-\lambda \Delta(\mathrm{J} \Delta u)+\lambda^{2} \mathrm{~J} \Delta(\mathrm{J} u)+\lambda^{2} \mathrm{~J}^{2} \Delta u+\lambda^{2} \Delta\left(\mathrm{J}^{2} u\right), \\
& -2 \Delta\left(\mathrm{J}^{2} u\right)-2 \mathrm{~J}^{2} \Delta u+2 \Delta(u \Delta \mathrm{J})+2 \Delta(\mathrm{J}) \Delta(u) \\
& -8 \Delta \delta(\mathrm{P} d u)-8 \delta(\mathrm{P} d) \Delta u+4(n-4) \delta\left(|\mathrm{P}|^{2} d u\right)-48 \delta\left(\mathrm{P}^{2} d u\right)-8 \delta(\mathcal{B} d u)
\end{aligned}
$$

and

$$
8 \lambda \mathrm{J} \delta(\mathrm{P} d u)+8 \lambda \delta(\mathrm{P} d(\mathrm{~J} u))
$$

In the following calculations, we omit the zeroth-order terms. Now

$$
\begin{aligned}
\mathrm{J} \Delta(\mathrm{J} u) & =\mathrm{J}(\Delta(\mathrm{J}) u+2(d \mathrm{~J}, d u)+\mathrm{J} \Delta(u)) \\
& =\left(d \mathrm{~J}^{2}, d u\right)+\mathrm{J}^{2} \Delta(u) \\
& =-\delta\left(\mathrm{J}^{2} d u\right)
\end{aligned}
$$

and

$$
\mathrm{J}^{2} \Delta(u)+\Delta\left(\mathrm{J}^{2} u\right)=-2 \delta\left(\mathrm{J}^{2} d u\right)
$$

imply that the last three terms of (10.16) yield the contribution

$$
-3 \lambda^{2} \delta\left(\mathrm{J}^{2} d u\right) \text {. }
$$


Similarly, the first two terms of (10.17) yield the contribution

$$
4 \delta\left(\mathrm{J}^{2} d u\right) \text {. }
$$

Next, we apply the relation $\Delta(d \mathrm{~J}, d u)=-\Delta \delta(\mathrm{J} d u)-\Delta(\mathrm{J} \Delta u)$ to find that

$$
\begin{aligned}
& \mathrm{J} \Delta^{2} u+\Delta^{2}(\mathrm{~J} u)+\Delta(\mathrm{J} \Delta u) \\
& =\mathrm{J} \Delta^{2} u+\Delta(\Delta(\mathrm{J}) u+2(d \mathrm{~J}, d u)+\mathrm{J} \Delta(u))+\Delta(\mathrm{J} \Delta u) \\
& =\mathrm{J} \Delta^{2} u+2(d \Delta \mathrm{J}, d u)+\Delta(\mathrm{J}) \Delta(u)-2 \Delta \delta(\mathrm{J} d u) .
\end{aligned}
$$

It follows that the first four terms in (10.16) and the last two terms of (10.17) give rise to the contribution

$$
\begin{array}{r}
\Delta^{3} u-\left(\frac{n}{2}-1\right) \mathrm{J} \Delta^{2} u-(n-2)(d \Delta \mathrm{J}, d u)-\left(\frac{n}{2}-1\right) \Delta(\mathrm{J}) \Delta(u)+(n-2) \Delta \delta(\mathrm{J} d u) \\
+4 \Delta(u) \Delta(\mathrm{J})+4(d u, d \Delta \mathrm{J}) .
\end{array}
$$

We rewrite this sum in the form

$$
\begin{aligned}
\Delta^{3} u-\left(\frac{n}{2}-1\right)\left(\mathrm{J} \Delta^{2} u-\Delta(\mathrm{J}) \Delta(u)\right)+(n-2) \Delta & \delta(\mathrm{J} d u) \\
& -(n-6)((d \Delta \mathrm{J}, d u)+\Delta(\mathrm{J}) \Delta(u)) .
\end{aligned}
$$

Now the identity

$$
-\left(\frac{n}{2}-1\right) \mathrm{J} \Delta^{2} u+\left(\frac{n}{2}-1\right) \Delta(\mathrm{J}) \Delta(u)=(n-2) \delta(\mathrm{J} d \Delta u)+\left(\frac{n}{2}-1\right) \Delta(\mathrm{J} \Delta u)
$$

implies that the sum equals

$$
\Delta^{3} u+(n-2) \delta(\mathrm{J} d \Delta u)+(n-2) \Delta \delta(\mathrm{J} d u)+\left(\frac{n}{2}-1\right) \Delta(\mathrm{J} \Delta u)+(n-6) \delta(\Delta \mathrm{J}, d u) .
$$

Finally, we observe that (10.18) equals $16 \lambda \delta(\mathrm{JP} d u)$ (modulo a zeroth-order term). This completes the proof.

Some comments are in order. First of all, an easy calculation confirms that for the round sphere $\mathbb{S}^{n}$ the formula (10.15) actually simplifies to the product

$$
(\Delta-m(m-1))(\Delta-(m+1)(m-2))(\Delta-(m+2)(m-3)), m=n / 2 .
$$

The first four terms in (10.15) cover all contributions to $P_{6}$ which contain more than four derivatives. In Subsection 11.7 we shall recognize this fact as a special case of a general result.

Next, it is worth noting that for general metrics the formula (10.14) coincides with a formula of Branson for a conformally covariant cube of the Laplacian in dimension $n=6$ ([B85, Theorem 2.8) 10 Another formula for a conformally covariant cube of the Laplacian in general dimensions $n \geq 6$ containing the second-order operator $-\delta(\mathcal{B} d) /(n-4)$ was given in Proposition 3.1 of [W86]. However, a full comparison is difficult since Wünsch's formula is stated in a less explicit form. Finally, we note that Theorem 10.1 reproduces the formula for the critical $P_{6}$ contained in Proposition 8.3 of AG11] 11

\footnotetext{
${ }^{10}$ Branson's tensor $Y$ actually coincides with the sum $-\mathcal{B}-(n-4) \mathrm{P}^{2}$.

${ }^{11}$ In AG11, P denotes the double of the Schouten tensor. Thus, in terms of the notation of the present paper, the tensors $E_{1}$ and $E_{2}$ in [AG11] are given by $2 \mathrm{P}-\mathrm{J}$ and $4 \mathrm{P}^{2}+2 \mathcal{B}-2|\mathrm{P}|^{2}$.
} 
An independent proof of the conformal covariance of the operator on the righthand side of (10.1) in the critical dimension $n=6$ can be found in the Appendix 13.2 of [J09b. In general dimensions, the formula (10.1) first appeared in Section 6.12 of [J09a. There it was derived by (infinitesimal) conformal variation of (a version of) the universal recursive formula (9.4) for $Q_{6}$.

10.2. The conformal fourth power $P_{8}$ of the Laplacian. Explicit formulas for the principal parts of $\mathcal{M}_{4}$ and $\mathcal{M}_{6}$ are displayed in Section 10.1. The following result follows from Theorem 7.1. It leads to an explicit formula for $P_{8}$.

Theorem 10.3. For even $n \geq 8$ and odd $n \geq 3$,

$$
\mathcal{M}_{8}^{0}=-96 \delta\left(\left[\Omega^{(2)}-4\left(\mathrm{P} \Omega^{(1)}+\Omega^{(1)} \mathrm{P}\right)+12 \mathrm{P}^{3}\right] d\right),
$$

where $\Omega^{(1)}, \Omega^{(2)}$ are the first two extended obstruction tensors.

For the definition of extended obstruction tensors we refer to [G09] and (12.20). Section 2 of G09] also gives an explicit formula for $\Omega^{(2)}$ in terms of $g$. The full details of the derivation of (10.19) from the general formula for the principal part can be found in $[\mathrm{J09b}$.

Similarly as in Subsection 10.1.1, the equation (10.19) can be viewed as a recursive formula for $P_{8}$. We omit the details and continue with the description of $P_{8}$ in terms of the operators $\mathcal{M}_{2}, \ldots, \mathcal{M}_{8}$. By combining Theorem 10.3 with Example 2.3, we obtain the following result.

Theorem 10.4. For all even $n \geq 8$ and all odd $n \geq 3$, the GJMS-operator $P_{8}$ is given by the formula

$$
\begin{array}{r}
P_{8}=\mathcal{M}_{2}^{4}+3\left(\mathcal{M}_{2}^{2} \mathcal{M}_{4}+\mathcal{M}_{4} \mathcal{M}_{2}^{2}\right)+4 \mathcal{M}_{2} \mathcal{M}_{4} \mathcal{M}_{2}+9 \mathcal{M}_{4}^{2}+3\left(\mathcal{M}_{2} \mathcal{M}_{6}+\mathcal{M}_{6} \mathcal{M}_{2}\right) \\
-96 \delta\left(\left[\Omega^{(2)}-4\left(\mathrm{P} \Omega^{(1)}+\Omega^{(1)} \mathrm{P}\right)+12 \mathrm{P}^{3}\right] d\right)+\mu_{8},
\end{array}
$$

where

$$
\mu_{8}=\left(\frac{n}{2}-4\right) 3 ! 4 ! 2^{8} w_{8}-48^{2} \mathcal{M}_{2}\left(w_{6}\right)-24^{2} \mathcal{M}_{4}\left(w_{4}\right)-6^{2} \mathcal{M}_{6}\left(w_{2}\right) .
$$

The formula (10.21) is a consequence of Theorem 8.3. In terms of holographic coefficients, the function $w_{8}$ can be described as

$$
128 w_{8}=64 v_{8}-32 v_{6} v_{2}-16 v_{4}^{2}+24 v_{2}^{2} v_{4}-5 v_{2}^{4},
$$

where $v_{2}, v_{4}, v_{6}$ are as in Section 10.1. Finally, $v_{8}$ is given by

$$
\begin{aligned}
2^{4} v_{8}= & \operatorname{tr}\left(\wedge^{4} \mathrm{P}\right) \\
& +\frac{1}{3} \operatorname{tr}\left(\mathrm{P}^{2} \Omega^{(1)}\right)-\frac{1}{3} \operatorname{tr}(\mathrm{P}) \operatorname{tr}\left(\mathrm{P} \Omega^{(1)}\right)-\frac{1}{12} \operatorname{tr}\left(\mathrm{P} \Omega^{(2)}\right)-\frac{1}{12} \operatorname{tr}\left(\Omega^{(1)} \Omega^{(1)}\right)
\end{aligned}
$$

(see [G09], Eq. (2.23)).

The above results actually suffice to derive from (10.20) a fully explicit formula for $P_{8}$ in terms of the metric. The details will be presented elsewhere. Here we only note that the contributions with more than four derivatives are contained in the sum

$$
\sum_{k=0}^{2} \Delta^{k} \delta((n-2) \mathrm{J}-4(k+1)(3-k) \mathrm{P}) d \Delta^{2-k}+\left(\frac{n}{2}-1\right) \sum_{k=1}^{2} \Delta^{k}\left(\mathrm{~J} \Delta^{3-k}\right) .
$$


This is a special case of a result discussed in Subsection 11.7.

We finish the present section with two brief comments on alternative approaches. As special cases of their approach using tractor calculus, Gover and Peterson GoP03] derived formulas for $P_{6}$ and $P_{8}$ in terms of the metric (and in general dimensions). Unfortunately, the conventions used in the formulation of their final results hinder a direct comparison with the present results. Wünsch W00] (Theorem 4.3) gave a formula for a conformally covariant fourth power of the Laplacian in general dimensions. That formula is far from being explicit in terms of the metric and the relation to the above description of the operator $P_{8}$ (see Theorem 10.4) is not clear 12

\section{FURTHER RESULTS AND COMMENTS}

In the present section, we describe a few additional results and collect a number of comments.

11.1. General signature. Although the original construction of GJMS-operators works for metrics of arbitrary signature $(p, q)$, we restricted all discussions in the present paper to the framework of Riemannian metrics. This is motivated by our usage of the identification of GJMS-operators in the asymptotic expansions of eigenfunctions of the Laplacian of Poincaré-Einstein metrics [GZ03] and of the theory of residue families J09a. However, it is natural to expect that the main results literally extend to metrics of arbitrary signature.

For the discussion of the special cases $\mathbb{S}^{(p, q)}$ we refer to [J09b] and [JK09] (see also Example 8.2). Another interesting special case in this direction are $p p$-waves

$$
g=\sum_{i=1}^{n-2} d x_{i}^{2}+2 d u d r+2 h d u^{2}
$$

with $h \in C^{\infty}\left(\mathbb{R}^{n}\right)$ being independent of $r$. For these metrics, Leistner and Nurowski [LN09] found a closed formula for the corresponding ambient metrics. In particular, one finds that $v(r)=1$. Therefore, the extension of (1.9) reduces to the simple formula $\mathcal{H}(r)=-\delta\left(g_{r}^{-1} d\right)$. It follows that (for $N \geq 2$ ) the operators $\mathcal{M}_{2 N}$ are respective constant multiples of $\Delta^{N-1}(h)\left(\partial^{2} / \partial r \partial u\right)$.

We also note that the validity of all factorizations of residue families for general metrics (Theorem 3.1 and Theorem 3.2) has the consequence that the results and conjectures in [FJ] literally extend from locally conformally flat metrics to general metrics.

11.2. Einstein metrics. If $g$ is Einstein, Theorem 1.1 implies Gover's product formula Go06]

$$
P_{2 N}(g)=\prod_{j=\frac{n}{2}}^{\frac{n}{2}+N-1}\left(\Delta_{g}-\lambda_{g} j(n-1-j)\right)
$$

\footnotetext{
${ }^{12}$ Note only that the contributions of $\left|\Omega^{(1)}\right|^{2}$ to both operators differ.
} 
where $\lambda_{g}$ is the constant $\operatorname{scal}(g) / n(n-1)$. Eq. (11.1) extends the product formula

$$
P_{2 N}=\prod_{j=\frac{n}{2}}^{\frac{n}{2}+N-1}(\Delta-j(n-1-j))
$$

for the round sphere $\mathbb{S}^{n}$. We sketch the argument. First, we recall that [FG07]

$$
g_{+}=r^{-2}\left(d r^{2}+\left(1-\lambda r^{2} / 4\right)^{2} g\right) .
$$

Hence $w(r)=\left(1-\lambda r^{2} / 4\right)^{\frac{n}{2}}$ and a calculation shows that

$$
\mathcal{D}(g)(r)=\left(1-\lambda \frac{r^{2}}{4}\right)^{-2}\left(\Delta-\lambda \frac{n}{2}\left(\frac{n}{2}-1\right)\right) .
$$

Now Theorem 1.1 yields

$$
\mathcal{H}(g)(r)=\left(1-\lambda r^{2} / 4\right)^{-2} P_{2}(g)
$$

Hence

$$
\mathcal{M}_{2 N}=N !(N-1) ! \lambda^{N-1} P_{2}
$$

It follows that in the sum

$$
P_{2 N}=\sum_{|I|=N} n_{I} \mathcal{M}_{2 I}
$$

the terms with exactly $r$ factors are multiples of $\lambda^{N-r} P_{2}^{r}$. Now on the sphere $\mathbb{S}^{n}$ the sum on the right-hand side of (11.2) simplifies to the product

$$
\prod_{k=0}^{N-1}\left(P_{2}+k(k+1)\right) \text {. }
$$

This follows by combining Theorem 2.1 with the summation formula Theorem 6.1 in $\mathrm{J} 09 \mathrm{~b}$. But an easy modification of the proof of the latter result yields

$$
\prod_{k=0}^{N-1}\left(P_{2}+\lambda k(k+1)\right)
$$

in the present situation. This proves (11.1).

11.3. Singular cases. We note that the formula (10.6) implies the conformal covariance of the right-hand side if $4 \neq n \geq 3$. Moreover, it shows that in dimension $n=4$ the residue

$$
R_{6}=\operatorname{Res}_{n=4}\left(\mathcal{M}_{6}\right)
$$

is conformally covariant in the sense that $e^{5 \varphi} \hat{R}_{6}=R_{6} e^{-\varphi} 13$ A calculation using (10.8), (10.10) and (10.4) actually yields the explicit formula

$$
R_{6}=-16 \delta(\mathcal{B} d)+\operatorname{Res}_{n=4}\left(\mu_{6}\right)=-16(\delta(\mathcal{B} d)-(\mathcal{B}, \mathrm{P})) .
$$

Similarly, the formula for $P_{8}$ in Example 2.3 implies the conformal covariance of the right-hand side if $n \geq 3$ differs from 4 and 6 . Moreover, it shows that in dimension $n=6$ the residue

$$
R_{8}=\operatorname{Res}_{n=6}\left(\mathcal{M}_{8}\right)
$$

\footnotetext{
${ }^{13}$ Here the dimension $n$ is treated as a formal variable.
} 
is conformally covariant, i.e., $e^{7 \varphi} \hat{R}_{8}=R_{8} e^{-\varphi}$. In order to find an explicit formula for $R_{8}$, we first note that

$$
\mathcal{M}_{8}^{0}=-96 \delta\left(\Omega^{(2)} d\right)+\cdots
$$

implies

$$
\operatorname{Res}_{n=6}\left(\mathcal{M}_{8}\right)=-48 \delta\left(\mathcal{O}_{6} d\right)+\operatorname{Res}_{n=6}\left(\mu_{8}\right),
$$

where

$$
\mathcal{O}_{6}=2 \operatorname{Res}_{n=6}\left(\Omega^{(2)}\right)
$$

(see [G09], Proposition 2.8) is the Fefferman-Graham obstruction tensor in dimension $n=6$. An explicit formula for $\mathcal{O}_{6}$ can be found, for instance, in GH05]. Now Theorem 8.3 and

$$
v_{8}=-\frac{1}{2^{4}} \frac{1}{12} \operatorname{tr}\left(\mathrm{P} \Omega^{(2)}\right)+\cdots
$$

(see Eq. (10.23) ) yield the explicit formula

$$
R_{8}=-48\left(\delta\left(\mathcal{O}_{6} d\right)-\left(\mathcal{O}_{6}, \mathrm{P}\right)\right)
$$

As the Bach tensor $\mathcal{B}$ in dimension $4, \mathcal{O}_{6}$ is trace-free and divergence-free. Moreover, its transformation law $e^{4 \varphi} \hat{\mathcal{O}}_{6}=\mathcal{O}_{6}$ in dimension six generalizes $e^{2 \varphi} \hat{\mathcal{B}}=\mathcal{B}$ in dimension four. A direct calculation, using these properties, confirms that the operator $R_{8}$ is conformally covariant (in dimension six).

We also note that $\mathcal{M}_{8}$ has a second-order pole at $n=4$. It is caused by the contribution $\left|\Omega^{(1)}\right|^{2}$ to the holographic coefficient $v_{8}$ which in turn contributes to the zeroth-order term $\mu_{8}=\mathcal{M}_{8}(1)$ of $\mathcal{M}_{8}$. The corresponding scalar quantity $I=|\mathcal{B}|^{2}$ is conformally covariant (in dimension $n=4$ ) in the sense that $e^{8 \varphi} \hat{I}=I$.

11.4. More universal relations for GJMS-operators. We recall that the degree of the polynomial $\lambda \mapsto D_{2 N}^{r e s}(\lambda)$ is $N$. Thus, Theorem 4.1 implies the vanishing results

$$
d_{2 N}^{(1)}=\cdots=d_{2 N}^{(N-1)}=0
$$

for the coefficients of $D_{2 N}(\lambda)$ (see Eq. (4.6) ). These are $N-1$ universal identities which involve the GJMS-operators $P_{2}, \ldots, P_{2 N}$ and $\bar{P}_{2}, \ldots, \bar{P}_{2 N}$. Only the first two identities

$$
d_{2 N}^{(1)}=0 \quad \text { and } \quad d_{2 N}^{(2)}=0
$$

were utilized in the proofs of the main results of the present paper. In that connection, it was important to recognize the equivalence of the identities in (11.4) to the restriction property and the commutator relations for the operators $\mathcal{M}_{2 N}$ (using the restriction property of all $\mathcal{M}_{2 M}$ for $M<N$.) A similar description of the identities $d_{2 N}^{(k)}=0$ for $k=3, \ldots, N-1$ (for $N \geq 4$ ) and an understanding of their significance remain to be found. Along these lines, one can prove for instance that the three relations in (11.3) for $N=4$ contain the additional identity

$$
\left(P_{2} \mathcal{M}_{6}+3 \mathcal{M}_{6} P_{2}-4\left[P_{2},\left[P_{2}, \mathcal{M}_{4}\right]\right]\right) i^{*}=i^{*}\left(\overline{\mathcal{M}}_{6} \bar{P}_{2}+3 \bar{P}_{2} \overline{\mathcal{M}}_{6}-4\left[\bar{P}_{2},\left[\bar{P}_{2}, \overline{\mathcal{M}}_{4}\right]\right]\right) .
$$


11.5. The structure of the polynomials $\pi_{2 N}(\lambda)$. In [J10b], we introduced the polynomials $\pi_{2 N}(\lambda)$ of degree $N-1.14$ These polynomials satisfy systems of respective $N$ factorization relations which allow to describe them in terms of compositions of GJMS-operators. In fact, the following formula should be compared with Theorem 4.1. We have

$$
\pi_{2 N}(\lambda)=\frac{1}{(N-1) !} \sum_{|I|=N} \frac{b_{N}\left(\lambda+\frac{n}{2}-N\right)}{\left(\lambda+\frac{n}{2}-2 N+I_{l}\right)} m_{I} P_{2 I},
$$

where $b_{N}(\lambda)=\prod_{k=0}^{N-1}(\lambda-k)$. In particular, the constant term $\pi_{2 N}(-n / 2+N)$ equals $P_{2 N}$, up to a sign, and the operator $\mathcal{M}_{2 N}$ is a constant multiple of the leading coefficient of $\pi_{2 N}(\lambda)$. Moreover, it turns out that the sub-leading coefficient $\mathcal{M}_{2 N}^{(2)}$ of $\pi_{2 N}(\lambda)$ can be expressed in terms of the leading coefficients according to

$$
\mathcal{M}_{2 N}^{(2)}=-\frac{N}{2} \mathcal{M}_{2 N}-\sum_{k=1}^{N-1}\left(\begin{array}{c}
N-2 \\
N-1-k
\end{array}\right)\left(\begin{array}{c}
N-1 \\
N-1-k
\end{array}\right) \mathcal{M}_{2 k} \mathcal{M}_{2 N-2 k} .
$$

In fact, the identity (11.6) is a consequence of the quadratic relation (6.8) for the coefficients $m_{I}$. Since the operators $\mathcal{M}_{2}, \mathcal{M}_{4}, \cdots$ are of second order, the identity (11.6) implies that the sub-leading coefficients are of fourth order. In other words, the sub-leading coefficient of $\pi_{2 N}(\lambda)$ defines a universal linear combination of order $2 N$ compositions of GJMS-operators which actually is of order four. This cancellation result confirms an expectation formulated in J10b (see the comments after Theorem 4.2). Similar results are expected for all coefficients of the polynomials $\pi_{2 N}(\lambda)$. In the extreme case, the inversion formula (Theorem 2.1) can be regarded as a description of its constant term $P_{2 N}$ along these lines. The full structure of $\pi_{2 N}(\lambda)$ as a function of $\mathcal{M}_{2}, \ldots, \mathcal{M}_{2 N}$ remains to be determined, however.

11.6. $Q_{2 N}^{r e s}(\lambda)$ and the universal recursive formula for $Q_{2 N}$. The representation formula (11.5) is closely related to the following formula for the so-called $Q$-curvature polynomial [J09a], BJ10]

$$
Q_{2 N}^{r e s}(\lambda) \stackrel{\text { def }}{=}-(-1)^{N} D_{2 N}^{r e s}(\lambda)(1)
$$

We have

$$
\begin{aligned}
Q_{2 N}^{r e s}(\lambda)=-\frac{1}{(N-1) !} \lambda\left(\prod_{j=1}^{N-1}\right. & \left(\lambda+\frac{n}{2}-N-j\right)(-1)^{N} Q_{2 N} \\
& \left.+\sum_{|I|+a=N} \frac{b_{N}\left(\lambda+\frac{n}{2}-N\right)}{\left(\lambda+\frac{n}{2}-2 N+I_{l}\right)} m_{(I, a)}(-1)^{a} P_{2 I}\left(Q_{2 a}\right)\right) .
\end{aligned}
$$

Similarly as Theorem 4.1 follows from the full systems of respective $2 N$ factorization identities for residue families of order $2 N$, the formula (11.8) follows from (the consequences for the $Q$-curvature polynomials of) the respective systems of the first $N$ factorization identities for residue families of order $2 N$ (and the vanishing result

\footnotetext{
${ }^{14}$ The operator-valued polynomials $\pi_{2 N}(\lambda)$ are not to be confused with the polynomials defined in (4.1).
} 
$Q_{2 N}^{r e s}(0)=(15)$. Note that the equality of the two expressions for the leading coefficient of $Q_{2 N}^{r e s}(\lambda)$ which follow from the definition (11.5) and from (11.8) is equivalent to the universal recursive formula (1.14) for $Q_{2 N}$. For more details we refer to [J10b].

11.7. Contributions with many derivatives. Theorem 1.1 easily yields a description of the contributions to $P_{2 N}$ with strictly more than $2 N-4$ derivatives. In fact, these are contained in the contributions to (1.8) which are defined by the compositions $I=(1, \ldots, 1)$ and

$$
I_{k}=(\underbrace{1, \ldots, 1}_{k}, 2, \underbrace{1, \ldots, 1}_{N-k-2}), k=0, \ldots, N-2
$$

of size $N$. Now $I=(1, \ldots, 1)$, i.e., $\mathcal{M}_{2}^{N}$ yields the contributions $\Delta^{N}$ and

$$
-\left(\frac{n}{2}-1\right) \sum_{k=0}^{N-1} \Delta^{k}\left(\mathrm{~J} \Delta^{N-k-1}\right)
$$

Moreover, using

$$
n_{I_{k}}=\left(\begin{array}{c}
k+1 \\
1
\end{array}\right)\left(\begin{array}{c}
N-k-1 \\
1
\end{array}\right)=(k+1)(N-k-1)
$$

we obtain the contribution

$$
-\sum_{k=0}^{N-2} 4(k+1)(N-k-1) \Delta^{k} \delta(\mathrm{P} d) \Delta^{N-k-2} .
$$

Thus, by $\delta(\mathrm{P} d)=-(\mathrm{P}, \mathrm{Hess})-(d \mathrm{~J}, d)$, the desired contributions are covered by

$$
\begin{aligned}
\Delta^{N}+4 \sum_{k=0}^{N-2}(k+1)(N-k-1) \Delta^{k}[(\mathrm{P}, \text { Hess })+(d \mathrm{~J}, d)] \Delta^{N-k-2} & \\
& -\left(\frac{n}{2}-1\right) \sum_{k=0}^{N-1} \Delta^{k}\left(\mathrm{~J} \Delta^{N-k-1}\right) .
\end{aligned}
$$

We observe that (11.9) coincides with the sum

$$
\begin{aligned}
\Delta^{N}+2 \sum_{k=0}^{N-2}(k+1)(N-k-1) \Delta^{k}[2(\mathrm{P}, \text { Hess }) & +(d \mathrm{~J}, d)] \Delta^{N-k-2} \\
& +\sum_{k=0}^{N-1}\left(2 k+2-N-\frac{n}{2}\right) \Delta^{k}\left(\mathrm{~J} \Delta^{N-k-1}\right),
\end{aligned}
$$

up to terms with at most $2 N-4$ derivatives 16 In fact, the difference of (11.9) and (11.10) equals

$$
2 \sum_{k=0}^{N-2}(k+1)(N-k-1) \Delta^{k}(d \mathrm{~J}, d) \Delta^{N-k-2}-\sum_{k=0}^{N-1}(2 k+1-N) \Delta^{k}\left(\mathrm{~J} \Delta^{N-k-1}\right) .
$$

\footnotetext{
${ }^{15}$ see Theorem 1.6.6 in BJ10

16 The operator $2(\mathrm{P}$, Hess $)+(d J, d)=-2 \delta(\mathrm{P} d)-(d J, d)$ is the metric variation $\left.2(d / d t)\right|_{t=0}\left(\Delta_{g-t \mathrm{P}}\right)$ of the Laplacian.
} 
Using $2(d \mathrm{~J}, d u)=\Delta(\mathrm{J} u)-\mathrm{J} \Delta(u)-\Delta(\mathrm{J}) u$, we find that (modulo terms with at most $2 N-4$ derivatives) the latter sum equals

$$
\begin{aligned}
\sum_{k=0}^{N-2}(k+1)(N-k-1) \Delta^{k+1}\left(\mathrm{~J} \Delta^{N-k-2}\right)- & \sum_{k=0}^{N-2}(k+1)(N-k-1) \Delta^{k}\left(\mathrm{~J} \Delta^{N-k-1}\right) \\
& -\sum_{k=0}^{N-1}(2 k+1-N) \Delta^{k}\left(\mathrm{~J} \Delta^{N-k-1}\right)=0
\end{aligned}
$$

An alternative direct proof of (11.10) can be found in [M10] (Proposition 2.1). We also observe that (11.9) can be written in the form

$$
\Delta^{N}+\sum_{k=0}^{N-2} \Delta^{k} \delta((n-2) \mathrm{J}-4(k+1)(N-k+1) \mathrm{P}) d \Delta^{N-k-2}+\left(\frac{n}{2}-1\right) \sum_{k=1}^{N-2} \Delta^{k}\left(\mathrm{~J} \Delta^{N-1-k}\right) .
$$

This formula explains the structure of the first four terms in (10.15). In order to prove the assertion, we consider the difference

$$
-\left(\frac{n}{2}-1\right) \sum_{k=0}^{N-1} \Delta^{k}\left(\mathrm{~J} \Delta^{N-k-1}\right)-\sum_{k=0}^{N-2} \Delta^{k} \delta((n-2) \mathrm{J}) d \Delta^{N-k-2}
$$

In view of

$$
\Delta^{N-1}(\mathrm{~J} u)=\Delta^{N-2}(\Delta(\mathrm{J}) u+2(d \mathrm{~J}, d u)+\mathrm{J} \Delta(u))
$$

and $\delta(\mathrm{J} d)=-\mathrm{J} \Delta-(d \mathrm{~J}, d)$, the last two terms of the first sum cancel against the last term of the second sum, up to a term with at most $2 N-4$ derivatives. The remaining differences are given by

$$
-\left(\frac{n}{2}-1\right) \Delta^{k}\left(\mathrm{~J} \Delta^{N-k-1}\right)-(n-2) \Delta^{k} \delta(\mathrm{J} d) \Delta^{N-k-2}=\left(\frac{n}{2}-1\right) \Delta^{k+1}\left(\mathrm{~J} \Delta^{N-k-2}\right)
$$

for $k=0, \ldots, N-3$, up to terms of order $\leq 2 N-4$. This proves the claim.

The direct determination of the above contributions from the ambient metric (as in [M10]) involves the first metric variation of the Laplacian (see footnote 16). The terms with fewer derivatives also contain contributions which come from higher-order metric variations of the Laplacian. From this point of view, the method of residue families can be regarded as a way to avoid dealing with these metric variations.

11.8. The restriction property revisited. In Section 6, we derived the restriction property

$$
\mathcal{M}_{2 N} i^{*}=i^{*} \overline{\mathcal{M}}_{2 N} \quad \text { for } N \geq 2
$$

from the factorization relations of residue families 17 But Theorem 1.1 identifies the generating function $\mathcal{H}(r)$ of the sequence $\mathcal{M}_{2}, \mathcal{M}_{4}, \ldots$ with a natural second-order differential operator. We show that the restriction property directly can be derived from this identification.

\footnotetext{
${ }^{17}$ Of course, the parameter $N$ is also subject to the restriction $2 N \leq n$ for even $n$.
} 
For this purpose, we define a sequence of natural second-order operators $\vartheta_{2 N}(g)$ by the identity

$$
\sum_{N \geq 1} \vartheta_{2 N}(g) \frac{1}{(N-1) !^{2}}\left(\frac{r^{2}}{4}\right)^{N-1}=\mathcal{D}(g)(r),
$$

where $\mathcal{D}(g)(r)$ is the operator on the right-hand side of (1.9). A simple calculation shows that $\mathcal{D}(0)=\vartheta_{2}=P_{2}$.

Proposition 11.1. For $N \geq 2$, the operators $\vartheta_{2 N}$ satisfy the restriction property

$$
i^{*} \bar{\vartheta}_{2 N}=\vartheta_{2 N} i^{*} \text {. }
$$

Here $\vartheta_{2 N}$ and $\bar{\vartheta}_{2 N}$ are defined with respect to the metrics $g$ and $\bar{g}$.

Proof. Theorem 7.2 implies that

$$
g(0, s)=d r^{2}+g(s) .
$$

Hence, by the structure of the principal part of the right-hand side of (11.12), the relation (11.13) is equivalent to the restriction property

$$
i^{*} \bar{\vartheta}_{2 N}(1)=\vartheta_{2 N}(1) \quad \text { for } N \geq 2
$$

of the zeroth-order terms. A second consequence of Eq. (11.14) is that the curvature quantities

$$
v(r, s)=\frac{\operatorname{vol}(g(r, s))}{\operatorname{vol}(g(r, 0))}=\frac{\operatorname{vol}(g(r, s))}{\operatorname{vol}\left(d r^{2}+g(r)\right)} \quad \text { and } \quad v(r)=\frac{\operatorname{vol}(g(r))}{\operatorname{vol}(g)}
$$

satisfy the relation

$$
v(0, s)=v(s)
$$

Hence we also have

$$
w(0, s)=w(s) .
$$

Now the arguments in the proof of Theorem 8.1 given in Section 8.1 (page 52) prove that the operators $\vartheta_{2 N}$ satisfy the recursive relations

$$
\sum_{k=0}^{N-1}\left(2^{k} \frac{(N-1) !}{(N-1-k) !}\right)^{2} \vartheta_{2 N-2 k}\left(w_{2 k}\right)=\left(\frac{n}{2}-N\right)(N-1) ! N ! 2^{2 N} w_{2 N}
$$

(see $(8.25)$ ). We apply these relations to prove (11.15) using induction on $N$. First of all, Lemma 11.1 gives $i^{*} \bar{\vartheta}_{4}(1)=\vartheta_{4}(1)$. Now assume that we have proved

$$
i^{*} \bar{\vartheta}_{2 k}(1)=\vartheta_{2 k}(1) \quad \text { for } k=1, \ldots, N \text {. }
$$

Then, by arguments as above, we also have

$$
i^{*} \bar{\vartheta}_{2 k}=\vartheta_{2 k} i^{*} \quad \text { for } k=1, \ldots, N .
$$

But these properties together with the relation

$$
i^{*} \bar{P}_{2}\left(\bar{w}_{2 N}\right)-P_{2}\left(w_{2 N}\right)=(2 N+2) w_{2 N+2}
$$

(see Lemma 11.2) imply

$$
i^{*} \bar{\vartheta}_{2 N+2}(1)=\vartheta_{2 N+2}(1) .
$$


In fact, by taking the difference of (11.17) for $g$ and $\bar{g}$ (and $N$ replaced by $N+1$ ) and using $i^{*} \bar{w}_{2 N+2}=w_{2 N+2}($ see (11.16) $)$ and (11.18), we obtain

$$
i^{*} \bar{\vartheta}_{2 N+2}(1)-\vartheta_{2 N+2}(1)+\left(2^{N} N !\right)^{2}\left(i^{*} \bar{P}_{2}\left(w_{2 N}\right)-P_{2}\left(w_{2 N}\right)\right)=N !(N+1) ! 2^{2 N+1} w_{2 N+2} .
$$

Now (11.19) shows that

$$
i^{*} \bar{\vartheta}_{2 N+2}(1)=\vartheta_{2 N+2}(1) .
$$

This completes the induction.

In order to complete the proof of Proposition 11.1, it remains to prove the following two results.

Lemma 11.1. $i^{*} \bar{\vartheta}_{4}=\vartheta_{4}$

Proof. A direct calculation of the coefficient of $r^{2}$ on the right-hand side of (11.12) shows that

$$
\vartheta_{4}=(n-4)|\mathrm{P}|^{2}+\mathrm{J}^{2}-\Delta \mathrm{J}
$$

(see (10.12) ). Thus, the assertion is equivalent to

$$
(n-3) i^{*}|\overline{\mathrm{P}}|^{2}+i^{*} \overline{\mathrm{J}}^{2}-i^{*} \bar{\Delta} \overline{\mathrm{J}}=(n-4)|\mathrm{P}|^{2}+\mathrm{J}^{2}-\Delta \mathrm{J} .
$$

But

$$
i^{*} \overline{\mathrm{J}}=\mathrm{J},\left.\quad \overline{\mathrm{P}}\right|_{r=0}=\left(\begin{array}{cc}
0 & 0 \\
0 & \mathrm{P}
\end{array}\right) \quad \text { and } \quad i^{*}\left(\partial^{2} / \partial r^{2}\right)(\overline{\mathrm{J}})=|\mathrm{P}|^{2}
$$

(for detailed proofs see [J09a, Section 6.11) imply that

$$
i^{*}|\overline{\mathrm{P}}|^{2}=|\mathrm{P}|^{2} \quad \text { and } \quad i^{*} \bar{\Delta} \overline{\mathrm{J}}-\Delta \mathrm{J}=|\mathrm{P}|^{2} .
$$

This proves (11.20).

Lemma 11.2. For $N \geq 1$, we have

$$
i^{*} \bar{P}_{2}\left(\bar{w}_{2 N-2}\right)-P_{2}\left(w_{2 N-2}\right)=2 N w_{2 N},
$$

where $P_{2}$ and $\bar{P}_{2}$ denote the respective Yamabe-operators of $g$ and $\bar{g}$.

Proof. We reformulate the identities (11.21) in the form

$$
i^{*} \bar{P}_{2}(w(r, s))-P_{2}(w(s))=s^{-1} \partial w(s) / \partial s .
$$

Now an easy calculation using $i^{*} \bar{J}=\mathrm{J}$ and $w(0, s)=w(s)$ (see (11.16) ) shows that this identity is equivalent to (8.38). The proof is complete.

11.9. $\mathcal{M}_{2 N}$ and $\overline{\mathcal{M}}_{2 N}$ for round spheres. We determine explicit summation formulas for the operators $\mathcal{M}_{2 N}$ and $\overline{\mathcal{M}}_{2 N}$ for round spheres $\mathbb{S}^{n}$. First, we recall the summation formula ([J09b], Theorem 6.1)

$$
\mathcal{M}_{2 N}=N !(N-1) ! P_{2}, \quad N \geq 1 \text {. }
$$

Next, we have

$$
\bar{g}=d r^{2}+\left(1-r^{2} / 4\right)^{2} g_{\mathbb{S}^{n}}
$$

and Theorem 1.1 implies the following result. 
Proposition 11.2. We have

$$
\overline{\mathcal{M}}_{2 N}=(N-1) ! N !\left(1-r^{2} / 4\right)^{-N-1} P_{2}\left(\mathbb{S}^{n}\right)
$$

for $N \geq 2$.

Proof. Theorem 7.2 gives

$$
g(r, s)=d r^{2}+\left(1-\left(r^{2}+s^{2}\right) / 4\right)^{2} g_{\mathbb{S}^{n}} .
$$

Now Theorem 1.1 and the identity

$$
\left(1-\frac{r^{2}+s^{2}}{4}\right)^{-2}=\sum_{N \geq 1} N\left(1-\frac{r^{2}}{4}\right)^{-N-1}\left(\frac{s^{2}}{4}\right)^{N-1}
$$

show that for $N \geq 2$ the principal part of $\overline{\mathcal{M}}_{2 N}$ equals

$$
(N-1) ! N !\left(1-r^{2} / 4\right)^{-N-1} \Delta_{\mathbb{S}^{n}}
$$

Moreover, using

$$
w(r, s)=\left(1-\left(r^{2}+s^{2}\right) / 4\right)^{\frac{n}{2}}\left(1-r^{2} / 4\right)^{-\frac{n}{2}}
$$

and

$$
-\delta_{\bar{g}}\left(g(r, s)^{-1} d\right)=\frac{\partial^{2}}{\partial r^{2}}-\frac{n}{2} r\left(1-\frac{r^{2}}{4}\right)^{-1} \frac{\partial}{\partial r}+\left(1-\frac{r^{2}+s^{2}}{4}\right)^{-2} \Delta_{\mathbb{S}^{n}}
$$

a straightforward calculation shows that the potential of the second-order operator on the right-hand side of (1.9) equals

$$
-\left(1-\frac{r^{2}+s^{2}}{4}\right)^{-2} \frac{n}{2}\left(\frac{n}{2}-1\right)+\left(1-\frac{r^{2}}{4}\right)^{-2} \frac{n}{2}\left(\frac{n-1}{2} \frac{r^{2}}{4}-\frac{1}{2}\right) .
$$

Now Eq. (11.23) and Eq. (11.24) prove the assertion for the zeroth-order terms in (11.22).

Note that the potential (11.24) restricts to

$$
-\frac{n}{2} \frac{n-1}{2}\left(1-\frac{r^{2}}{4}\right)^{-1}=-\frac{n-1}{2} \bar{\jmath}
$$

for $s=0$. Here we use that

$$
\overline{\mathrm{J}}=\mathrm{J}(\bar{g})=-\frac{1}{2 r} \operatorname{tr}\left(\frac{\dot{g}_{r}}{g_{r}}\right)=\frac{n}{2}\left(1-\frac{r^{2}}{4}\right)^{-1} .
$$

Note also that the formula (11.22) confirms the restriction property and the commutator relations in that special case.

\section{Appendix}

In this appendix, we collect a number of important formulas and give the details of some central calculations. In particular, we give direct proofs of some low-order special cases of the symmetry relation $g_{(2 a, 2 b)}=g_{(2 b, 2 a)}$ (see Eq. (7.6)), and of the relation between $g_{(4,4)}$ and $g_{(8)}$. Moreover, we extract from these considerations an improved version of an algorithm of [G09]. 
12.1. The operators $\mathcal{M}_{2 N}$ for $N \leq 5$. In the present section, we display the explicit definitions of the low-order cases $N \leq 5$ of the operators $\mathcal{M}_{2 N}$. These operators are given by the formulas

$$
\begin{gathered}
\mathcal{M}_{2}=P_{2}, \\
\mathcal{M}_{4}=P_{4}-P_{2}^{2}, \\
\mathcal{M}_{6}=P_{6}-\left(2 P_{2} P_{4}+2 P_{4} P_{2}\right)+3 P_{2}^{3}, \\
\mathcal{M}_{8}=P_{8}-\left(3 P_{2} P_{6}+3 P_{6} P_{2}+9 P_{4}^{2}\right)+\left(12 P_{2}^{2} P_{4}+12 P_{4} P_{2}^{2}+8 P_{2} P_{4} P_{2}\right)-18 P_{2}^{4}
\end{gathered}
$$

and

$$
\begin{aligned}
\mathcal{M}_{10}= & P_{10}-\left(4 P_{2} P_{8}+4 P_{8} P_{2}+24 P_{4} P_{6}+24 P_{6} P_{4}\right) \\
& +\left(60 P_{2} P_{4}^{2}+60 P_{4}^{2} P_{2}+30 P_{2}^{2} P_{6}+30 P_{6} P_{2}^{2}+15 P_{2} P_{6} P_{2}+80 P_{4} P_{2} P_{4}\right) \\
& -\left(120 P_{2}^{3} P_{4}+120 P_{4} P_{2}^{3}+80 P_{2}^{2} P_{4} P_{2}+80 P_{2} P_{4} P_{2}^{2}\right)+180 P_{2}^{5} .
\end{aligned}
$$

The sums in (12.3), (12.4) and (12.5) involve 4, 8 and 16 terms, respectively. In these formulas, the parentheses contain the respective contributions with the same number of factors.

12.2. The restriction property of $g_{(4)}$. Here we prove that

$$
g_{(4)}=\frac{1}{4}\left(\mathrm{P}^{2}-\frac{\mathcal{B}}{n-4}\right)
$$

satisfies the restriction property

$$
\left.\bar{g}_{(4)}\right|_{r=0}=g_{4} .
$$

In other words, we prove the symmetry relation $g_{(4,0)}=g_{(0,4)}$. First of all,

$$
\left.\overline{\mathrm{P}}\right|_{r=0}=\mathrm{P},
$$

i.e., $g_{(2,0)}=g_{(0,2)}$ (see Lemma 12.1) shows that the relation (12.6) is equivalent to the restriction property

$$
\left.(n-4) \mathcal{B}\left(d r^{2}+g_{r}\right)\right|_{r=0}=(n-3) \mathcal{B}(g)
$$

of the Bach tensor. In order to prove (12.7), we use the transformation rule

$$
e^{2 \varphi} \hat{\mathcal{B}}_{i j}=\mathcal{B}_{i j}-(n-4)\left(C_{i k j}+C_{j k i}\right) \varphi^{k}+(n-4) W_{i r s j} \varphi^{r} \varphi^{s},
$$

where $C$ and $W$ denote the Cotton and Weyl tensor, respectively 18 Since $r \partial / \partial r$ is dual to $d \log r=d r / r$, (12.8) implies that

$$
\begin{aligned}
r^{2} \mathcal{B}\left(d r^{2}+g_{r}\right)(X, Y)=\mathcal{B}\left(g_{+}\right)(X, Y) & \\
-(n-3)\left(C\left(g_{+}\right)(X, r \partial / \partial r, Y)\right. & \left.+C\left(g_{+}\right)(Y, r \partial / \partial r, X)\right) \\
& +(n-3) W\left(g_{+}\right)(X, r \partial / \partial r, r \partial / \partial r, Y) .
\end{aligned}
$$

But the Bach tensor and the Cotton tensor of the Einstein metric $g_{+}$vanish. Thus, we find

$$
\mathcal{B}\left(d r^{2}+g_{r}\right)(X, Y)=(n-3) W\left(g_{+}\right)(X, \partial / \partial r, \partial / \partial r, Y) .
$$

\footnotetext{
${ }^{18}$ Here we use the conventions of [J09a]. In particular, the curvature tensor $R$ and the Weyl tensor $W$ have opposite signs as in [FG07. Moreover, we define the Cotton tensor by $C(X, Y, Z)=$ $\nabla_{X}(\mathrm{P})(Y, Z)-\nabla_{Y}(\mathrm{P})(X, Z)$.
} 
Hence the assertion

$$
i^{*} \mathcal{B}\left(d r^{2}+g_{r}\right)=(n-3) /(n-4) \mathcal{B}(g)
$$

follows from the fact that (on $r=0$ ) the Weyl-curvature term yields $\mathcal{B}(g) /(n-4)$. In order to prove this, we apply the decomposition $R=W-\mathrm{P} \otimes g$ for $g_{+}$. Using $\mathrm{P}\left(g_{+}\right)=-\frac{1}{2} g_{+}$, we find

$$
W\left(g_{+}\right)(X, \partial / \partial r, \partial / \partial r, Y)=R\left(g_{+}\right)(X, \partial / \partial r, \partial / \partial r, Y)+\frac{1}{r^{2}} g_{+}(X, Y)
$$

for $X, Y \in \mathfrak{X}(M)$. Now a routine calculation [F06] shows that

$$
R\left(g_{+}\right)(\cdot, \partial / \partial r, \partial / \partial r, \cdot)=-\frac{1}{r^{2}} g_{+}(\cdot, \cdot)+\left.\tilde{R}(\cdot, \partial / \partial \rho, \partial / \partial \rho, \cdot)\right|_{t=1, \rho=-r^{2} / 2}
$$

Here $\tilde{R}$ is the curvature of the ambient metric

$$
\tilde{g}=2 t d t d \rho+2 \rho d t^{2}+t^{2} g(\rho), \quad g(0)=g
$$

of $g$, and the dots indicate vector fields in $\mathfrak{X}(M)$. Hence

$$
W\left(g_{+}\right)(\cdot, \partial / \partial r, \partial / \partial r, \cdot)=\left.\tilde{R}(\cdot, \partial / \partial \rho, \partial / \partial \rho, \cdot)\right|_{t=1, \rho=-r^{2} / 2},
$$

i.e.,

$$
-\bar{\Omega}^{(1)}(X, Y)=\left.\tilde{R}(X, \partial / \partial \rho, \partial / \partial \rho, Y)\right|_{t=1, \rho=-r^{2} / 2}, X, Y \in \mathfrak{X}(M),
$$

where $\Omega^{(1)}=-\mathcal{B} /(n-4)$ is the first extended obstruction tensor [G09]. Therefore, the identity

$$
\left.\tilde{R}(\cdot, \partial / \partial \rho, \partial / \partial \rho, \cdot)\right|_{t=1, \rho=0}=\mathcal{B} /(n-4)=-\Omega^{(1)}
$$

(see [FG07]) proves the assertion. Finally,

$$
\mathcal{B}\left(d r^{2}+g_{r}\right)(X, \partial / \partial r)=(n-3) W\left(g_{+}\right)(X, \partial / \partial r, \partial / \partial r, \partial / \partial r)=0
$$

shows that the restriction of $\mathcal{B}\left(d r^{2}+g_{r}\right)$ to $r=0$ has no non-trivial normal components. This completes the proof of (12.7).

12.3. The symmetry relation $g_{(4,2)}=g_{(2,4)}$. In the present section, we prove the symmetry relation

We consider the tensor

$$
g_{(4,2)}=g_{(2,4)}
$$

$$
\frac{1}{4}\left(\overline{\mathrm{P}}^{2}-\frac{\overline{\mathcal{B}}}{n-3}\right)
$$

as a function of $r$. By the above results, the constant term of its Taylor series is

$$
\frac{1}{4}\left(\mathrm{P}^{2}-\frac{\mathcal{B}}{n-4}\right)
$$

We determine its Taylor coefficient $g_{(2,4)}$ of $r^{2}$. Using

$$
\overline{\mathrm{P}}=-\frac{1}{2 r} \dot{g}_{r}=\mathrm{P}-\frac{r^{2}}{2}\left(\mathrm{P}^{2}-\frac{\mathcal{B}}{n-4}\right)+\cdots
$$

(see Lemma 12.1), we find that $\overline{\mathrm{P}}^{2}$ is given by

$$
\overline{\mathrm{P}}_{i r} \bar{g}^{r k} \overline{\mathrm{P}}_{k j}=\left(\mathrm{P}_{i r}-\frac{r^{2}}{2}\left(\mathrm{P}_{i r}^{2}-\frac{\mathcal{B}_{i r}}{n-4}\right)+\cdots\right)\left(g^{r k}+r^{2} \mathrm{P}^{r k}+\cdots\right)
$$




$$
\times\left(\mathrm{P}_{k j}-\frac{r^{2}}{2}\left(\mathrm{P}_{k j}^{2}-\frac{\mathcal{B}_{k j}}{n-4}\right)+\cdots\right) .
$$

The Taylor coefficient of $r^{2}$ of the latter product is the sum of

$$
\mathrm{P}_{i r} \mathrm{P}^{r k} \mathrm{P}_{k j}=\left(\mathrm{P}^{3}\right)_{i j}
$$

and

$$
-\left(\mathrm{P}^{3}\right)_{i j}+\frac{1}{2(n-4)}\left(\mathcal{B}_{i r} \mathrm{P}_{j}^{r}+\mathrm{P}_{i}^{r} \mathcal{B}_{r j}\right) .
$$

Hence, in terms of $\Omega^{(1)}=-\mathcal{B} /(n-4)$, the Taylor coefficient of $r^{2}$ of $\overline{\mathrm{P}}^{2} / 4$ is given by

$$
-\frac{1}{8}\left(\mathrm{P}_{i}^{r} \Omega_{j r}^{(1)}+\mathrm{P}_{j}^{r} \Omega_{i r}^{(1)}\right) \text {. }
$$

Next, we use the relation

$$
\frac{\overline{\mathcal{B}}}{n-3}=\left.\tilde{R}\left(\cdot, e_{\infty}, e_{\infty}, \cdot\right)\right|_{t=1, \rho=-r^{2} / 2}=\left.\tilde{R}_{\cdot \infty \infty} \cdot\right|_{t=1, \rho=-r^{2} / 2}, \quad e_{\infty}=\partial / \partial \rho
$$

to determine the Taylor coefficient of $r^{2}$ of $-\overline{\mathcal{B}} /(4(n-3))$. Now

$$
\left.\tilde{R}_{i \infty \infty j}\right|_{t=1, \rho=-r^{2} / 2}=\left.\tilde{R}_{i \infty \infty j}\right|_{t=1, \rho=0}-\partial \tilde{R}_{i \infty \infty j} /\left.\partial \rho\right|_{t=1, \rho=0} \frac{r^{2}}{2}+\cdots .
$$

But $\tilde{\nabla}_{\rho}(\partial / \partial \rho)=0$ implies

$$
\begin{aligned}
\partial \tilde{R}_{i \infty \infty j} / \partial \rho=\tilde{\nabla}_{\rho}(\tilde{R})\left(e_{i}, e_{\infty}, e_{\infty}, e_{j}\right) & \\
& +\tilde{R}\left(\tilde{\nabla}_{\rho}\left(e_{i}\right), e_{\infty}, e_{\infty}, e_{j}\right)+\tilde{R}\left(e_{i}, e_{\infty}, e_{\infty}, \tilde{\nabla}_{\rho}\left(e_{j}\right)\right) .
\end{aligned}
$$

A calculation of Christoffel symbols ([FG07], Eq. (3.16) or [F06]) shows that

$$
\left.\tilde{\nabla}_{\rho}\left(e_{i}\right)\right|_{t=1, \rho=0}=\mathrm{P}_{i}^{k} e_{k} .
$$

Hence

$$
\partial \tilde{R}_{i \infty \infty j} /\left.\partial \rho\right|_{t=1, \rho=0}=-\Omega_{i j}^{(2)}-\mathrm{P}_{i}^{k} \Omega_{k j}^{(1)}-\mathrm{P}_{j}^{k} \Omega_{i k}^{(1)} .
$$

(see Eq. (12.20) $)$. Therefore, the Taylor coefficient of $r^{2}$ of $-\overline{\mathcal{B}}_{i j} /(4(n-3))$ is

$$
-\frac{1}{8}\left(\Omega_{i j}^{(2)}+\mathrm{P}_{i}^{k} \Omega_{k j}^{(1)}+\mathrm{P}_{j}^{k} \Omega_{i k}^{(1)}\right) .
$$

Summarizing (12.13) and (12.16), we find the coefficient

$$
-\frac{1}{8}\left(\Omega_{i j}^{(2)}+2 \mathrm{P}_{i}^{k} \Omega_{k j}^{(1)}+2 \mathrm{P}_{j}^{k} \Omega_{i k}^{(1)}\right) .
$$

But the quantity (12.17) coincides with the coefficient $g_{(4,2)}$ of $r^{4}$ in the Taylor expansion of $-\overline{\mathrm{P}}$. In fact, the latter assertion follows by combining the formula (see [G09], Eq. $(2.22))$

$$
g_{r}=g-\mathrm{P} r^{2}+\left(\mathrm{P}^{2}+\Omega^{(1)}\right) \frac{r^{4}}{4}-\left(\Omega^{(2)}+2\left(\mathrm{P} \Omega^{(1)}+\Omega^{(1)} \mathrm{P}\right)\right) \frac{r^{6}}{24}+\cdots
$$

with Lemma 12.1. This proves the symmetry (12.10). 
12.4. The coefficient $g_{(4,4)}$. Theorem 7.2 implies that

$$
g_{(4,4)}=\left(\begin{array}{l}
4 \\
2
\end{array}\right) g_{(8)} \text {. }
$$

Here we present a direct proof of this relation. The following arguments also serve as an illustration of an algorithm described in Section 12.5.

In order to make the left-hand side of (12.18) explicit, we have to determine the coefficient of $r^{4}$ of

$$
\overline{\mathrm{P}}^{2}+\bar{\Omega}^{(1)}
$$

First, using

$$
\overline{\mathrm{P}}=\mathrm{P}-\frac{r^{2}}{2}\left(\mathrm{P}^{2}+\Omega^{(1)}\right)+\frac{r^{4}}{8}\left(\Omega^{(2)}+2\left(\mathrm{P} \Omega^{(1)}+\Omega^{(1)} \mathrm{P}\right)\right)+\cdots
$$

(Lemma 12.1), we find that $\overline{\mathrm{P}}^{2}$ yields the contribution

$$
r^{4}\left(\frac{1}{8}\left(\Omega^{(2)} \mathrm{P}+\mathrm{P} \Omega^{(2)}\right)+\frac{1}{4} \mathrm{P} \Omega^{(1)} \mathrm{P}+\frac{1}{4} \Omega^{(1)} \Omega^{(1)}\right) .
$$

Next, using

$$
\bar{\Omega}_{i j}^{(1)}=\Omega_{i j}^{(1)}\left(d r^{2}+g_{r}\right)=-\left.\tilde{R}_{i \infty \infty j}\right|_{t=1, \rho=-r^{2} / 2}
$$

(see Eq. (12.9)), we find that the second term in (12.19) yields the contribution

$$
-\left.\frac{1}{8} r^{4}(\partial / \partial \rho)^{2}\left(\tilde{R}_{i \infty \infty j}\right)\right|_{t=1, \rho=0}
$$

But

$$
\begin{aligned}
(\partial / \partial \rho)^{2}\left(\tilde{R}_{i \infty \infty j}\right) & =\tilde{\nabla}_{\rho}^{2}(\tilde{R})_{i \infty \infty j} \\
& +2 \tilde{\nabla}_{\rho}(\tilde{R})\left(\tilde{\nabla}_{\rho}\left(\partial_{i}\right), \partial_{\infty}, \partial_{\infty}, \partial_{j}\right)+2 \tilde{\nabla}_{\rho}(\tilde{R})\left(\partial_{i}, \partial_{\infty}, \partial_{\infty}, \tilde{\nabla}_{\rho}\left(\partial_{j}\right)\right) \\
& +\tilde{R}\left(\tilde{\nabla}_{\rho}^{2}\left(\partial_{i}\right), \partial_{\infty}, \partial_{\infty}, \partial_{j}\right)+\tilde{R}\left(\partial_{i}, \partial_{\infty}, \partial_{\infty}, \tilde{\nabla}_{\rho}^{2}\left(\partial_{j}\right)\right) \\
& +2 \tilde{R}\left(\tilde{\nabla}_{\rho}\left(\partial_{i}\right), \partial_{\infty}, \partial_{\infty}, \tilde{\nabla}_{\rho}\left(\partial_{j}\right)\right) .
\end{aligned}
$$

By definition (see Eq. (12.20) $)$, we have

$$
-\left.\tilde{\nabla}_{\rho}(\tilde{R})_{i \infty \infty j}\right|_{t=1, \rho=0}=\Omega_{i j}^{(2)} \text { and }-\left.\tilde{\nabla}_{\rho}^{2}(\tilde{R})_{i \infty \infty j}\right|_{t=1, \rho=0}=\Omega_{i j}^{(3)}
$$

recall that our sign convention for $\tilde{R}$ is opposite to that of [G09]. Moreover, using

$$
\tilde{\nabla}_{\rho}\left(\partial_{i}\right)=\frac{1}{2} g^{k r} \dot{g}_{i r} \partial_{k}, \quad g=g(\rho)
$$

([FG07], Eq. (3.16) or [F06]), we obtain the relations

$$
\left.\tilde{\nabla}_{\rho}\left(\partial_{i}\right)\right|_{t=1, \rho=0}=\mathrm{P}_{i}^{k} \partial_{k}
$$

(see Eq. (12.15) ) and

In particular,

$$
\left.\tilde{\nabla}_{\rho}^{2}\left(\partial_{i}\right)\right|_{t=1, \rho=0}=\left(\Omega^{(1)}\right)_{i}^{k} \partial_{k}
$$

$$
\left.\left(\tilde{R}\left(\tilde{\nabla}_{\rho}^{2}\left(\partial_{i}\right), \partial_{\infty}, \partial_{\infty}, \partial_{j}\right)+\tilde{R}\left(\partial_{i}, \partial_{\infty}, \partial_{\infty}, \tilde{\nabla}_{\rho}^{2}\left(\partial_{j}\right)\right)\right)\right|_{t=1, \rho=0}=-2\left(\Omega^{(1)} \Omega^{(1)}\right)_{i j}
$$


These results show that the coefficient of $r^{4}$ of $\bar{\Omega}^{(1)}$ is given by the sum

$$
\frac{1}{8} \Omega^{(3)}+\frac{1}{4}\left(\Omega^{(2)} \mathrm{P}+\mathrm{P} \Omega^{(2)}\right)+\frac{1}{4} \Omega^{(1)} \Omega^{(1)}+\frac{1}{4} \mathrm{P} \Omega^{(1)} \mathrm{P} .
$$

Summarizing the contributions of $\overline{\mathrm{P}}^{2}$ and $\bar{\Omega}^{(1)}$, we obtain the formula

$$
g_{(4,4)}=\frac{1}{32}\left(\Omega^{(3)}+3\left(\Omega^{(2)} \mathrm{P}+\mathrm{P} \Omega^{(2)}\right)+4 \Omega^{(1)} \Omega^{(1)}+4 \mathrm{P} \Omega^{(1)} \mathrm{P}\right) .
$$

But, according to G09], Eq. (2.22), we have

$$
g_{(8)}=\frac{1}{4 ! 2^{3}}\left(\Omega^{(3)}+3\left(\Omega^{(2)} \mathrm{P}+\mathrm{P} \Omega^{(2)}\right)+4 \Omega^{(1)} \Omega^{(1)}+4 \mathrm{P} \Omega^{(1)} \mathrm{P}\right) .
$$

This proves the relation (12.18).

12.5. Some further consequences of Theorem 7.2. First, we prove a formula for the Schouten tensor of $\bar{g}=d r^{2}+g_{r}$. This result (with an alternative proof) can be found also in [J09a], Lemma 6.11.2.

\section{Lemma 12.1.}

$$
\overline{\mathrm{P}}=-\frac{1}{2 r}(\partial / \partial r)\left(g_{r}\right)
$$

In particular,

$$
\left.\overline{\mathrm{P}}\right|_{r=0}=\mathrm{P} .
$$

Proof. We use the fact that $-\overline{\mathrm{P}}$ is the coefficient of $s^{2}$ in the expansion of $g_{++}$. Now, Theorem 7.2 implies that $-\overline{\mathrm{P}}$ has the expansion

$$
\sum_{N \geq 1} N r^{2 N-2} g_{(2 N)}
$$

But the latter sum equals

$$
\frac{1}{2 r} \partial\left(\sum_{N \geq 0} r^{2 N} g_{(2 N)}\right) / \partial r .
$$

This proves the first assertion. The second assertion follows by using $g_{(2)}=-\mathrm{P}$.

Next, we recall that by [G09] the extended obstruction tensors 19

$$
\Omega_{i j}^{(k)}=-\left.\tilde{\nabla}_{\infty}^{(k-1)}(\tilde{R})_{\infty i j \infty}\right|_{t=1, \rho=0}, k \geq 1
$$

together with the Schouten tensor $\mathrm{P}$, are the building blocks of $g_{+}$in the sense that all Taylor coefficients $g_{(2 k)}$ are given by universal expressions involving $\mathrm{P}$ and extended obstruction tensors.

Lemma 12.2. All extended obstruction tensors $\Omega^{(k)}$ have the restriction property

$$
\left.\bar{\Omega}^{(k)}\right|_{r=0}=\Omega^{(k)} .
$$

In particular, $\left.\bar{\Omega}^{(k)}\right|_{r=0}(\cdot, \partial / \partial r)=0$.

\footnotetext{
${ }^{19}$ We recall that the sign conventions for the curvature tensor differ from those in G09. By the additional sign in (12.20), the extended obstruction tensors coincide, however.
} 
Proof. Let $\bar{g}(r)=d r^{2}+g(r)$. By Lemma 7.1, the Taylor series

$$
\left.E(\bar{g}(r))(s)\right|_{r=0}=\bar{g}(0)+\left.s^{2} \bar{g}_{(2)}\right|_{r=0}+\left.s^{4} \bar{g}_{(4)}\right|_{r=0}+\cdots
$$

coincides with

$$
\left(d r^{2}+g\right)+s^{2} g_{(2)}+s^{4} g_{(4)}+\cdots .
$$

Hence we have the restriction relation

$$
\left.\bar{g}_{(2 N)}\right|_{r=0}=g_{(2 N)} .
$$

But, for any metric $g$, the coefficient $g_{(2 N)}$ can be written as a universal expression in terms of the Schouten tensor $\mathrm{P}$ and the extended obstruction tensors $\Omega^{(1)}, \ldots, \Omega^{(N-1)}$ (see [G09], Eq. (2.22)). Thus, the assertion follows from (12.21) by induction.

Finally, we sketch an algorithm which generates formulas for the Taylor coefficients $g_{(2 k)}$ in terms of $\mathrm{P}$ and extended obstruction tensors. This algorithm can be considered as a refined version of the algorithm in the proof of Theorem 1.2 in [G09]. On the one hand, Theorem 7.2 implies

$$
\frac{1}{4}\left(\overline{\mathrm{P}}^{2}+\bar{\Omega}^{(1)}\right)=\sum_{N \geq 2}\left(\begin{array}{c}
N \\
2
\end{array}\right) r^{2 N-4} g_{(2 N)} .
$$

On the other hand, Lemma 12.1 and the relation

$$
\bar{\Omega}^{(1)}(\cdot, \cdot)=-\left.\tilde{R}\left(\cdot, e_{\infty}, e_{\infty}, \cdot\right)\right|_{t=1, \rho=-r^{2} / 2}
$$

on tangential vectors of $M$ (see Eq. (12.9) $)$ can be used to express the $(2 k)^{\text {th }}$ Taylor coefficient of the left-hand side in terms of $\Omega^{(k+1)}$, lower-order extended obstruction tensors and P. This yields the desired formulas for the coefficients $g_{(2 N)}$. For $g_{(8)}$, the details of this algorithm are given in Section 12.4.

\section{REFERENCES}

[AG11] E. Aubry and C. Guillarmou, Conformal harmonic forms, Branson-Gover operators and Dirichlet problem at infinity, Journal Eur. Math. Soc. 13 (2011), 911-957. arXiv:0808.0552

[BJ10] H. Baum and A. Juhl, Conformal Differential Geometry: Q-curvature and Conformal Holonomy. Oberwolfach Seminars 40, 2010.

[B85] T. P. Branson, Differential operators canonically associated to a conformal structure, Math. Scand. 57 (1985), 293-345.

[B95] T. P. Branson, Sharp inequalities, the functional determinant, and the complementary series, Trans. AMS 347 (1995), 3671-3742.

[B05] T. P. Branson, Q-curvature and spectral invariants. Rend. Circ. Mat. Palermo (2) Suppl. 75 (2005), 11-55.

[BG08] T. P. Branson and A. R. Gover. Origins, applications and generalisations of the $Q$ curvature. Acta Appl. Math. 102 no. 2-3 (2008), 131-146.

[CGS08] A. Čap, A. R. Gover and V. Souček, Conformally invariant operators via curved Casimirs: examples. Pure Appl. Math. Q. 6 no 3 (2010), 693-714. arXiv:0808.1978

[DGH08] Z. Djadli, C. Guillarmou and M. Herzlich, Opérateurs géométriques, invariants conformes et variétés asymptotiquement hyperboliques, Panoramas et Synthèses, 26, Société Mathématique de France, 2008.

[FJ] C. Falk and A. Juhl, Universal recursive formulae for Q-curvature. Journal für die reine und angewandte Mathematik, 652 (2011), 113-163. arXiv:0804.2745v2 
[FG85] C. Feffermann and C. R. Graham, Conformal invariants, The mathematical heritage of Élie Cartan (Lyon, 1984), Astérisque, (1985), Numero Hors Serie, 95-116.

[FG07] C. Fefferman and C. R. Graham, The ambient metric. Annals of Math. Studies 178, Princeton University Press, 2012. arXiv:0710.0919v2

[F06] M. Fischmann, Die Ambient-Metrik Konstruktion, Diplomarbeit, Humboldt-Universität, Berlin, 2006.

[Go06] A. R. Gover, Laplacian operators and $Q$-curvature on conformally Einstein manifolds, Math. Ann. 336 no 2 (2006), 311-334. arXiv:math/0506037v3

[Go10] A. R. Gover, Almost Einstein and Poincaré-Einstein manifolds in Riemannian signature, Journal of Geometry and Physics 60 no. 2 (2010), 182-204. arXiv:0803.3510

[GoH04] A. R. Gover and K. Hirachi, Conformally invariant powers of the Laplacian-a complete nonexistence theorem, J. Amer. Math. Soc. 17 no. 2 (2004), 389-405. arXiv:math/0304082v2

[GoP03] A. R. Gover and L. Peterson, Conformally invariant powers of the Laplacian, Qcurvature, and tractor calculus. Comm. Math. Phys. 235 no. 2 (2003), 339-378. arXiv:math-ph/0201030v3

[GJMS92] C. R. Graham, R. Jenne, L. J. Mason and G. A. J. Sparling, Conformally invariant powers of the Laplacian. I. Existence. J. London Math. Soc. 46 no. 2 (1992), 557-565.

[G92] C. R. Graham, Conformally invariant powers of the Laplacian. II. Nonexistence, J. London Math. Soc. 46 no. 2 (1992), 566-576.

[G00] C. R. Graham, Volume and area renormalizations for conformally compact Einstein metrics, Rend. Circ. Mat. Palermo (2) Suppl. 63 (2000), 31-42. arXiv:math/9909042

[G07] C. R. Graham, Conformal powers of the Laplacian via stereographic projection, SIGMA Symmetry Integrability Geom. Methods Appl. 3 (2007), Paper 121, 4p. arXiv:0711.4798v2

[G09] C. R. Graham, Extended obstruction tensors and renormalized volume coefficients, Adv. Math. 220 no. 6 (2009), 1956-1985. arXiv:0810.4203

[GH05] C. R. Graham and K. Hirachi, The ambient obstruction tensor and $Q$-curvature, AdS/CFT correspondence: Einstein metrics and their conformal boundaries, IRMA Lect. Math. Theor. Phys., 8, (2005), 59-71. arXiv:math/0405068

[GJ07] C. R. Graham and A. Juhl, Holographic formula for $Q$-curvature, Adv. Math. 216 no. 2 (2007), 841-853. arXiv:0704.1673

[GW11] C. R. Graham and T. Willse, Parallel tractor extension and ambient metrics of holonomy split $G_{2}$. arXiv:1109.3504

[GZ03] C. R. Graham and M. Zworski, Scattering matrix in conformal geometry, Invent. Math. 152 no. 1 (2003), 89-118. arXiv: math/0109089

[GKP] R. L. Graham, and D. E. Knuth and O. Patashnik, Concrete mathematics. A foundation for computer science, Addison-Wesley Publishing Company Advanced Book Program, (1989),

[J09a] A. Juhl, Families of Conformally Covariant Differential Operators, Q-Curvature and Holography, volume 275 of Progress in Mathematics. Birkhäuser Verlag, 2009.

[J09b] A. Juhl, On conformally covariant powers of the Laplacian. arXiv:0905.3992v3

[J09c] A. Juhl, On Branson's Q-curvature of order eight. Conformal Geometry and Dynamics 15 (2011), 20-43. arXiv:0912.2217

[J10a] A. Juhl, Holographic formula for Q-curvature. II. Adv. in Math. 226 no.4 (2011), 34093425. arXiv: 1003.3989

[J10b] A. Juhl, On the recursive structure of Branson's $Q$-curvature. arXiv:1004.1784.v2

[JK09] A. Juhl and C. Krattenthaler, Summation formulas for GJMS-operators and $Q$-curvatures on the Möbius sphere. arXiv:0910.4840

[K10] C. Krattenthaler, private communication (23.11.2010).

[LN09] T. Leistner and P. Nurowski, Ambient metrics for $n$-dimensional pp-waves, Comm. Math. Phys. 296 no.3 (2010), 881-898. arXiv:0810.2903

[M10] B. Michel, Masse des opérateurs GJMS. arXiv:1012.4414 
[P08] S. Paneitz, A quartic conformally covariant differential operator for arbitrary pseudoRiemannian manifolds (summary), SIGMA Symmetry Integrability Geom. Methods Appl. 4 (2008), paper 036, 3p. arXiv:0803.4331

[W86] V. Wünsch, On conformally invariant differential operators, Mathematische Nachrichten 29 (1986), 269-281.

[W00] V. Wünsch, Some new conformal covariants, Journal for Analysis and its Applications 19 no.2 (2000), 339-357.

Humboldt-Universität, Institut für Mathematik, Unter Den Linden, D-10099 BerLin

E-mail address: ajuhl@math.hu-berlin.de

University Uppsala, Department of Mathematics, P.O. Box 480, S-75106 Uppsala

E-mail address: andreasj@math.uu.se 